\title{
Cardiovascular Molecular Imaging
}

\section{Inauguraldissertation}

\author{
zur \\ Erlangung der Würde eines Doktors der Philosophie \\ vorgelegt der \\ Philosophisch-Naturwissenschaftlichen Fakultät \\ der Universität Basel \\ von
}

\section{Elham Khanicheh}

\author{
aus Teheran, Iran
}

Basel, 2013

Originaldokument gespeichert auf dem Dokumentenserver der Universität Basel edoc.unibas.ch

Dieses Werk ist unter dem Vertrag „Creative Commons Namensnennung-Keine kommerzielle Nutzung-Keine Bearbeitung 2.5 Schweiz“ lizenziert. Die vollständige Lizenz kann unter creativecommons.org/licences/by-nc-nd/2.5/ch eingesehen werden. 
Genehmigt von der Philosophisch Naturwissenschaftlichen Fakultät auf Antrag von

Prof. Dr. Jörg. Huwyler

PD Dr. med. Beat A. Kaufmann

Prof. Dr. Christoph. Handschin

Basel, Den 23.04.13

Prof. Dr. Jörg Schibler 


\section{C.Creative}

Namensnennung-Keine kommerzielle Nutzung-Keine Bearbeitung 2.5 Schweiz

Sie dürfen:

das Werk vervielfältigen, verbreiten und öffentlich zugänglich machen

\section{Zu den folgenden Bedingungen:}

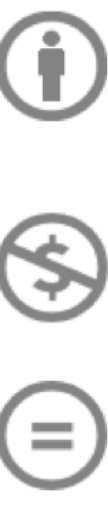

Namensnennung. Sie müssen den Namen des Autors/Rechteinhabers in der von inm festgelegten Weise nennen (wodurch aber nicht der Eindruck entstehen darf, Sie oder die Nutzung des Werkes durch Sie würden entlohnt).

Keine kommerzielle Nutzung. Dieses Werk darf nicht für kommerzielle Zwecke verwendet werden.

Keine Bearbeitung. Dieses Werk darf nicht bearbeitet oder in anderer Weise verändert werden.

- Im Falle einer Verbreitung müssen Sie anderen die Lizenzbedingungen, unter welche dieses Werk fällt, mitteilen. Am Einfachsten ist es, einen Link auf diese Seite einzubinden.

- Jede der vorgenannten Bedingungen kann aufgehoben werden, sofern Sie die Einwilligung des Rechteinhabers dazu erhalten.

- Diese Lizenz lässt die Urheberpersönlichkeitsrechte unberührt.

\section{Die gesetzlichen Schranken des Urheberrechts bleiben hiervon unberührt.}

Die Commons Deed ist eine Zusammenfassung des Lizenzvertrags in allgemeinverständlicher Sprache: http://creativecommons.org/licenses/by-nc-nd/2.5/ch/legalcode.de

Haftungsausschluss:

Die Commons Deed ist kein Lizenzvertrag. Sie ist lediglich ein Referenztext, der den zugrundeliegenden Lizenzvertrag übersichtlich und in allgemeinverständlicher Sprache wiedergibt. Die Deed selbst entfaltet keine juristische Wirkung und erscheint im eigentlichen Lizenzvertrag nicht. Creative Commons ist keine Rechtsanwaltsgesellschaft und leistet keine Rechtsberatung. Die Weitergabe und Verlinkung des Commons Deeds führt zu keinem Mandatsverhältnis. 


\section{Acknowledgments}

First and foremost I would like to express my deep gratitude and appreciation to my supervisor PD. Dr. med Beat A. Kaufmann for giving me the opportunity to do my $\mathrm{PhD}$ in his research group, for introducing me to the exciting field of molecular imaging and for teaching me the relevant skills from the basics to the more complicated techniques. During this joyful journey his valuable inputs, constructive discussions and his endless support helped me to grow and to develop the means to become a clinician-researcher. I would like to thank him not only because he supervised me and thought me several skills in the past three years at the lab but also because he kindly supported me to plan my future career.

I am very grateful to Prof. Dr. Huwyler for being my faculty representative at the faculty of natural sciences and to Prof. Dr. Handschin for co-reporting my thesis.

Furthermore, I would like to thank PD. Dr. med Gabriela M. Kuster and her group for their kind collaboration and their valuable inputs.

Many thanks to Martina Mitterhuber and Lifen Xu from the Cardiovascular Molecular Imaging laboratory for their scientific feedbacks and their technical support and also for all the non-serious pleasant chats. I am also grateful to my other friends who cheered me up and helped me to keep my spirit up during my PhD studies.

And last but most importantly I am very thankful to my parents and my sisters Azadeh and Arezu. Thank you very much for believing in me, for supporting me and encouraging me to follow my dreams. 


\section{Table of Contents}

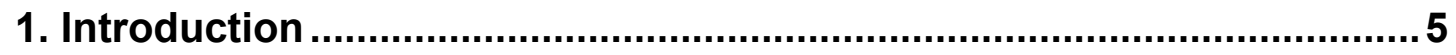

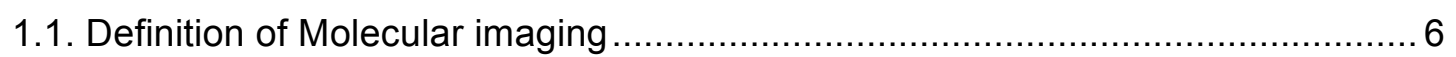

1.2 Contrast enhanced ultrasound molecular imaging ......................................... 8

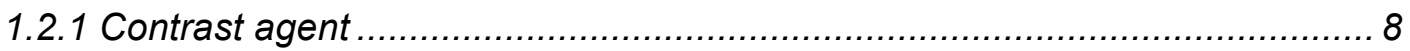

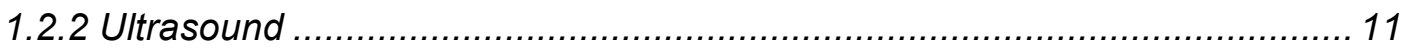

1.2.3 Contrast specific ultrasound imaging...................................................... 16

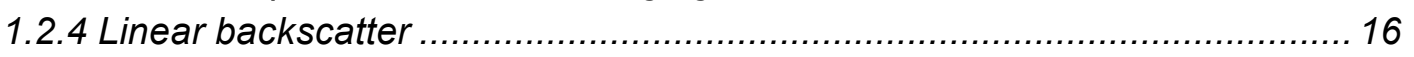

1.2.5 Non-linear backscatter: Harmonic imaging ............................................ 17

1.2.6 Non- linear backscatter: Pulse inversion imaging....................................... 18

1.2.7 Non-linear backscatter: Power modulation ............................................... 20

1.3 Targeting of contrast agent for moelcular imaging........................................... 21

1.4 Targeting and molecular imaging in large arteries ............................................ 24

1.5 Detection of targeted microbubbles: Imaging strategies...................................25

1.6 Advantages and disadvantages of ultrasound based- imaging ........................27

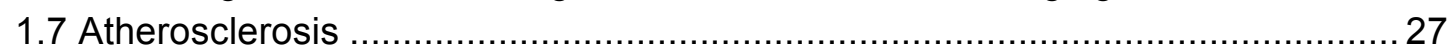

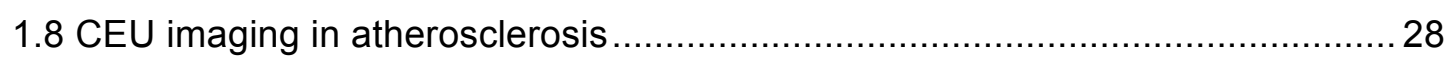

2. Aims of the PhD project .................................................................... 31

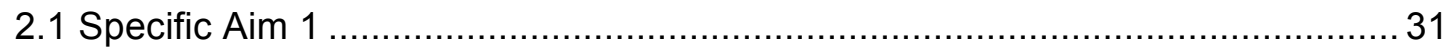

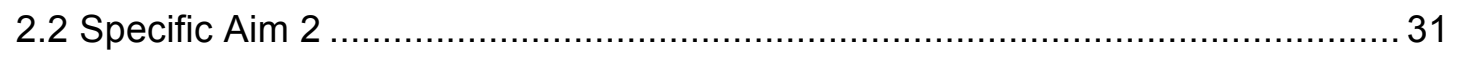

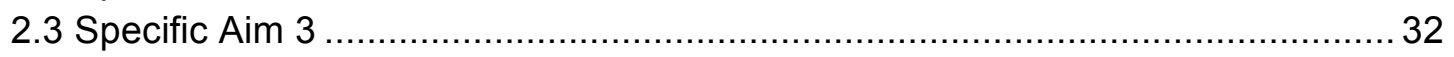

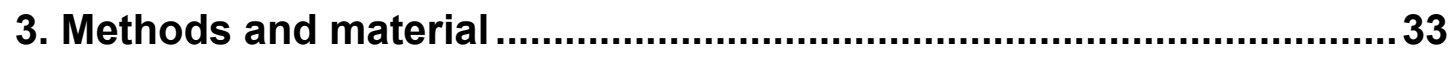

3.1 Factors affecting the endothelial retention of targeted microbbles: influence of mirobubble shell design and cell surface projection of the endothelial target molecule.

3.2 Noninvasive ultrasound molecular imaging of the effects of Statins on endothelial inflammatory phenotype in early atherosclerosis................................................. 41

3.3 Rapid reduction of endothelial activation in early- stage atherosclerosis with apocynin independent of anti- oxidative properties.................................................. 51

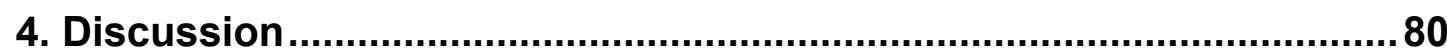

4.1 The influence of the length of functionalized polyethylene glycol spacer arms on improving the targeting efficiency of microbubbles ............................................... 80 4.2 noninvasive ultrasound molecular imaging of the effect of statins on endothelial inflammatory phenotype in early murine atherosclerosis.

4.3 The short-term effects of treatment with apocynin on endothelial inflammation application of ultrasound molecular imaging

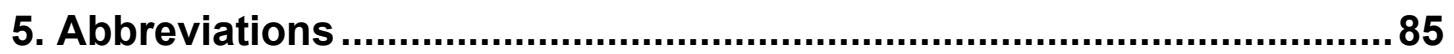

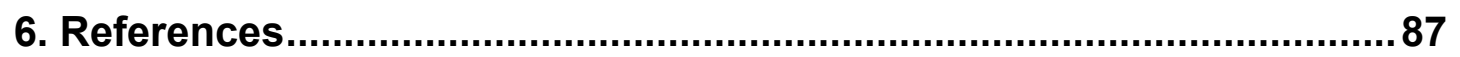

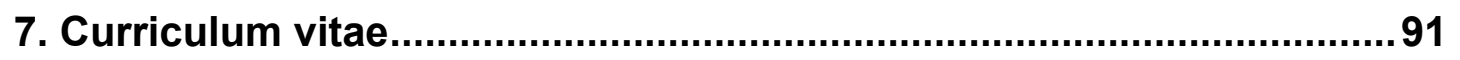




\section{Introduction}

Although there have been significant improvements in the treatment of cardiovascular diseases they still remain the main cause of morbidity and mortality globally. Increased burden of cardiovascular diseases on both individuals and societies has led governments and health care professionals to invest more avidly on preventive measurements. Primary prevention includes early diagnosis and treatment of the disease and risk stratifications before diseases are in advanced stages and thus require costly and often invasive therapeutic approaches.

Traditionally, in cardiovascular medicine, the diagnosis, monitoring of the progression of disease and the evaluation of therapeutic effects are accomplished by studying the anatomy and the physiological consequences manifested as changes in flow, metabolism and function of the heart and/or blood vessels. However, in early disease stages these approaches may not be adequate to detect pathologic changes, therefore the ability to image noninvasively pathophysiologic processes on a molecular level is thought to provide an opportunity in the future to enhance the diagnostic capabilities. Techniques for molecular imaging have been developed for essentially all forms of medical imaging. These techniques generally involve the formulation of novel site-targeted contrast agents with the goal of detecting the molecular footprint specific for a particular disease state.

The application of molecular imaging technologies is currently in preclinical research. Translation of these approaches to the clinical field may provide additional information on the molecular and pathophysiological process of disease and possibly a mean to personalize approaches for the evaluation and management of disease. 


\subsection{Definition of Molecular imaging}

Molecular imaging can be defined as the visualization, characterization and noninvasive measurement of a biological process at a molecular and cellular level. Molecular imaging technologies have been developed over the past two decades, initially using nuclear imaging techniques. Subsequently, molecular imaging technologies have been developed for all other imaging methods available including computed tomography (CT) imaging, magnetic resonance (MR) imaging, optical fluorescence and bioluminescence imaging and also for ultrasound based imaging. All these methods have in common that they use a contrast agent that is targeted to a specific molecule present in the tissue. The use of a contrast agent for molecular imaging requires interaction and retention of the agent with an endogenous molecule (1). Potential clinical and research applications of molecular imaging methods are summarized in figure $1(2)$.

\section{Clinical Applications}

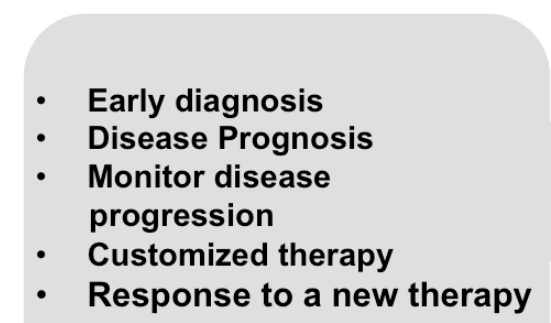

Research Applications

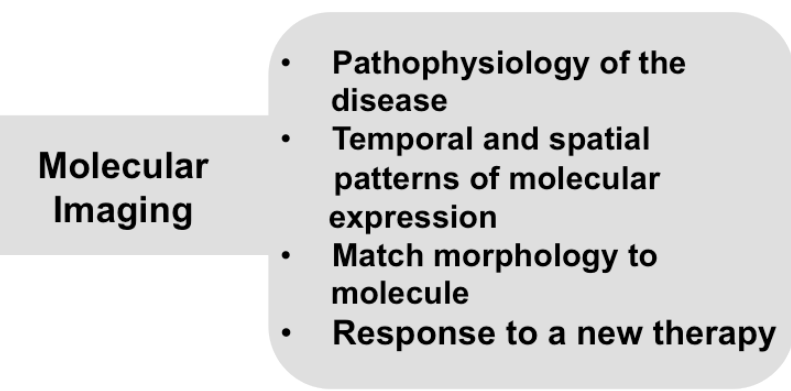

Figure1. Molecular imaging can be used for clinical, research purposes or both. Adapted from Inaba Y, Lindner JR. Molecular imaging of disease with targeted contrast ultrasound imaging. TransI Res. 2012 Mar; 159(3):140-8. (2)

Although molecular imaging has been developed for almost all forms of medical imaging modalities, there are significant differences in terms of spatial resolution, sensitivity and practicability between different detection methods (ultrasound, gamma radiation detector, magnetic resonance, light 
and ect.). Differences in the properties of the imaging probes additionally influence relative toxicity, sensitivity, specificity, biodistribution and temporal resolution of the images. Table 1 presents selected operational parameters for different imaging modalities. High sensitivity, availability, relatively low cost and rapid imaging execution protocols are properties of molecular imaging with ultrasound detectors that make ultrasound molecular imaging particularly interesting for screening large numbers of patients for potential diseases including cardiovascular disorders (3).

Table 1. Comparison of molecular imaging technologies. Adapted from Sinusas AJ et al. Multimodality cardiovascular molecular imaging, Part I. Circ Cardiovasc Imaging. 2008 Nov; 1(3):244-56(1). PET represents positron emission tomography and SPECT, single photon emission computed tomography.

\begin{tabular}{|l|l|l|l|l|}
\hline Imaging modality & $\begin{array}{l}\text { Spatial } \\
\text { resolution }\end{array}$ & $\begin{array}{l}\text { Depth of } \\
\text { penetration }\end{array}$ & $\begin{array}{l}\text { Temporal } \\
\text { resolution }\end{array}$ & $\begin{array}{l}\text { Sensitivity } \\
\text { (mol/L) }\end{array}$ \\
\hline PET & $1-2 \mathrm{~mm}$ & No limit & Sec-min & $10^{-11}-10^{-12}$ \\
\hline SPECT & $0.3-1 \mathrm{~mm}$ & No limit & min & $10^{-10}-10^{-11}$ \\
\hline MRI & $50-250 \mu \mathrm{m}$ & No limit & Min-hrs & $10^{13}-10^{-5}$ \\
\hline X-ray CT & $25-150 \mu \mathrm{m}$ & No limit & min & No data \\
\hline Ultrasound & $30-500 \mu \mathrm{m}$ & mm-cm & Sec-min & $10^{-6}-10^{-9}$ \\
\hline
\end{tabular}




\subsection{Contrast enhanced ultrasound molecular imaging}

A wide spectrum of non- invasive molecular imaging techniques to evaluate the phenotype of diseased tissue in vivo has been developed. The use of these methods in research and in the clinical field may overcome the shortcomings of traditional imaging modalities, which evaluate anatomy or physiology of the organs. The application of these techniques in cardiovascular diseases may improve our understanding of pathophysiology of the disease, diagnose life-threatening disease at early stages, monitor disease progression, assess response to novel and established therapies and finally select appropriate therapies according to the phenotype of the disease. Contrast Enhanced Ultrasound (CEU) molecular imaging is among these newly developed imaging modalities that have been of interest to both clinicians and researchers. CEU molecular imaging relies on selective targeting and retention of imaging probes at the site of disease. So to better understand this imaging technique it is worthwhile to discuss 1) the properties of contrast agents and for the ultrasound imaging 2) the physical properties of ultrasound waves and 3) contrast-specific ultrasound imaging protocols.

\subsubsection{Contrast agent}

The main requirements for ultrasound contrast agents are that they should be easy to administer to the circulatory system, be stable enough throughout the duration of image acquisition, have low toxicity and finally provide the highest possible contrast-to-tissue ratio of acoustic reflection. The use of gas bubbles to enhance the blood pool for ultrasound imaging goes back to 1968 when Gramiak and Shah during echocardiography injected agitated normal saline into the ascending aorta. They could detect strong echoes in the aorta and the heart chambers. They concluded that these strong echoes were due to the air bubbles, formed by agitation or cavitation during injection(4). In 1984 
Feinstein et al produced albumin encapsulated microbubbles by sonication of human serum albumin and showed that these could be visualized in the left ventricle after intravenous injection (5).

Since then, ultrasound contrast agents have been developed and approved by regulatory authorities for various clinical applications such as left ventricle cavity opacification (Figure 2) or characterization of liver masses by assessing vascular filling patterns. These contrast agents are gas-filled microbubbles, which are smaller than red blood cells (RBC) and therefore are able to circulate freely in the vasculature, and thus are called 'blood pool' agents. Contrast agents act by either their presence in the circulation ('blood pool' agents) or by their selective retention or uptake by cells following a vascular phase(6).
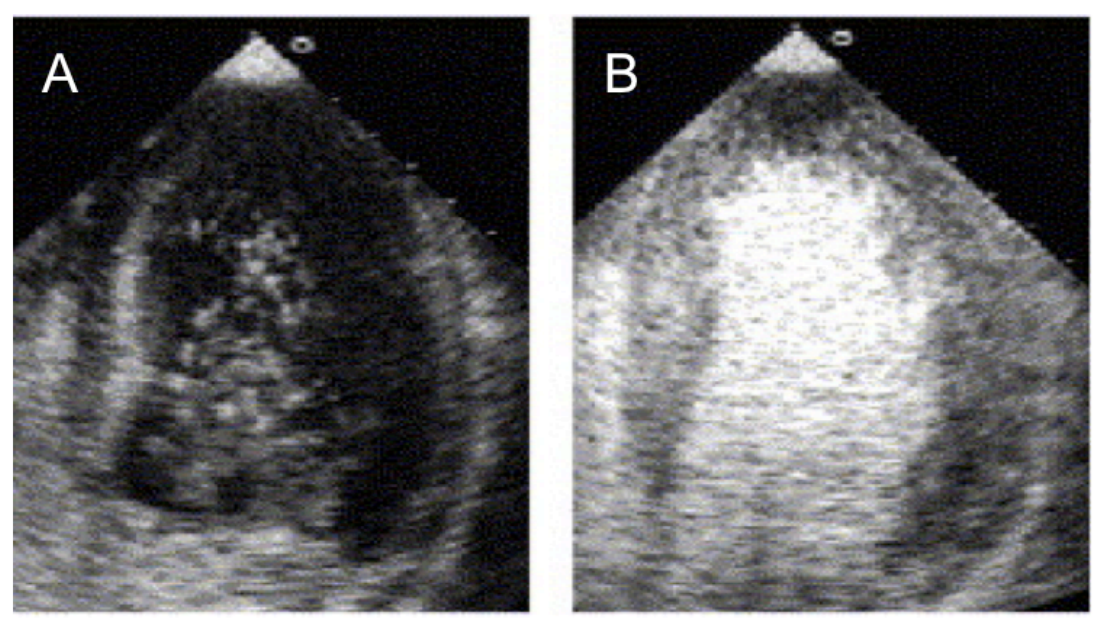

Figure 2. Examples of apical four-chamber ultrasound plane images obtained from a patient before (A) and after injection of contrast agent (B). At the pre-contrast image delineation of myocardium and left ventricular cavity is not clear $(A)$ while after the injection of contrast agent the contrast between myocardium and left ventricular cavity has been increased (B). 
Microbubbles are composed of a shell and a gas core. The shell is composed of albumin, lipids or biocompatible polymers. The shell increases the stability of the contrast agents by decreasing the gas volume loss, reducing surface tension and additionally controls the size distribution during the production process. The microbubble shell is thin and measure between 3 nanometers in lipid-encapsulated and 20 nanometers in albumin-encapsulated agents and therefore eventually will allow a diffusible gas such as air to leak out and return to the blood resulting in a relatively short half life of air-filled microbubbles. Therefore instead of air many manufacturers take advantage of gases with a high molecular weight and low solubility properties (perflurocarbones, sulfur-hexafluroride). The low diffusion rate results in an increased half-life of microbubbles in the circulation (6).

Ultrasound contrast agents currently in use vary in size from several hundred nanometers to a few micrometers in diameter. The size of the acoustic particles determines their stability in the circulation, their passage through the pulmonary capillary network necessary for reaching the systemic circulation, and their acoustic properties. For clinical use contrast agents are

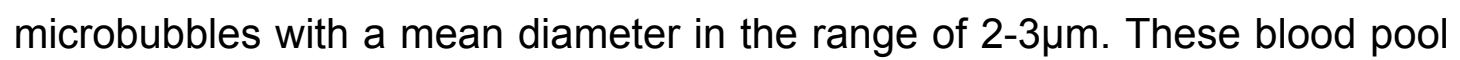
agents are characterized by flow dynamics similar to red blood cells and eventually are taken up by the reticuloendothelial system where they are metabolized by liver Kupffer cells (6).

Lipid shelled microbubbles have several specific properties that make them interesting for clinicians and researchers. Phospholipids spontaneously assemble in monolayers at gas-water interfaces. Phospholipids are ideal agents for the microbubble shell as they can oscillate, that is to say expand and compress during insonification. Finally, lipid shells of microbubbles can be easily functionalized for molecular imaging or drug delivery by incorporating various lipid head group species into the shell that can be used for the attachment of targeting ligands (7).

To increase the longevity of lipid shelled microbubbles in circulation, polyethylenglycol (PEG) compounds are also incorporated into the 
microbubble shell. The exact physical basis of the increased half life of PEG microbubbles in vivo as well as the mechanism of interaction of the PEG microbubbles with cells is not yet fully understood. It has been shown that in the presence of a PEG brush on the surface of microbubbles, hydrostatic and electrostatic interactions between microbubbles and plasma proteins such as complement components will decrease $(8,9)$. Decreased deposition of complement on the surface of PEG microbubbles in turn decreases the uptake of mircobbbles by the reticuloendothelial system and might be responsible for the longer half life of microbubbles $(10,11)$. However recently it has been demonstrated that PEG does little to inhibit deposition of complement on microbubble surface but provides a steric barrier to interaction of complement deposited on microbubbles with endothelial cell surface receptors $(12,13)$.

\subsubsection{Ultrasound}

Ultrasound waves are sound waves with higher frequencies than audible sound. Audible sound wave frequencies range from 20 hertz $(\mathrm{Hz})$ to 20,000 $\mathrm{Hz}(20 \mathrm{kHz})$. Any sound wave with frequencies above $20 \mathrm{KHz}$ is not audible for the human ear and is referred to as ultrasound. In medical imaging, alternating electricity-induced deformation of a piezoelectric element is used to generate ultrasound waves. The piezoelectric element not only transmits the ultrasound wave but also receives returned echoes reflected by the tissue interfaces. In clinical imaging applications the frequencies in use range between $2-15 \mathrm{MHz}$ although frequencies up to $40 \mathrm{MHz}$ may also be used for special applications.

A sound wave is a longitudinal wave consisting of cyclic pressure variations. As sound travels it causes the particles in its path to be displaced in the direction of the wave propagation. The speed of the propagation of sound is 
determined by the stiffness and density of a media it is travelling in. The ultrasound wavelength is defined as the distance of two identical consecutive

points on a sinusoidal curve describing the cyclic pressure variations of the ultrasound wave. The wavelength can be calculated by dividing the speed of sound in a specific media by the original frequency of the source of sound (Figure 3). The wavelength of the ultrasound determines the image resolution, a shorter wavelength results in a higher image resolution.

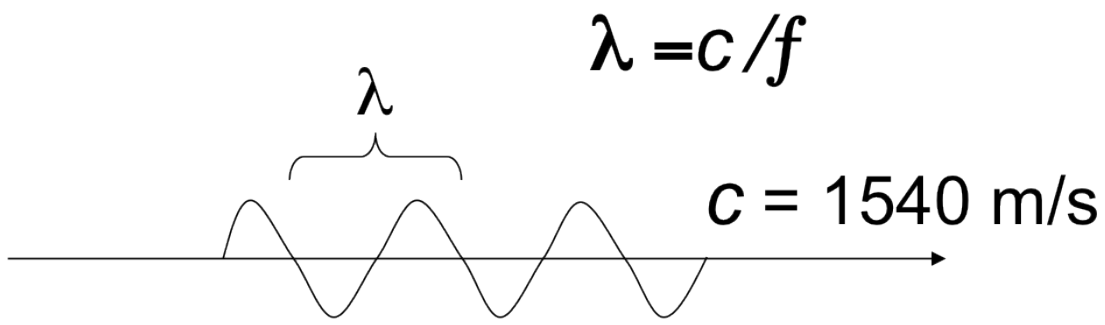

Figure 3. The wavelength of a wave is the distance of two identical consecutive points on the wave. It is obtained by dividing the average sound velocity in soft tissue, which is $1540 \mathrm{~m} / \mathrm{s}$ by the frequency of the source of ultrasound.

A specific property of an ultrasound wave, as opposed to an audible sound wave, is that it can be sent from the ultrasound source into a specific direction. The amplitude of the sound wave is represented by the maximum pressure variations above and below the baseline measured in pressure units (megapascals; MPa)(Figure 4). The amount of energy that can be transferred to tissue per unit of time is defined as the power of the ultrasound beam. The power per cross section area of the beam reflects the intensity of the beam. The intensity increases when 1) the power increases or 2) when there is a decrease in the cross section area of the beam by adjusting the focus of the ultrasound beam. The estimated peak intensity at the focal point of the ultrasound beam is represented by the Mechanical Index (MI) derived from the average peak negative pressure of the beam $(P)$ divided by the square root of the transmitted frequency $(f)$ :

$$
\mathrm{Ml}=\mathrm{P} / \sqrt{ } \mathrm{f}
$$


As ultrasound travels through the tissue the intensity and amplitude of the beam decreases, a phenomenon named attenuation, which is measured in $\mathrm{dB}$. Attenuation occurs predominantly due to the absorption of the ultrasound beam in tissue. Attenuation depends on the distance travelled and also the frequency of ultrasound, where attenuation is larger for higher frequencies.

Peak positive acoustic pressure(MPa)

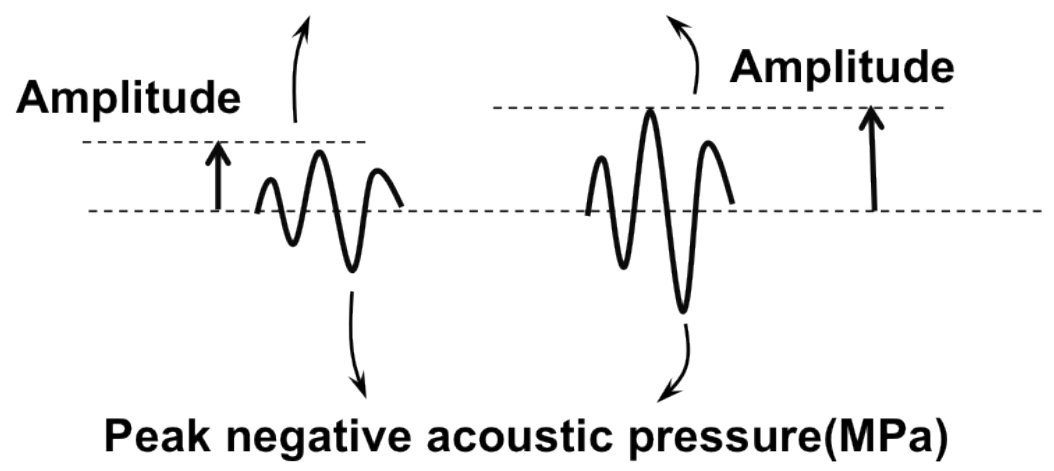

Figure 4. Amplitude of the sound wave is represented by the maximum pressure variations above and below the baseline.

In soft tissue the average propagation speed of ultrasound is $1540 \mathrm{~m} / \mathrm{s}$. It is higher in less compressible media such as bone and lower in more compressible media such as air. The propagation speed is used to determine the distance of a structure from the source of the wave and confines it in an appropriate location on the formed image (Figure 5). 

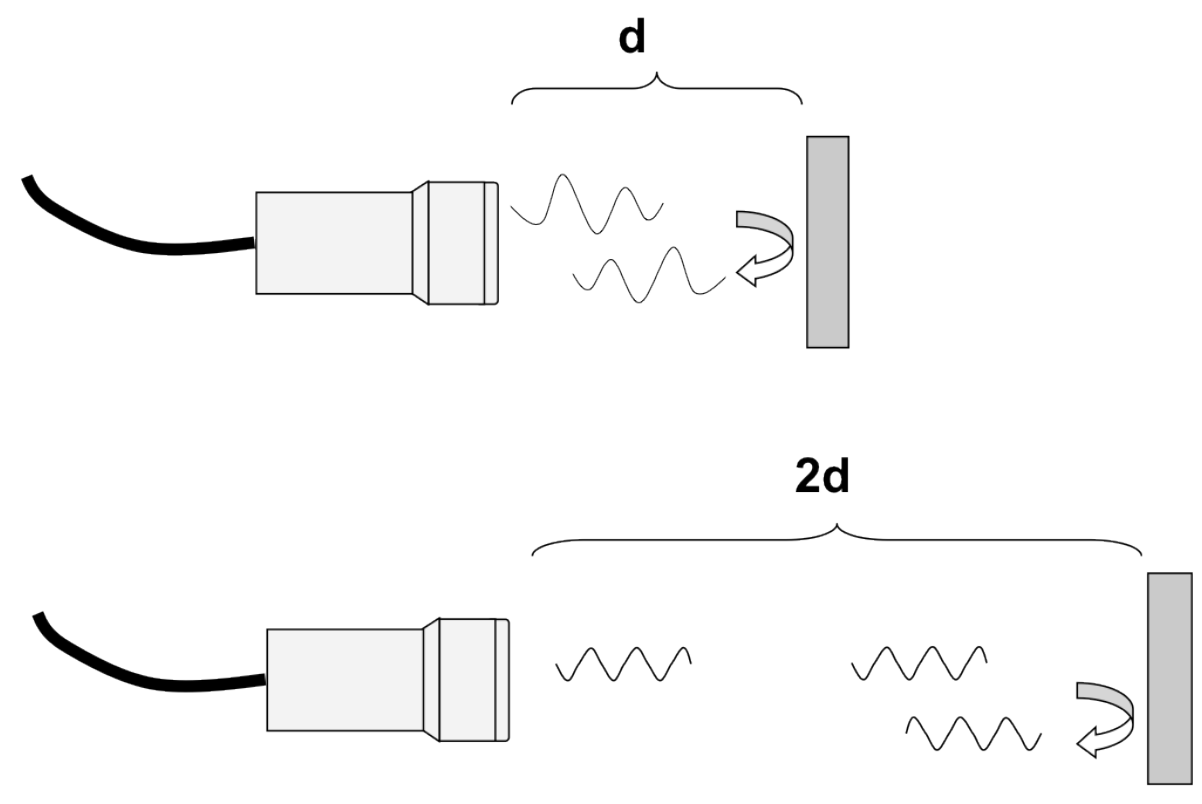

Figure 5. The propagation speed and the time that is required for a sound wave to return to its source are used to determine the distance of a structure from the source of wave.

While the ultrasound wave is travelling through the tissue it encounters different interfaces with different acoustic properties, which will influence the propagation. Propagation also depends on the angle of incidence $\left(\theta_{i}\right)$ of the beam to the interface. When encountering an interface between two structures with different acoustic properties, a part of the ultrasound wave is reflected and returns toward the source of sound and the reminder is refracted. The proportion of reflected or scattered sound waves that travels back to the source of sound (scan head) is used to generate the image of the examined structure (Figure 6). 


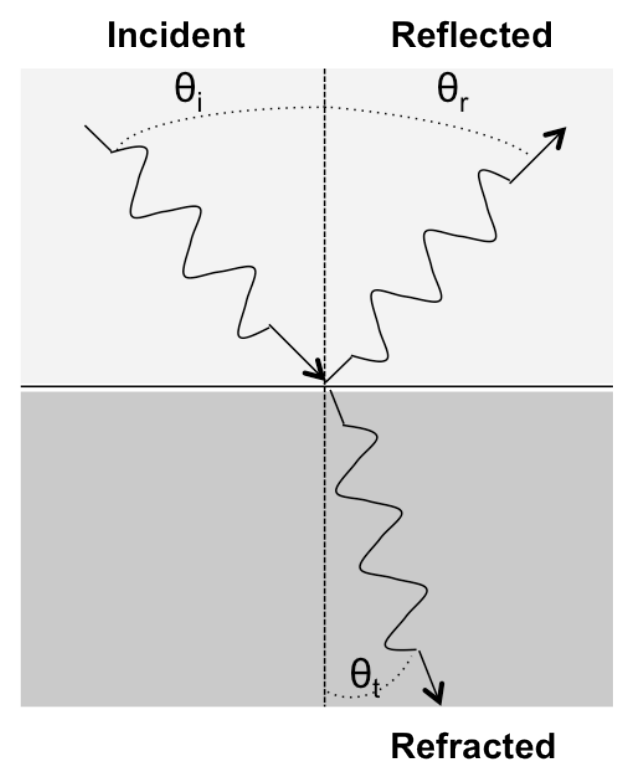

Figure 6. Facing an interface ultrasound is partially reflected and returns toward the source of sound and the rest is refracted.

Eventually as ultrasound is a compression wave (MI dependent mechanical effects) and is absorbed by the tissue (Thermal index heating effect) it can interact with the tissue. Ultrasound at high intensity doses can cause immediate thermal and mechanical effects at the time of exposure. However the long term effects of high intensity ultrasound on humans is not yet clear. Therefore the safety of using ultrasound in medical imaging is of consideration. According to international safety requirements the $\mathrm{MI}$ and $\mathrm{TI}$ of the ultrasound should be displayed on the ultrasound systems and in the case of requiring $\mathrm{Ml}$ or $\mathrm{TI}$ above curtain levels benefits from ultrasound examinations should be weighed against its risks. 


\subsubsection{Contrast specific ultrasound imaging}

When microbubbles are exposed to an ultrasound field they expand during the peak negative portion of the ultrasound beam and were compressed during positive peak portion. The ideal resonant frequency of microbubbles is close to frequencies that are used for clinical imaging. Therefore when microbubbles are exposed to the ultrasound beam they resonate efficiently. Unlike tissue, when microbubbles are exposed to various amplitude sounds they behave in different ways. At lower incident pressures bubbles produce linear backscatter, which can be used to enhance echo from blood. At higher incident pressures beyond 50-100 kPa backscatters from bubbles show nonlinear characteristics. These properties of bubbles were used to develop contrast specific imaging protocols such as harmonic and pulse inversion imaging modes (see below). When the incident pressure exceeds about 1 $\mathrm{MPa}$, bubbles for a short time emit non-linear backscatter and soon after will be destroyed.

\subsubsection{Linear backscatter}

Although bubbles are smaller than red blood cells and their concentration in blood after intravenous injection is small when compared to red blood cells, the amplitude of the backscattered echo from microbubbles is several orders of magnitude larger than backscattered signal from red blood cells. This can be explained by the mismatch in acoustic impedance (which itself is influenced by the density and compressibility of the object) between the bubble gas core and blood, and the efficient resonance properties of microbubbles when exposed to ultrasound frequencies used in clinical equipment. This mismatch in acoustic impedance permits signal enhancement in ultrasound imaging.

Thus, in fundamental frequency gray scale imaging, signal enhancement from microbubble contrast agents can be detected in the lumina of the ventricles or large vessels where the concentration of bubbles is high enough. However, in 
the case of the imaging of smaller myocardial vessels the contrast is not normally detectable due to high echogenicity of the myocardium itself. Thus, in order to further increase the contrast-to-tissue signal difference, microbubble-specific imaging techniques have been developed.

\subsubsection{Non-linear backscatter: Harmonic imaging}

As microbubbles in the ultrasound beam are subjected to low-pressures (less than $100 \mathrm{kPa}$ or $\mathrm{Ml}$ of less than 0.1 ) they start to resonate and undergo compression and rarefaction phases around their radius in harmony with the cyclic pressure variations of the ultrasound wave. Ultrasound signal emitted by bubbles at this pressure is received by the transducer at the same frequency as the incident beam. Such frequencies are called fundamental frequency. If microbubbles are driven by a sufficiently large acoustic pressure (more than $100 \mathrm{kPa}$ or $\mathrm{Ml}$ of more than 0.1 ) they reach a point where the cyclic expansion and compression does not occur linearly anymore, but rather asymmetric. Thus the frequencies of the emitted echoes from bubbles are not the same as incident frequencies but exact multiples of the fundamental frequency. These frequencies are named harmonic frequencies (6). This non- linear response of microbubbles in the ultrasound beam can be used to distinguish the signal of contrast agent from surrounding tissue (Figure7). 


\section{Echo at fundamental \\ frequency $\left(f_{0}\right)$}

Tissue

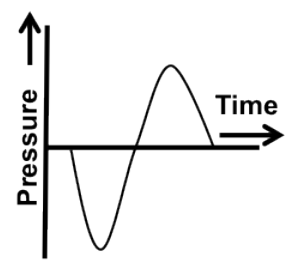

Microbubble

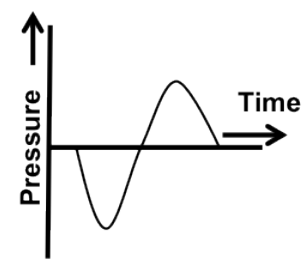

Echo at second harmonic

frequency $\left(2 f_{0}\right)$
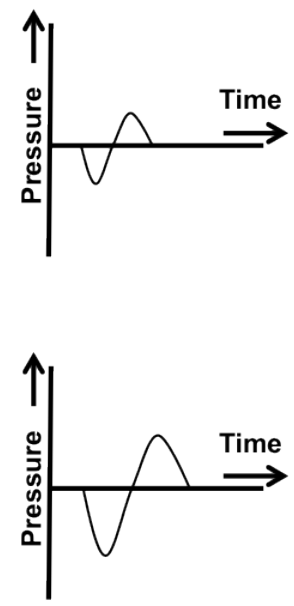

Figure 7. Schematic illustration of the improved ratio of signal from microbubbles to the signal from tissue when receiving echoes at the second harmonic frequency $\left(2 f_{0}\right)$ rather than the fundamental frequencies $\left(f_{0}\right)$.

To increase the signal from contrast agent to the noise from tissue one strategy would be subtraction of background acquired from precontrast frames. The resulting frame reflects only the signal from contrast agent (14).

\subsubsection{Non- linear backscatter: Pulse inversion imaging}

In harmonic imaging, the transmitted frequencies should be set close to the band around fundamental frequencies while received frequencies should be restricted to the second harmonic frequencies of contrast agent. This setting limits image resolution from contrast agent because of restricted bandwidth. Therefore to overcome the conflict between the requirements of contrast detection and resolution in harmonic imaging and to surpass the signal from tissue, pulse inversion protocols have been developed and implemented on ultrasound scanners.

This method also relies on non-linear oscillation of bubbles in the ultrasound field. However it detects all nonlinear components of echoed sound including 
second harmonics over the entire bandwidth of the transducer. In this method two successive sound pulses are transmitted into the tissue. The second pulse is a mirror image of the first pulse. The transducer receives the echoes of these pulses and sums them up. If the ultrasound pulses are reflected from tissue, due to the linear behavior of tissue the sum of the pulses will cancel out. However, microbubbles will behave non-linearly in response to the same ultrasound pulses which will be changed and backscattered asymmetrically and therefore when summed will not equal zero. Thus the signal from microbubbles will be detected by the scanner but not the signal from tissue (Figure 8). One advantage of pulse inversion protocol over harmonic imaging is that the transducer receives a complete bandwidth of the reflected ultrasound. This allows forming a high-resolution image from bubbles in real time (15).

1. First pulse

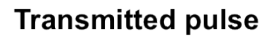

Tissue Echo
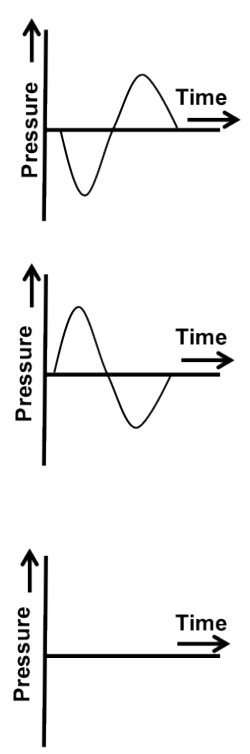

Bubble Echo
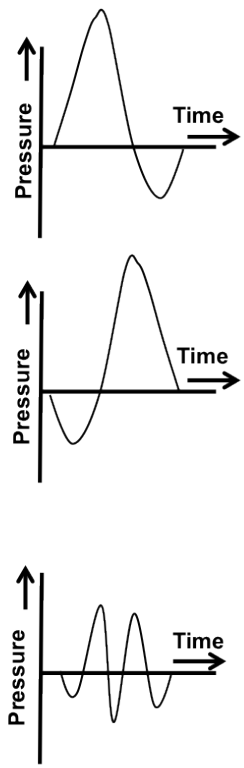

Figure 8. Schematic illustrations depicting methods used to improve microbubble signal relative to tissue with a pulse inversion imaging protocol. In this method, two or more sequential pulses with an inverted phase are transmitted along the same line. The sum of echoed pulses from the tissue will result in eliminating the signal from the tissue while due to the non-linear behavior of the microbubbles the signal will not be eliminated. (Adapted from Harald Becher, Peter N. Burns, chapter one, Handbook of Contrast Echocardiography, springer,2000) 


\subsubsection{Non-linear backscatter: Power modulation}

At low power imaging, signal to noise ratio could also be increased by using a power modulation technique. In this method instead of using two pulses with different acoustic phases, two pulses with different acoustic power or amplitude are used. A first pulse would be transmitted at low power and the second at very low power, at half the amplitude of the first pulse. During image processing the echoes from the second pulse are doubled in amplitude and subtracted from the first pulse. Therefore, the echoes from tissue will be canceled due to linear behavior of tissue under low power ultrasound waves. In contrast, the signal from bubbles will not be nullified as bubbles behave nonlinearly in low power pulses but not at very low power pulses (Figure 9).

Tissue Echo(Linear)

A
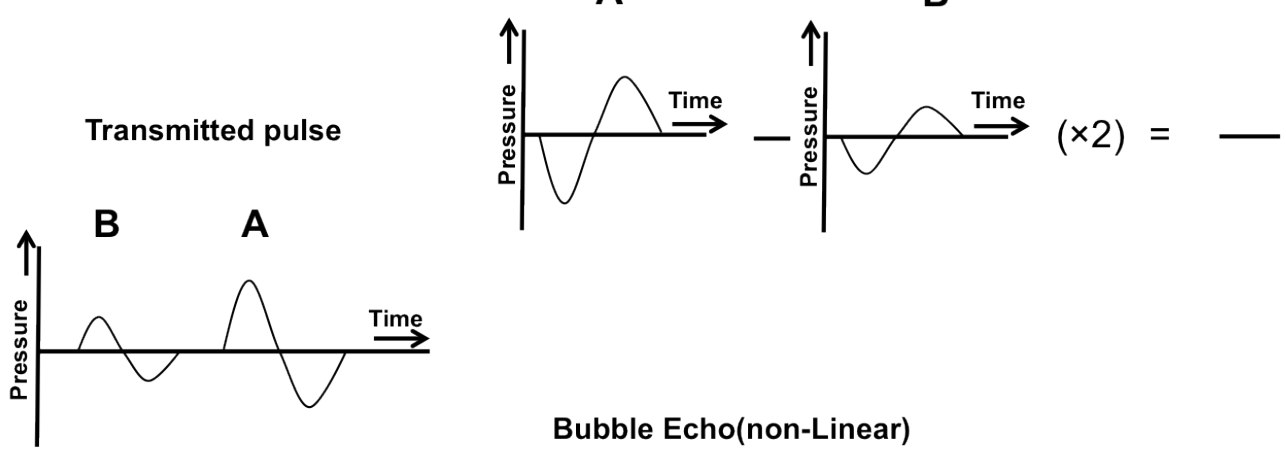

Bubble Echo(non-Linear)

A

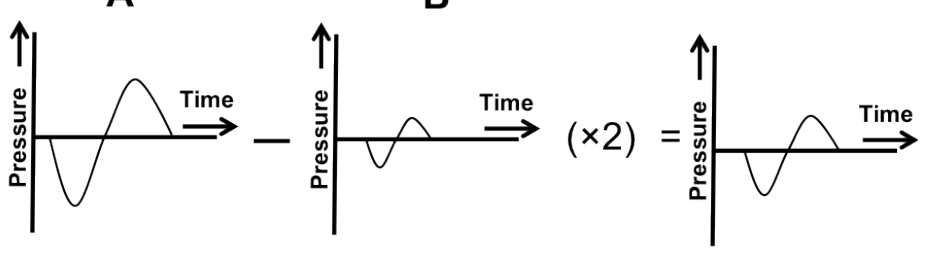

Figure 9. Schematic illustration depicting the method used to improve microbubble signal relative to tissue with a power modulation imaging protocol. In this protocol two consecutive pulses one with the full- amplitude and the other with the half-amplitude are transmitted. The echoed signal from the half- amplitude pulse will be multiplied by two and subtracted from the full- amplitude echo. This protocol will result in eliminating the signal from the tissue while due to the non- linear behavior of the micribubbles the signal either at the fundamental or at the harmonic frequencies will not. 


\subsection{Targeting of contrast agent for moelcular imaging}

Molecular imaging with contrast- enhanced ultrasound relies on selective retention of targeted microbubbles at the site of disease. It is important to note that contrast agents, whether they are microbubbles or larger-sized nanoparticles, are entirely intravascular agents and they do not leave the vascular compartment $(16,17)$. Therefore to image pathophysiological processes microbubbles are targeted either to disease specific molecules expressed on the endothelial cells, blood cell components (activated leukocytes or platelets) or blood proteins such as fibrin(13,18-23). However, nanoparticles, because of their relatively smaller size and longer circulation times, in the regions where vascular permeability is disrupted would leak out and would be able to target extravascular components (24).

There are two general strategies to target contrast agents: 1) non- specific targeting and 2) specific targeting.

A simple approach for microbubble targeting is to take advantage of the innate properties of the shell of microbubbles to bind directly or indirectly to pathologically activated cells. This technique relies on the composition of albumin or lipid shell microbubbles $(17,25-28)$. For example incorporating anionic phospholipids into the lipid shell of the microbubbles has been shown to amplify complement-mediated attachment of microbubbles to activated leukocytes in regions of inflammation $(25,27,28)$ or to the complement receptors on the endothelium (13). This approach can be further modified by alternating the amount of steric hindrance from polyethylenglycol (PEG) in the shell composition (13). Albumin microbubbles additionally have the ability to bind to the $\beta 2$-integrins on activated leukocytes that recognize denatured albumin(25)

A more specific approach for microbubble targeting is the conjugation of target-specific ligands on the microbubble surface. This can be achieved either directly by covalent binding or indirectly by non-covalent links to the microbubble surface. The applied method depends on the nature of the microbubble surface (29). Functionalized polyethylenglycol spacer arms 
consisting of a phospholipid tail, which is incorporated into the lipid shell, a spacer part to project the ligand above the shell surface and an active chemical group are used for the conjugation process (figure 10) (30).

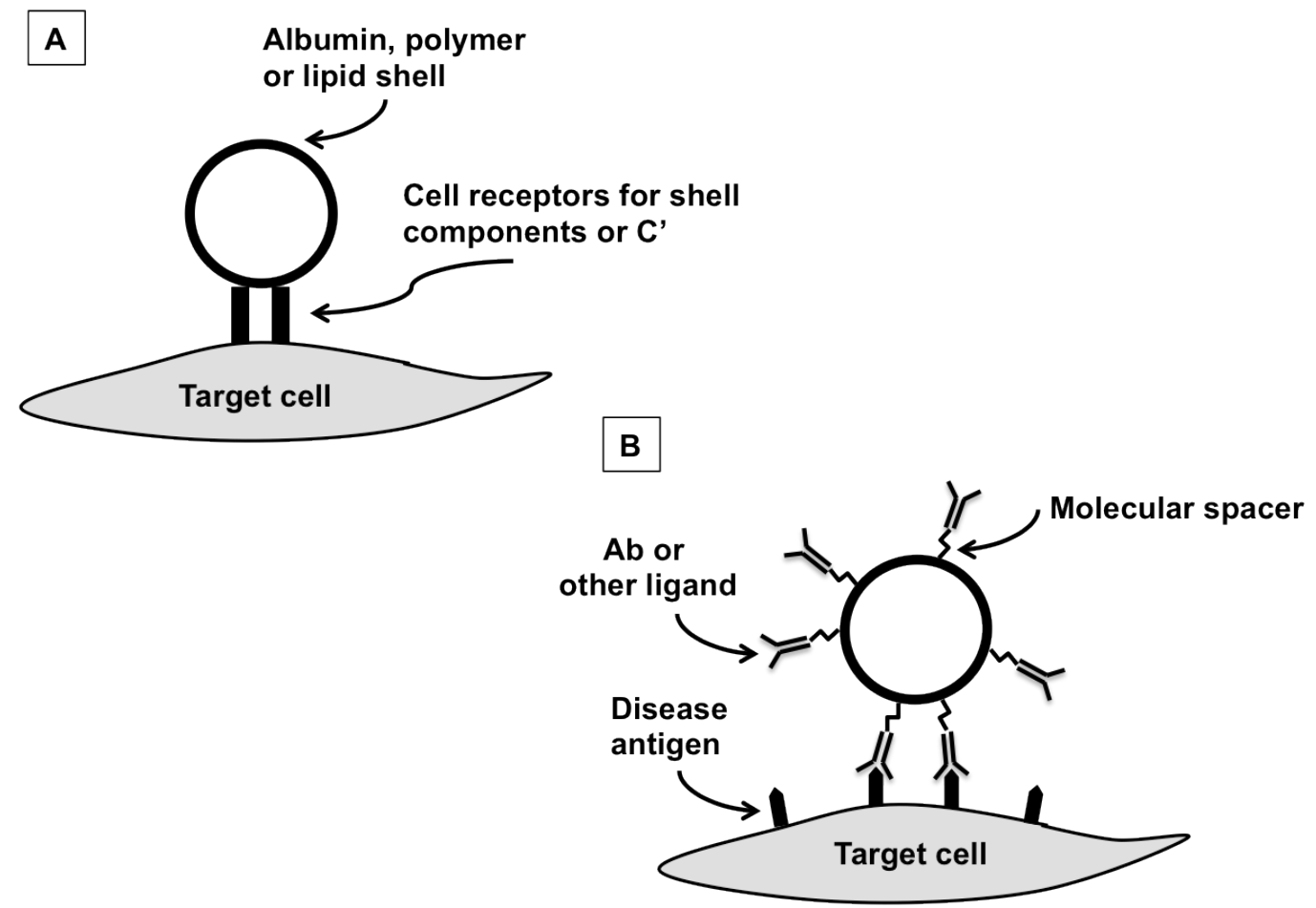

Figure 10. schematic illustration of strategies used for targeting contrast agents to disease specific antigens. Microbubbles are targeted non- specifically to a disease specific molecule or cell by modifying microbubble shell compositions (A) or specifically by conjugating a ligand specific for a disease molecule on their surface (B). Adapted from Piedra $\mathrm{M}$ et al. Molecular imaging with targeted contrast ultrasound. Cerebrovasc Dis. 2009;27 Suppl 2:66-74 (32).

Biotin-Avidin- biotin interaction is an example of a non-covalent bond, which is commonly used for targeting purposes of contrast agents in vitro and selected pre-clinical in vivo settings (Figure 11). Avidin has a high affinity for biotin and forms stable and strong bonds between biotinylated ligand and biotin- functionalized PEG spacer arms incorporated into the shell of the contrast agent under physiologic conditions (29). However, translation of this approach to clinical settings is limited due to the immunogenicity of avidin. 
Avidin is generally extracted from bacterial sources or white egg, which is a foreign protein and may have clinical limitations, particularly if it is used repeatedly. In addition avidin is a cationic macromolecule, which immediately forms in situ immunocomplexes when exposed to anionic sites within glomerular base membrane (31). Therefore, for clinical applications of ultrasound molecular imaging, other conjugation methods for targeting ligands will have to be established. For example small peptides can be covalently bound to PEG spacer arms functionalized by maleimide groups on the microbubble surface(29).

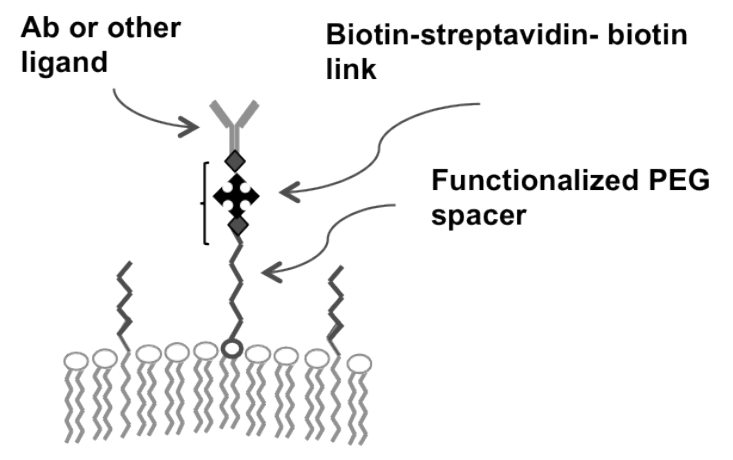

Figure 11. Example of non-covalent link strategy for contrast agent targeting. Functionalized PEG spacers bearing a biotin molecule at their tip are incorporated into the microbubble surface. Later a biotin- streptavidin- biotin linkage is used to conjugate the ligand to the microbubble shell.

Conjugation may be performed before or after preparation of the contrast agent depending on the properties of the ligand used and its tolerance to the chemical processing required for producing the contrast agent. Generally, several hundred to several thousand ligands per square micron of shell surface area can be conjugated to the contrast agent. 


\subsection{Targeting and molecular imaging in large arteries}

Several factors play a role for successful molecular imaging. The targeted molecule should be specific for the disease and should not be expressed constitutively on a large scale. As attachment of the contrast agent occurs under vascular flow, shear rate in the tissue of interest and bond affinity between the ligand on the contrast agent and the targeted molecule (on- and off-rate) are two factors with significant roles. In addition, ligand density on the contrast agent and site density of the targeted molecule are two other influencing factors that should be considered (Figure 12)(2,3).

As mentioned above, vascular shear rate is one of the factors influencing the extent of attachment of targeted microbubbles in arteries, where shear stress is high. CEU molecular imaging with antibody bearing targeted microbubbles has been successfully performed in the microvasculature of the myocardium, kidney and skeletal muscles where shear rate is low (around 3-5 dynes/cm2) (32-34). However, in large arteries such as the mouse aorta, shear rate is much higher with shear rate around 80 to 90 dynes $/ \mathrm{cm}^{2}(35,36)$. Given the low bond formation rate (low on rate) of antibody targeted microbubbles, successful attachment under high shear rate flows could be explained by the pulsatile nature of arterial flow. During diastole arterial flow rate is near zero thus bonds between targeted microbubbles and their endothelial target will form. These bonds are strong enough to endure high shear rates during systole. Flow chamber studies have demonstrated that under constant high flow the attachment of targeted microbubbles will dramatically decrease $(19,37)$. In contrast, simulations of pulsatile flow have illustrated that short interruptions of flow result in the attachment of targeted microbubbles. Resumption of flow did not cause detachment of microbubbles indicating the strength of the formed bonds (low off rate) (19). Regarding potential clinical applications, it should be mentioned that the wall shear rate in larger arteries in humans is lower compared to small animal models. Therefore attachment efficiency in humans should be better or similar to small animals (38). 


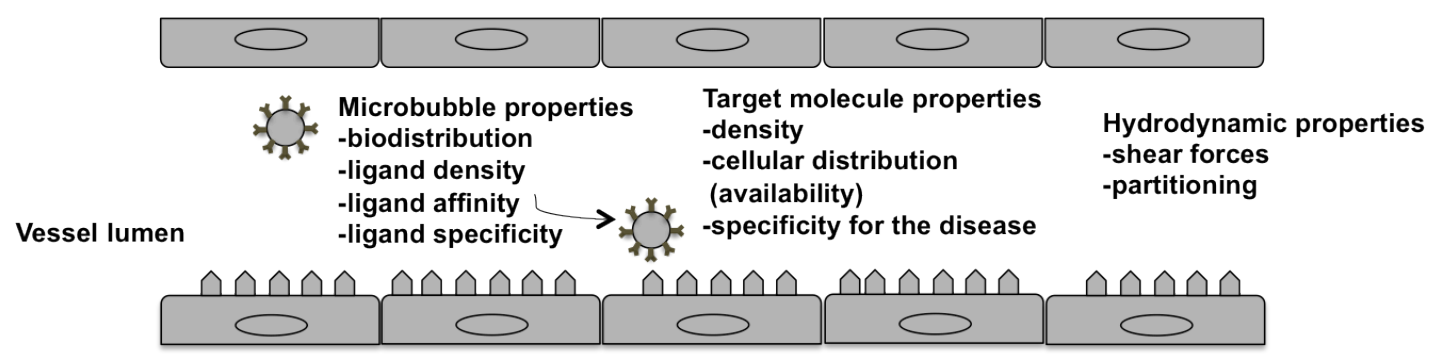

Figure 12) Determinants of targeting efficiency of ultrasound molecular imaging contrast agents. Targeting efficiency of microbubbles is influenced by the properties of the microbubbles and also targeted molecule as well as the hydrodynamics of vascular flow. Adapted from Beat $A$ Kaufmann et al. Molecular imaging with targeted contrast ultrasound. Current Opinion in Biotechnology 2007(3).

\subsection{Detection of targeted microbubbles: Imaging strategies}

Since generation of signal from microbubbles relies on their oscillation in the acoustic field it is important to know how this oscillation might be influenced by targeting of microbubbles and their subsequent attachment to the cells. There are two separate scenarios that should be considered. If targeted microbubbles are attached and phagocytized by activated leukocytes they are more stable when exposed to ultrasound energy and it is more difficult to destroy them. However they oscillate less efficiently and generate a smaller amplitude signal compared to freely circulating microbubbles $(27,39)$. On the other hand, it has been shown that attachment of targeted microbubbles to a cell surface such as endothelial cells, will improve stability of microubbles over time without damping the signal $(40,41)$.

To image attached targeted microbubbles with ultrasound in the tissue of interest, the strategy is to wait some minutes after the bolus injection of microbubbles. This waiting time is adequate for the formation of ligandcounter ligand bonds on the endothelium and for the uptake of part of freely circulating microbubbles by the reticuloendothelial system. After this time acquiring images with high $\mathrm{Ml}$ will resume. High MI imaging is then used throughout the image acquisition. The first acquired frame reflects the signal from both attached and freely circulating microbubbles. Imaging will continue 
by acquiring several short pulse interval frames to destroy attached microbubbles. Afterwards, a few long pulse interval frames will also be recorded. These frames represent the signal from freely circulating microbubbles, which keep flowing into the region of interest. Subtraction of the signal intensity from the averaged post-destruction frames from the initial pre-destruction frame will reflect the signal from retained microbubbles in the region of interest. This strategy is also illustrated on figure 13.

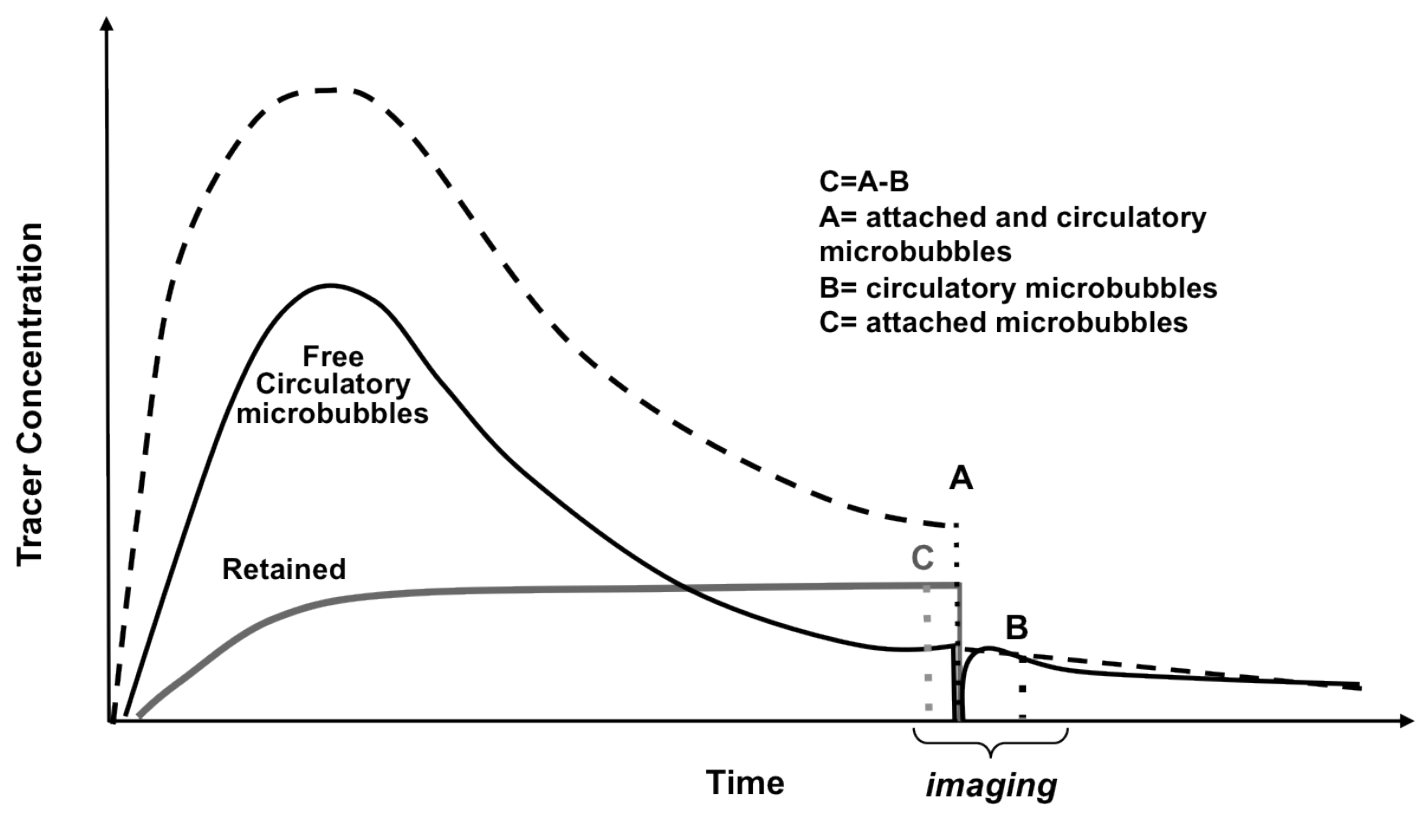

Figure 13) schematic depiction of the targeted contrast enhanced ultrasound imaging strategy. To obtain the signal from retained microbubbles $(C)$ in the region of interest the signal from post- destruction freely circulatory microbbubles (B) will be subtracted from the pre- destruction signal from both attached and freely circulatory micobubbles (A), A and $C$ are measured on ultrasound image. 


\subsection{Advantages and disadvantages of ultrasound based- imaging}

As mentioned before, molecular imaging has been developed for almost all types of imaging modalities available. The application of each imaging modality for a specific disease is influenced by different factors. Determinant factors could be sought in the type of detector and used contrast agent. In contrast enhanced ultrasound molecular imaging, microbubbles do not leave the vascular compartment thus limiting the potential targets into the vasculature. Additionally microbubble targeting and successful attachment follows the same rules as those for cell attachment. Therefore their attachment only happens at certain expression threshold of targeted molecules on the endothelial surface. However ultrasound based molecular imaging has some major advantages. First, in ultrasound molecular imaging there is a good balance between spatial resolution and the sensitivity of the technique in detecting the contrast agent. Second, the designed imaging protocols are relatively short and they can be completed in a period of 10 to 20 minutes. These are major characteristics, which programs implemented to screen large population would probably take advantage of. In addition Ultrasound molecular imaging could be of use in the fast detection of diseases such as coronary artery diseases, where time plays a critical role.

\subsection{Atherosclerosis}

Atherosclerosis is the main cause of coronary artery disease, cerebrovascular accidents and peripheral vascular disease, and consequently it represents the most common cause of morbidity and mortality globally. Atherosclerosis involves medium and large size arteries and is characterized by patchy subintimal infiltrates of the arterial wall. Fatty streaks are the earliest visible lesions of atherosclerosis and consist of lipid-laden foam cells accumulating in the intimal layer of arteries. Over time these lesions will evolve to fibrotic 
plaques encroaching into the arterial lumen. Plaques may become unstable resulting in plaque rupture and thrombotic occlusion of an artery. The extent of inflammation, oxidized lipid content, protease activation, plaque neovascularization and thrombus formation determine atherosclerotic plaque stability (42).

Inflammation plays a crucial role in the pathophysiology of atherosclerosis both in the initiation and progression of the disease. Biologically active species such as oxidized Low Density Lipoprotein (LDL), reactive oxygen species, and advanced glycation end products initiate the atherosclerotic process by stimulating vascular endothelial cells to produce and express inflammatory molecules. Inflammatory molecules such as Intercellular Adhesion Molecule (ICAM), Vascular Cell Adhesion Molecule (VCAM-1) and $P$-selectin facilitate the recruitment of inflammatory cells including monocytes and $T$ cells into the vessel walls by mediating interactions between leukocytes and the endothelium (43-45). Inflammatory cells promote smooth muscle cell migration and further recruitment of leukocytes by producing reactive oxygen species, vasoactive peptides, prothrombotic compounds, cytokines and proangiogenic growth factors (43). Therefore VCAM-1, ICAM-1 and/ or P-selctin could be used as potential markers for early detection of atherosclerosis.

\subsection{CEU imaging in atherosclerosis}

Ultrasound detection and enhancement of pathologic components of atherosclerosis could be achieved by targeting contrast agents to endothelial molecular markers of atherosclerotic lesions. Early in vitro studies demonstrated successful attachment of targeted microbubbles to ICAM-1 expressed on activated endothelial cells indicating the potential application of diagnostic ultrasound in characterizing cell phenotype (46).

First in vivo studies to assess the feasibility of ultrasound molecular imaging in detecting atherosclerosis encompassed application of intravascular ultrasound (IVUS) and intra-arterial injection of echogenic immunoliposomes. 
To create various stages of atheroma, yucatan miniswine carotid and femoral endothelial layers were injured and animals were kept on high-cholesterol diet. It was illustrated that there is a selective enhancement of molecular components of atheroma and injured endothelium by targeting liposomes to VCAM-1, ICAM-1, TF and fibrin. This allowed to better characterize the type and extent of atherosclerotic lesion progression (21). However, the invasive nature of IVUS imaging and required high doses of intra-arterial injected liposomes to produce signal above the background signal are a drawback in clinical translation of this method.

In another study to evaluate vascular inflammatory processes in atherosclerosis, non-invasive ultrasound molecular imaging using microbubbles was applied in a mouse model of atherosclerosis deficient for Apo-E lipoprotein. Given the fact that endothelial expression of VCAM-1 is up-regulated throughout the pathogenesis of atherosclerosis, microbubbles were targeted to VCAM-1. Contrast Enhanced Ultrasound imaging of the aortic arch demonstrated signal enhancement for microbubbles targeted to VCAM-1 but not for control microbubbles in atherosclerotic animals with advanced lesions. In contrast, no signal enhancement for microbubbles targeted to VCAM-1 was seen in control wild type animals that do not develop atherosclerosis. Therefore, CEU molecular imaging of VCAM-1 could noninvasively assess the degree of the development of atherosclerosis (19).

Likewise, CEU molecular imaging in atherosclerotic mice with genetic deletion for LDL receptor and apolipoprotein(Apo) mRNA editing protein Apobec-1 (LDLR ${ }^{-/} /$Apobec- ${ }^{-1-}$ ) could detect lesion-prone vascular phenotype before the appearance of advanced atheromas. The study showed an increased signal for VCAM-1 and P-selectin targeted microbubbles in ultrasound imaging at a timepoint when histologic studies demonstrated only mild regional vascular wall thickening consistent with very early stages of atherosclerosis. Accordingly the signal intensities at different age points were comparable with plaque development on histology (47).

CEU Molecular imaging has also been used to detect high-risk atherosclerotic phenotype by detecting activated von Willebrand Factor (VWF) on the vascular endothelium. Vascular collagen exposure to the circulation 
due to endothelial injury can cause conformational activation and adherence of VWF to endothelial cells. Interaction of VWF and activated platelets via glycoprotein-lba (GP- Iba) can initiate events that lead to thrombotic complications of atherosclerosis. Thus targeting of microbubbles to activated VWF by surface conjugation of GP- Iba has been used to trace thromboticprone atheromas in $\mathrm{LDLR}^{-/} /$Apobec- $1^{-1-}$ mice. On CEU, signal from VWF targeted microbubbles in the aortic arch of the atherosclerotic mice was significantly higher compared to the signal from wild type mice. En face microscopy of the aortic arch of the atherosclerotic mice also demonstrated co-localization of regions with adhered platelets and vWF microbubbles(42).

The aforementioned studies displayed the feasibility of CEU in non-invasively assessing atherosclerotic disease severity by targeting contrast agents to endothelial molecular components of atherosclerosis. As an extension of the spectrum of the use of molecular imaging in atherosclerosis, assessment of the response to drug treatment is of interest. 


\section{Aims of the PhD project}

The overall aim of this PhD project was to (1) investigate the influence of microbubble shell characteristics on targeting efficiency, and (2) to establish, whether ultrasound molecular imaging can be used to assess anti-inflammatory treatment effects of drugs in relevant murine models of atherosclerosis.

\subsection{Specific Aim 1}

To investigate the influence of the length of functionalized polyethylene glycol spacer arms incorporated in microbubble shell on improving the targeting efficiency of microbubbles

Polyethylene glycol tethers bearing a biotin molecule at their tip are used for attaching ligands for molecular imaging to the microbubble surface using a biotin-streptavidin-biotin link. The length of the polyethylene glycol tethers determines how far the ligands project away from the microbubble surface. Whether the length of polyethylene glycol tethers for ligand attachment influences attachment efficiency to molecular targets has never been examined. In addition, this effect may differ between molecular targets, as for example P-Selectin is a relatively long molecule that projects far away from the endothelial cell surface above the glycocalix, whereas ICAM-2 is a shorter molecule. Therefore in this research project we aimed to optimize the length of the polyethylene glycol tethers of the microbubble shell for targeted microbubble attachment to endothelial cells.

\subsection{Specific Aim 2}

To assess the feasibility of noninvasive ultrasound molecular imaging in detecting anti-inflammatory effect of statins in early murine atherosclerosis

Atherosclerosis is a chronic inflammatory disorder. Therefore therapies aimed at interrupting inflammatory signalling mechanisms or the inciting deposition of oxidized LDL in the vessel wall are being used therapeutically or being 
investigated (48). HMG-CoA reductase inhibitors (statins) have been shown to reduce VCAM-1 expression in experimental atherosclerosis $(49,50)$ independent of the effect on cholesterol, and have been studied extensively for primary and secondary prevention of cardiovascular events in clinical trials $(51,52)$. A method to noninvasively assess the reduction of VCAM-1 and other markers of inflammation in response to HMG-CoA might potentially be of use in assessing the adequacy of HMG-CoA dosing regimens. More importantly, non-invasive molecular imaging of the expression of VCAM-1 may be useful for selecting patients for emerging anti-inflammatory therapies and assessing the response to these agents. Therefore our aim was to assess the feasibility of ultrasound molecular imaging in detecting a reduction in endothelial expression of VCAM-1 in response to HMG-CoA.

\subsection{Specific Aim 3}

To investigate the short-term effects of treatment with apocynin on endothelial inflammation and thrombogenicity in a murine model of early atherosclerosis- application of ultrasound molecular imaging

Reactive Oxygen Species (ROS) have been attributed a key role in the inflammatory processes that lead to atherosclerosis. ROS products may also influence vascular thrombogenicity. Hence, there is an interest in antioxidant drugs as a treatment option. Apocynin is a small molecule that has been shown to inhibit endothelin-1-mediated VCAM-1 expression in the carotid arteries of hypertensive rats (53). Thus, our aim was to assess whether noninvasive ultrasound molecular imaging could detect the therapeutic effects of apocynin on vascular inflammatory activity and thrombogenicity. It is important to realize that a non-invasive method to assess vascular inflammation in vivo would be important in preclinical research for rapid, high throughput non-invasive assessment of drug effects. 


\section{Methods and material}

3.1 Factors affecting the endothelial retention of targeted microbbles: influence of mirobubble shell design and cell surface projection of the endothelial target molecule.

Elham Khanicheh MD, Martina Mitterhuber MSc, Katharina Kinslechner MSc, Lifen Xu PhD, Jonathan R. Lindner MD, Beat A. Kaufmann MD.

Journal of American Society of Echocardiography. 2012 Apr; 25(4):460-6 


\title{
Factors Affecting the Endothelial Retention of Targeted Microbubbles: Influence of Microbubble Shell Design and Cell Surface Projection of the Endothelial Target Molecule
}

Elham Khanicheh, MD, Martina Mitterhuber, MS, Katharina Kinslechner, BSc, Lifen Xu, PhD, Jonathan R. Lindner, MD, and Beat A. Kaufmann, MD, Basel, Switzerland; Portland, Oregon

\begin{abstract}
Background: In biologic systems, the arrest of circulating cells is mediated by adhesion molecules projecting their active binding domain above the cell surface to enhance bond formation and tether strength. Similarly, molecular spacers are used for ligands on particle-based molecular imaging agents. The aim of this study was to evaluate the influence of tether length for targeting ligands on ultrasound molecular imaging agents.

Methods: Microbubbles bearing biotin at the end of variable-length polyethylene glycol spacer arms $\left(\mathrm{MB}_{2000}\right.$ and $\mathrm{MB}_{3400}$ ) were prepared. To assess in vivo attachment efficiency to endothelial counterligands that vary in their distance from the endothelial cell surface, contrast-enhanced ultrasound (CEU) molecular imaging of tumor necrosis factor- $\alpha$-induced P-selectin (long distance) or intercellular adhesion molecule-2 (short distance) was performed with each agent in murine hind limbs. To assess the influence of the glycocalyx on microbubble attachment, CEU molecular imaging of intercellular adhesion molecule-2 was performed after degradation of the glycocalyx.
\end{abstract}

Results: CEU molecular imaging targeted to P-selectin showed signal enhancement above control agent for $\mathrm{MB}_{2000}$ and $\mathrm{MB}_{3400}$, the degree of which was significantly higher for $\mathrm{MB}_{3400}$ compared with $\mathrm{MB}_{2000}$. CEU molecular imaging targeted to intercellular adhesion molecule-2 showed low overall signal for all agents and signal enhancement above control for $\mathrm{MB}_{3400}$ only. Glycocalyx degradation increased signal for $\mathrm{MB}_{3400}$ and $\mathrm{MB}_{2000}$.

Conclusions: Microbubble targeting to endothelial ligands is influenced by (1) the tether length of the ligand, (2) the degree to which the endothelial target is projected from the cell surface, and (3) the status of the glycocalyx. These considerations are important for designing targeted imaging probes and understanding potential obstacles to molecular imaging. (J Am Soc Echocardiogr 2012;25:460-6.)

Keywords: Molecular imaging, Microbubbles, Contrast ultrasound, Microbubble design

Contrast-enhanced ultrasound (CEU) molecular imaging has been developed in recent years for the noninvasive assessment of pathophysiologic changes that are present on a molecular level in

From the Department of Biomedicine (E.K., M.M., K.K., L.X., B.A.K.) and Cardiology (B.A.K.), University Hospital Basel, Basel, Switzerland; and the Cardiovascular Division, Oregon Health \& Science University, Portland, Oregon (J.R.L.).

This study was supported by a SCORE grant (SNF 32323B_123819/1) from the Swiss National Science Foundation (Bern, Switzerland) to Dr. Kaufmann. Dr. Lindner is supported by grants R01-HL-078610, R01-DK-063508, and RC1-HL-100659 from the National Institutes of Health (Bethesda, Maryland). Dr. Khanicheh is supported by an MD-PhD startup grant from University Hospital Basel.

Reprint requests: Beat A. Kaufmann, MD, University Hospital Basel, Cardiology, Petersgraben 4, 4031 Basel, Switzerland (E-mail: kaufmannb@uhbs.ch). $0894-7317 / \$ 36.00$

Copyright 2012 by the American Society of Echocardiography.

doi:10.1016/j.echo.2011.12.016

460 atherosclerosis, myocardial ischemia, angiogenesis, thrombus formation, and heart transplant rejection. ${ }^{1-4} \mathrm{CEU}$ molecular imaging relies on the selective retention of targeted microbubbles within diseased tissue. ${ }^{5}$ Microbubble contrast agents are gas-filled, lipid-shelled or albumin-shelled structures that range from 1 to $4 \mu \mathrm{m}$ in diameter and are confined to the vascular compartment, thus restricting the potential targets to disease markers present on the endothelial cell surface. Targeting of lipid shelled microbubbles is achieved by conjugation of antibodies or other specific ligands to the microbubble shell surface via functionalized lipids. Targeted retention of microbubbles in a vessel under any given shear condition depends on a number of factors, including (1) ligand density on the microbubble surface, ${ }^{6}$ (2) ligand-specific bond kinetics, and (3) site density of the molecular target. ${ }^{7}$ Similar to the case with leukocyte and platelet adhesion molecules that have their binding domains projected away from the cell surface to enhance bond formation, ${ }^{8}$ the active ligand on targeted microbubbles is often placed at the end of polyethylene glycol (PEG) multimers that project the ligand away from the shell surface and beyond any PEG brush that has been added to the shell surface to stabilize microbubbles. 


\begin{tabular}{|l|}
\hline \multicolumn{1}{|c|}{ Abbreviations } \\
\hline CEU = Contrast-enhanced \\
ultrasound \\
FITC = Fluorescein \\
isothiocyanate \\
ICAM = Intercellular adhesion \\
molecule \\
PEG = Polyethylene glycol \\
TNF = Tumor necrosis factor \\
\hline
\end{tabular}

The aim of this study was to assess whether the length of the functionalized PEG molecules used to conjugate antibodies to the microbubble shell surface influences targeting efficiency. The importance of this issue is underscored by the desire to target not only long adhesion molecules that project their binding moiety far away from the endothelial cell surface and beyond the glycocalyx but also smaller molecules that reside closer to the endothelial cell surface. We therefore compared the targeting efficiency of microbubbles with differentlength functionalized PEG molecules to adhesion molecules with a long-distance (P-selectin) and short-distance (intercellular adhesion molecule [ICAM]-2) projection from the endothelial surface, with and without modification of the glycocalyx.

\section{METHODS}

\section{Microbubble Preparation}

Lipid-shelled decafluorobutane microbubbles were prepared by sonication of a gas-saturated aqueous suspension of distearoylphosphatidylcholine $(2 \mathrm{mg} / \mathrm{mL})$ and polyoxyethylene- 40 -stearate $(1 \mathrm{mg} / \mathrm{mL})$. For ligand conjugation, functionalized PEG-lipid conjugates containing biotin at the PEG N-terminus were added to the aqueous suspension at concentrations corresponding to equal molar ratios of 50:10:1 (distearoylphosphatidylcholine/polyoxyethylene-40-stearate/functionalized PEG). The PEG length was varied by using 1,2-distearoyl-sn-glycerophosphoethanolamine-N-[biotinyl(PEG)-2000] (Avanti Polar Lipids, Alabaster, AL), 1,2-distearoyl-sn-glycero-phosphoethanolamine-N[biotinyl(PEG)-3400] (Creative PEG Works, Winston-Salem, NC), or 1,2-distearoyl-sn-glycero-phosphoethanolamine-N-[biotinyl(PEG)10000] (Nanocs Inc., Boston, MA) Thus, microbubbles with

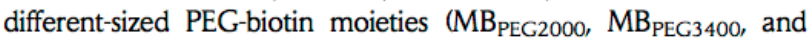
$M_{\text {PEG } 10000}$ ) were prepared with PEG multimers of 45,80 , and 225 , respectively.

Monoclonal antibodies were conjugated to the microbubble surface as previously described using a streptavidin-biotin link. ${ }^{9}$ Rat antimouse monoclonal immunoglobulin G1 against either P-selectin (Rb40.34) or ICAM-2 (mLC2/4) were conjugated to the microbubble surface to produce P-selectin-targeted (MB PEG2000-PSel, MB $_{\text {PEC3400-PSel, and MB }}$ PEG 10000-PSel $)$ or ICAM-2-targeted (MB PEG 2000-ICAM, MB $_{\text {PEG3400-ICAM, and MB }}$ PEG10000-ICAM) microbubbles, each with different-length PEG-biotin moieties. Isotype control antibodies (R3-34; BD Bioscience, San Jose, CA) were conjugated to the surfaces of microbubbles with randomly selected PEG spacer-length moieties to produce control microbubbles $\left(\mathrm{MB}_{\mathrm{Ctr}}\right)$. Microbubble concentration, size, and surface area were measured by electrozone sensing (Multisizer III; Beckman Coulter, Brea, CA).

\section{Biotin and Antibody Density on the Microbubble Surface}

To quantify the number of PEG spacer moieties and antibodies on the microbubble surface by quantitative fluorescence, aliquots of $1 \times 10^{8}$ for $\mathrm{MB}_{\mathrm{PEC} 2000}, \mathrm{MB}_{\mathrm{PEG} 3400}$, and $\mathrm{MB}_{\mathrm{PEG} 10000}$ were washed and incubated with $30 \mu \mathrm{g}$ fluorescein isothiocyanate (FITC)-labeled streptavidin (Leinco Technologies, St. Louis, MO). Excess FITC-streptavidin was removed with three cycles of flotation centrifugation.
Microbubble concentration and mean surface area were measured, and the microbubbles were destroyed by prolonged application of pressure at $100 \mathrm{~mm} \mathrm{Hg}$. The concentration of fluorescently labeled streptavidin was measured using a Gemini XPS fluorescence microplate reader (Molecular Devices, Sunnyvale, CA) and compared with a concentration-intensity reference standard to derive the density per surface area. For determination of the number of antibodies bound per square micrometer of microbubble surface, a known concentration of biotinylated immunoglobulin $G$ was labeled with FITC. Labeled antibodies were conjugated to the different microbubble preparations, and quantified as described for FITC-streptavidin. All experiments were performed in triplicate.

\section{Animal Preparation}

All experiments were performed in accordance with Swiss federal legislation and were approved by the local animal care and use committee. Male wild-type C57BL/6 mice aged 8 to 10 weeks were anaesthetized with $1.5 \%$ inhaled isoflurane. Body temperature was maintained at $37^{\circ} \mathrm{C}$ with a heating pad. A jugular vein was cannulated for administration of microbubbles.

\section{CEU Imaging}

Ultrasound imaging (Sequoia Acuson C512; Siemens Medical Systems USA Inc., Mountain View, CA) was performed with a high-frequency linear-array probe (15L8) held in place by a railed gantry system. The adductor muscle of the mouse hind limb was imaged in short axis. Anatomic images of the hind limb were acquired with fundamental imaging at $14 \mathrm{MHz}$. CEU was performed with power modulation and pulse inversion (Contrast Pulse Sequence) imaging at a centerline frequency of $7 \mathrm{MHz}$. The gain settings were adjusted just below visible tissue speckle and held constant.

In vivo circulation half-life of microbubble preparations with different length PEG spacer moieties were compared using low-mechanical index ultrasound imaging. Nontargeted $\mathrm{MB}_{\mathrm{PEC} 2000}, \mathrm{MB}_{\mathrm{PEC} 3400}$, and $\mathrm{MB}_{\mathrm{PEG} 10000}\left(1 \times 10^{7}\right.$ each $)$ were injected intravenously as a bolus in random order. Thirty seconds after the bolus injection, single image frames of the proximal hind limb were obtained at a mechanical index of 0.2 at 15 -sec intervals for $10 \mathrm{~min}$. It has previously been shown that these imaging parameters do not result in microbubble destruction when imaging the mouse hind limb. ${ }^{10}$ On digitized image sequences, acoustic intensity from a region of interest over the hind limb adductor muscle was derived over time after removal of logarithmic signal compression.

For in vivo assessment of attachment efficiency of microbubbles targeted to P-selectin with different-length PEG spacer moieties, a mouse model of murine hind limb inflammation was used $(n=$ 16). CEU molecular imaging of the hind limb was performed 45 min after intramuscular injection of tumor necrosis factor (TNF)$\alpha$ (250 ng). MB PEG2000-PSel, MB $B_{\text {PEG 3400-PSel, }}$ MB PEG 10000-PSel, and $\mathrm{MB}_{\mathrm{Ctr}}\left(1 \times 10^{7}\right.$ each) were injected intravenously as a bolus in random order. Ultrasound imaging was paused for $8 \mathrm{~min}$ after each injection. Imaging was then resumed at a mechanical index of 0.87 . The first acquired image frame was used to derive the total amount of microbubbles present in tissue. The microbubbles in the ultrasound beam were then destroyed with several $(>10)$ image frames. Several image frames at a long pulsing interval ( $10 \mathrm{sec}$ ) acquired after microbubble destruction were then acquired to measure signal attributable to freely circulating microbubbles. Data were log-linear converted, and frames representing freely circulating microbubbles were digitally subtracted from the first image frame to derive signal from attached microbubbles alone. ${ }^{9}$ 

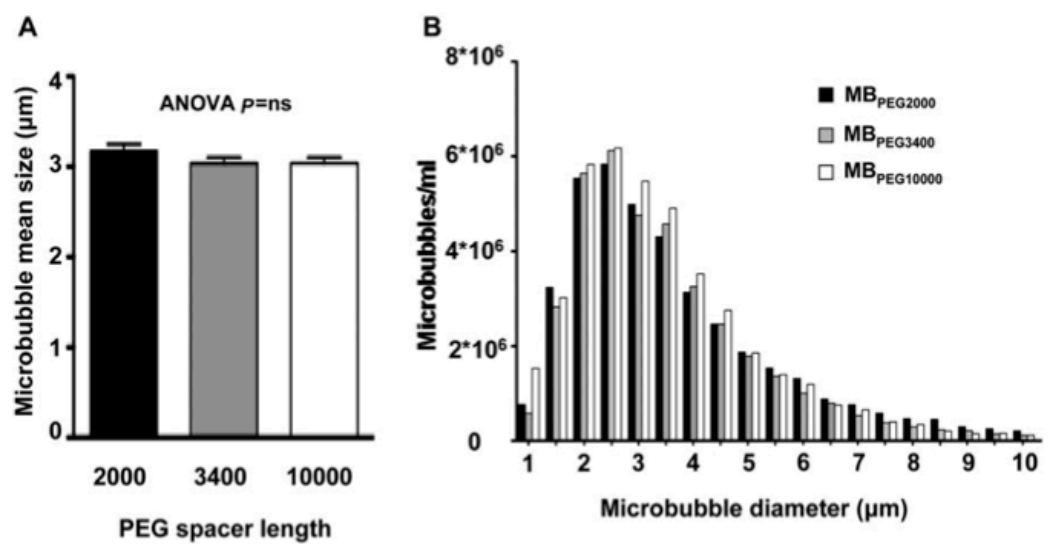

Figure 1 (A) Mean \pm SD diameter for microbubble preparations containing PEG spacer molecules with a PEG 2000, PEG 3400, or PEG 10000 spacer length. Data are from $\geq 10$ microbubble preparations for each PEG spacer length. (B) Histograms illustrating the size distribution of the three microbubble preparations. ANOVA, Analysis of variance.

For in vivo assessment of the attachment efficiency of microbubbles targeted to ICAM-2, imaging was performed as described above in murine hind limbs $(n=16)$, without induction of inflammation, because ICAM-2 has been shown to be constitutively expressed on endothelial cells, with the level of expression not influenced by cytokines. ${ }^{11,12}$ In an additional 16 mice, molecular imaging was performed $60 \mathrm{~min}$ after intravenous injection of $32 \mathrm{IU}$ of Streptomyces hyaluronidase (Sigma-Aldrich, St. Louis, MO), which was performed to reduce microvascular endothelial glycocalyx thickness. ${ }^{13}$

\section{Statistical Analysis}

Data were analyzed using GraphPad Prism version 5.0d (GraphPad Software, Inc., La Jolla, CA). Data are expressed as mean \pm SEM unless stated otherwise. One-way analysis of variance with Tukey's post hoc test was used for multiple comparisons of normally distributed variables. Friedman's repeated-measures analysis of variance with Dunn's post hoc test was used to compare variables not normally distributed. Two-sided $P$ values $<.05$ were considered statistically significant.

\section{RESULTS}

\section{Microbubble Shell Characteristics}

Because microbubble acoustic signal is influenced by size, ${ }^{14}$ microbubble diameter was assessed for the different agents according to PEG spacer length. The microbubble agents did not differ in terms of their mean diameters or their size distributions (Figure 1), with $10 \pm 6 \%, 8 \pm 4 \%$, and $8 \pm 5 \%$ of $\mathrm{MB}_{\mathrm{PEC} 2000}, \mathrm{MB}_{\mathrm{PEC} 3400}$, and $\mathrm{MB}_{\mathrm{PEG} 10000}$, respectively, having diameters $>5 \mu \mathrm{m}(P=\mathrm{NS}$ for multiple comparison).

Fluorescent labeling with streptavidin showed similar numbers of biotin molecules on the microbubble surface for $\mathrm{MB}_{\mathrm{PEC} 2000}$ and $\mathrm{MB}_{\mathrm{PEC} 3400}$ but a significantly lower number of biotin molecules for $M B_{P E G 10000}$ (Table 1). As a result, surface density for antibody that was coupled via biotin was similar for $\mathrm{MB}_{\mathrm{PEC} 2000}$ and $\mathrm{MB}_{\mathrm{PEC} 3400}$ but was more than an order of magnitude lower for $\mathrm{MB}_{\mathrm{PEG} 10000}$.

\section{In Vivo Circulation Time}

Because the duration of recirculation could potentially affect the extent of agent attachment, microbubble circulation life span was assessed in vivo using nondestructive low-mechanical index ultra-
Table 1 PEG-biotin and antibody surface density

\begin{tabular}{lcc}
\hline PEG spacer length & $\begin{array}{c}\text { PEG-biotin surface } \\
\text { density }\left(\mu \mathrm{m}^{-2}\right)\end{array}$ & $\begin{array}{c}\text { Antibody surface } \\
\text { density }\left(\mu \mathrm{m}^{-2}\right)\end{array}$ \\
\hline PEG 2000 & $12,463 \pm 1,834$ & $4,052 \pm 1,480$ \\
PEG 3400 & $14,799 \pm 5,567$ & $3,703 \pm 1,762$ \\
PEG 10000 & $2,173 \pm 2,349$ & $176 \pm 120$ \\
\hline
\end{tabular}

sound imaging. The time intensity profile and circulation half-life were similar for $\mathrm{MB}_{\mathrm{PEG} 2000}, \mathrm{MB}_{\mathrm{PEC} 3400}$, and $\mathrm{MB}_{\mathrm{PEG} 10000}$ (Figure 2).

\section{Influence of PEG-Spacer Length on Attachment}

CEU molecular imaging for P-selectin in TNF- $\alpha$-induced hind limb inflammation showed selective signal enhancement for $\mathrm{MB}_{\mathrm{PEC} 2000-\mathrm{PSel}}$ and $\mathrm{MB}_{\mathrm{PEC} 3400-\mathrm{PSel}}$ compared with control microbubbles (Figure 3). Signal enhancement for $\mathrm{MB}_{\mathrm{PEC} 3400-\mathrm{PSel}}$ was approximately $30 \%$ higher than for $\mathrm{MB}_{\mathrm{PEC} 2000-\mathrm{PSel}}$. Signal for $\mathrm{MB}_{\mathrm{PEG} 10000 \text {-PSel }}$ was not significantly different from control microbubbles, although there was a trend toward selective signal enhancement. The signal from control microbubbles was not dependent on the PEG spacer length that was used (signal for $\mathrm{MB}_{\text {PEC2000-Ctr }} 0.50 \pm 0.14$, for $\mathrm{MB}_{\mathrm{PEC} 3400 \text {-Ctr }} 0.53 \pm$ 0.17 , and for $\mathrm{MB}_{\mathrm{PEG} 10000-\mathrm{Ctr}_{\mathrm{T}}} 0.35 \pm 0.13 ; P=\mathrm{NS}$ ).

CEU molecular imaging for ICAM-2, which has a shorter projection distance for the active binding site from the endothelial cell surface, showed very low signals overall for all agents. However, there was selective signal enhancement for MB $\mathrm{BEG}_{\mathrm{P}} 400-\mathrm{ICAM}$ but not for

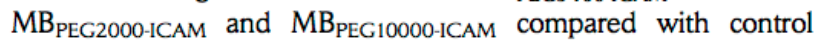
microbubbles (Figure 4). Signal intensity for $M_{\text {PEG 3400-ICAM was }}$ twofold higher than for MBPEC2000-ICAM.

After glycocalyx degradation, the signal increased for both

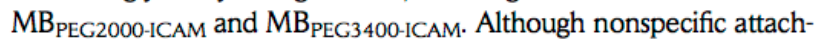
ment of $\mathrm{MB}_{\mathrm{Ctr}}$ increased as well, this signal was significantly lower than that for $\mathrm{MB}_{\mathrm{PEC} 2000-\mathrm{ICAM}}$ and $\mathrm{MB}_{\mathrm{PEG} 3400-\mathrm{ICAM} \text {. }}$

\section{DISCUSSION}

The aim of this study was to assess the influence of different-length functionalized PEG molecules for targeting ligand attachment on in vivo contrast-enhanced molecular imaging signals. We show that 


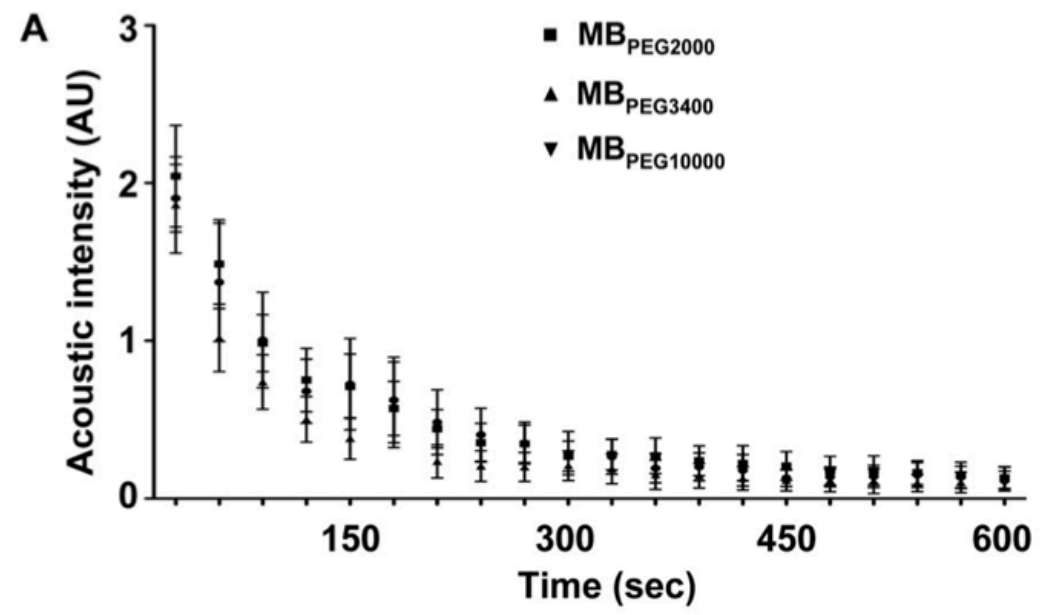

B

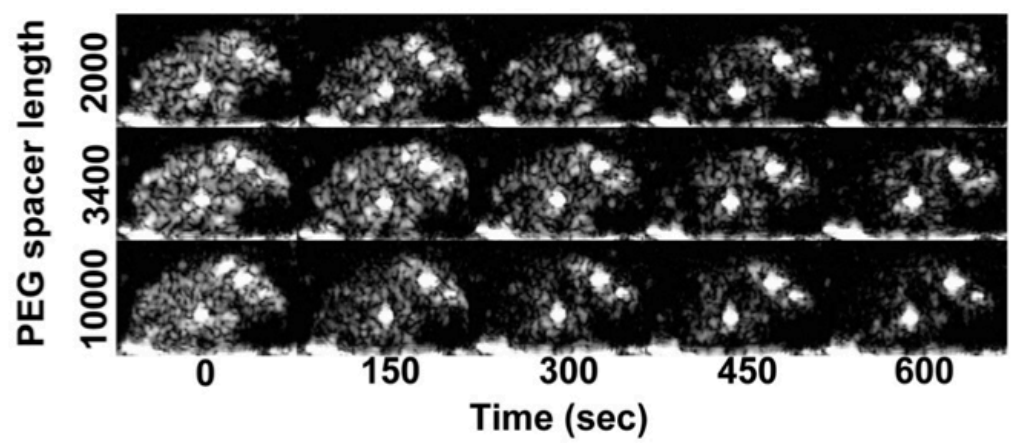

C

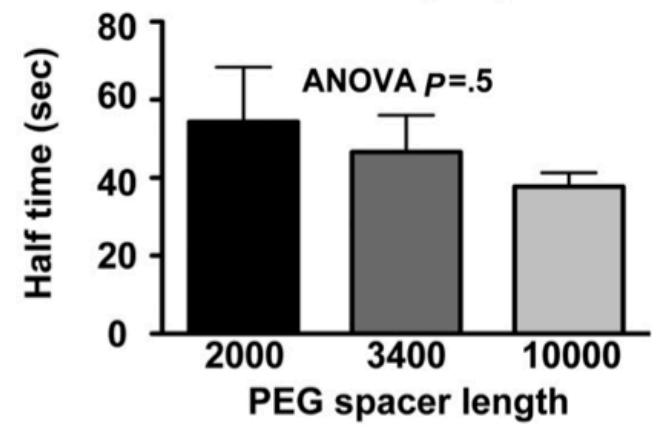

Figure 2 CEU data used to evaluated recirculation time for the microbubble agents that varied according to PEG length. (A) Time versus mean \pm SEM video intensity plots on CEU used to assess the duration of recirculation for the three microbubble preparations ( $n=5$ mice with injection of the three microbubble preparations in random order). (B) Examples of CEU images at incremental intervals after bolus injection of the three microbubble preparations. Time intervals after bolus injection are provided at the bottom of each image. (C) Mean \pm SEM circulation half-life for each agent. $P=$ NS between the three agents. ANOVA, Analysis of variance.

the use of longer $\left(\mathrm{PEG}_{3400}\right)$ functionalized PEG molecules to target adhesion molecules on the endothelial surface results in better attachment efficiency and thus better signal enhancement than the use of shorter $\left(\mathrm{PEG}_{2000}\right.$ ) functionalized PEG molecules. Microbubbles with a $P E G_{10000}$ chain, which was not incorporated well into the shell surface, yielded signals that were consistently lower than for $\mathrm{MB}_{\mathrm{PEC} 3400}$ and $\mathrm{MB}_{\mathrm{PEC} 2000}$, confirming earlier in vitro results that found a significant impact of microbubble surface antibody density on targeted adhesion to endothelial cells. ${ }^{6}$

For ultrasound molecular imaging, targeted microbubble attachment occurs under flow conditions, with only milliseconds available for bond formation between the receptor and the targeting ligand.
Once formed, the bonds have to withstand shear forces acting on the microbubble at the bloodstream-endothelial interface. In addition, the glycocalyx constitutes a physical barrier to the interaction of particle-based ligands with endothelial targets. Leukocytes accomplish attachment to endothelial cells in a remarkably efficient and specific manner. The initial tethering of leukocytes is conveyed by the interaction of selectins with cell-surface glycoconjugates. Both selectins and their glycoconjugate ligands are unusually long molecules, ${ }^{8}$ and the lengths of selectins and their ligands have an impact on leukocyte rolling. ${ }^{15,16}$ Thus, targeted microbubbles are designed with the ligands attached to the distal tips of functionalized PEG spacer arms with the goal of emulating leukocytes. Mimicry of such 

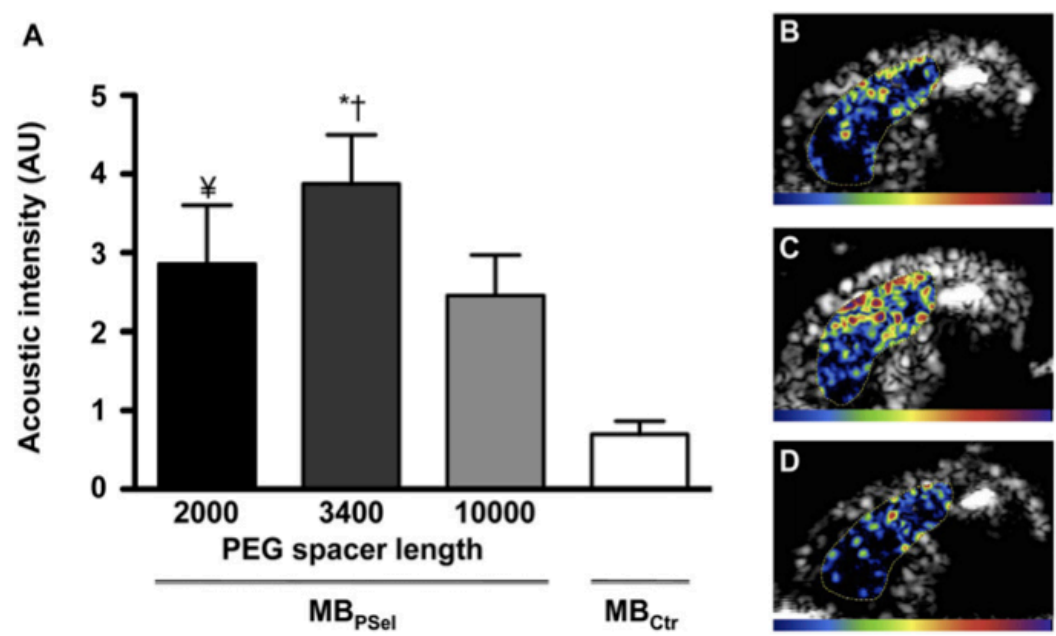

Figure 3 Molecular imaging data for the three microbubble preparations targeted to P-selectin $(n=16$ mice). (A) Mean \pm SEM of background-subtracted targeted signal intensity for the three microbubble preparations bearing a P-selectin antibody and microbubbles bearing a control antibody. Examples of background-subtracted color-coded images of targeted signal from one animal are

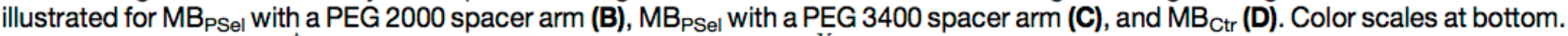
${ }^{\star} P<.001$ versus $\mathrm{MB}_{\mathrm{Ctr}},{ }^{\dagger} P<.01$ versus PEG 2000 and PEG 10000; ${ }^{¥} P<.01$ versus $\mathrm{MB}_{\mathrm{Ctr}}$.
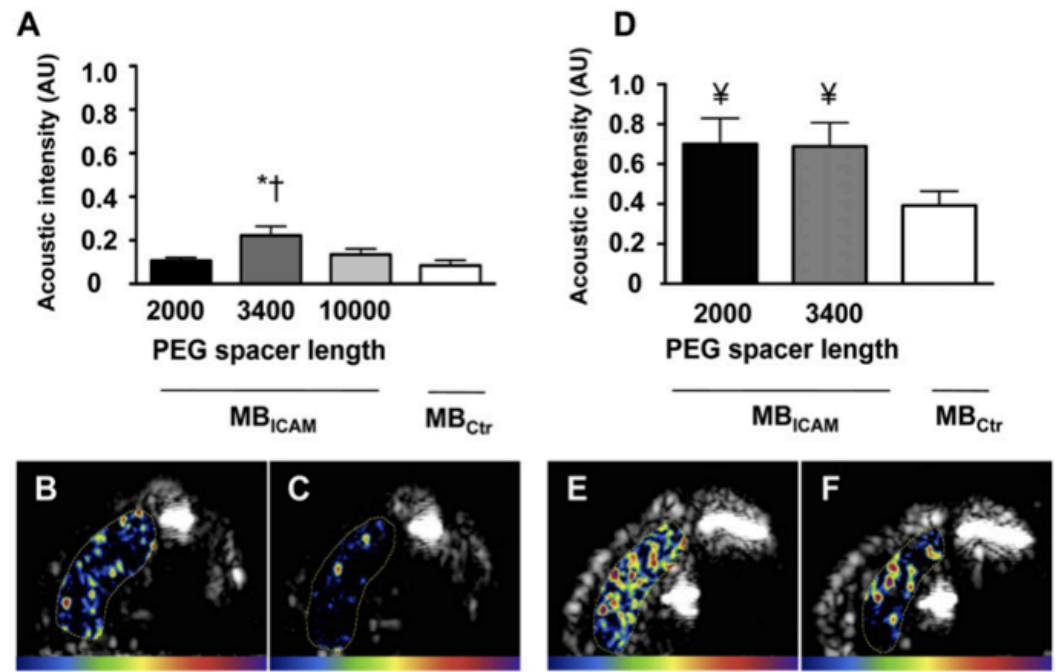

Figure 4 Molecular imaging data for microbubble preparations targeted to ICAM-2 in murine hind limb skeletal muscle. (A) Mean \pm SEM of background-subtracted targeted signal intensity for the three microbubble preparations bearing an ICAM-2 antibody and microbubbles bearing a control antibody ( $n=16$ mice). Examples of background-subtracted color-coded images of targeted signal from one animal are illustrated for $\mathrm{MB}_{\mathrm{ICAM}}$ with a PEG 3400 spacer arm (B) and $\mathrm{MB}_{\mathrm{Ctr}}$ (C). (D) Mean $\pm \mathrm{SEM}$ of background-subtracted signal intensity for $\mathrm{MB}_{\mathrm{PEG} 3400-\mathrm{ICAM}}, \mathrm{MB}_{\mathrm{PEG} 2000-\mathrm{ICAM}}$, and $\mathrm{MB}_{\mathrm{Ctr}} 60 \mathrm{~min}$ after intravenous injection of hyaluronidase $(n=16)$. Examples of background-subtracted color-coded images of targeted signal from one animal are illustrated for MB $_{\text {ICAM }}$ with a PEG 2000 spacer $\operatorname{arm}(\mathbf{E})$ and $\mathrm{MB}_{\mathrm{Ctr}}$ (F). ${ }^{\star} P<.01$ versus $\mathrm{MB}_{\mathrm{Ctr}} ;{ }^{\dagger} P<.05$ versus $\mathrm{PEG} 2000 ;{ }^{¥} P<.05$ versus $\mathrm{MB}_{\mathrm{Ctr}}$.

targeting mechanisms on in vitro experiments with microparticles targeted to P-selectin has shown an increased microparticle binding frequency and increased adhesive lifetimes with ligands attached to longer PEG spacer molecules. ${ }^{17}$

In the present study, we incorporated different-length PEG spacers functionalized with biotin for ligand attachment into the lipid shell of decafluorobutane microbubbles. Equimolar dissolution of $\mathrm{PEG}_{2000}$ and $\mathrm{PEG}_{3400}$ spacers resulted in equivalent numbers of spacer arms available for streptavidin binding on the microbubble surface. In contrast, the number of $\mathrm{PEG}_{10000}$ spacer arms on the microbubble surface available for streptavidin binding was seven to eight times lower at equimolar concentrations of the $\mathrm{PEG}_{10000}$ spacer, and the relative reduction in the density of $\mathrm{mAb}$ conjugated to the surface was even lower. Incorporation of $\mathrm{PEG}_{10000}$ was not increased when using higher molecular ratios (data not shown). A larger extent of polymer chain collapse may have impeded integration of the longer and thus bulkier PEG ${ }_{10000}$ chains into the microbubble surface at sufficient densities because of spatial constraints and the increased repulsion between larger individual molecules, an effect also referred to as steric hindrance.

When polymers are grafted on a surface at low density, the individual polymer chains will curl up and form mushroom-like structures. As 
the grafting density is increased, the polymer chains start to repel each other and extend away from the grafting surface in a brush-like fashion. On the basis of the number of functionalized PEG spacer chains per square micrometer derived from fluorescence labeling experiments and the 10:1 molar ratio of PEG-stearate to functionalized PEG spacer, it can safely be assumed that a brush confirmation was obtained for $\mathrm{MB}_{\mathrm{PEC} 2000}$ and $\mathrm{MB}_{\mathrm{PEC} 3400 .}{ }^{18}$ In this configuration, it has been estimated that true lengths of $\mathrm{MB}_{\mathrm{PEC} 2000}$ and $\mathrm{MB}_{\mathrm{PEC} 3400}$ at the measured site densities would be about 10 and $18 \mathrm{~nm}$, respectively, corresponding to about $70 \%$ of the maximal length of the PEG chains. ${ }^{18,19}$

In vivo ultrasound molecular imaging showed selective signal enhancement for both $\mathrm{MB}_{\mathrm{PEC} 3400}$ and $\mathrm{MB}_{\mathrm{PEC} 2000}$ targeted to P-selectin but only for $\mathrm{MB}_{\mathrm{PEC} 3400}$ targeted to ICAM-2. Overall, signal enhancement was strong for both $\mathrm{MB}_{\mathrm{PEC} 3400}$ and $\mathrm{MB}_{\mathrm{PEC} 2000}$ targeted to P-selectin, which projects its binding domain about $40 \mathrm{~nm}$ away from the endothelial cell surface. ${ }^{20}$ In contrast, and despite a high level of constitutive expression of ICAM- 2 on the endothelium that has been reported to be even higher than TNF- $\alpha$-stimulated expression of P-selectin, ${ }^{12}$ signal enhancement was weak for $\mathrm{MB}_{\mathrm{PEC} 3400}$ and $\mathrm{MB}_{\mathrm{PEC} 2000}$ targeted to ICAM-2. We believe this was because the adhesion molecule extends $<10 \mathrm{~nm}$ from the endothelial cell surface. ${ }^{21,22}$ In both animal models, signal from MB PEC3400 was significantly higher than signal from $\mathrm{MB}_{\mathrm{PEC} 2000}$, indicating advantages of the longer PEG spacer arms for binding efficiency. ${ }^{6}$

The mechanism for increased binding efficiency when using a longer PEG spacer arm may result from better accessibility to the target. In addition, it has been shown that longer PEG spacer arms may also improve targeting efficiency by increasing the bond lifetime of microparticles under flow conditions by preferentially localizing the bond structure at the trailing end of the microparticle, thus reducing torque forces acting on the microparticle under flow conditions. ${ }^{17}$

Microbubble interaction with endothelial targets occurs on the endothelial cell surface covered by the glycocalyx. Recent data suggest that the glycocalyx measures up to $500 \mathrm{~nm}$ in normal microcirculatory venules. ${ }^{23}$ The low signal for both $M_{\text {PEG } 3400-I C A M}$ and

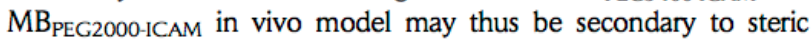
hindrance and/or charge repulsion caused by the glycocalyx, which lowered accessibility of the microbubbles to the target. Still, differences in PEG spacer length seemed to retain an influence on attachment efficiency, possibly by increasing the chance of a microbubble for deploying the targeting ligands close enough to the endothelial cell surface for bond formation. Degradation of the glycocalyx lead to increased retention of $M_{\text {PEG 3400-ICAM }}$ and MB $_{\text {PEG 2000-ICAM but }}$ also to increased nonspecific attachment of $\mathrm{MB}_{\mathrm{Ctr}}$ This may have been secondary to complement-mediated attachment to either endothelial cells or to adherent leukocytes as a result of hyaluronidase treatment. $^{24,25}$

Several limitations of this study should be mentioned. We prepared microbubbles with three different PEG spacers carrying a biotin moiety for ligand conjugation and noted a reduced incorporation of the PEG 10000 spacer molecule. However, whether incorporation of intermediate length PEG spacers (eg, PEG 5000) would be possible was not tested.

On in vivo experiments, comparisons of the targeting efficiency of different microbubble species (e.g., $\mathrm{MB}_{2000}$ vs $\mathrm{MB}_{3400}$ vs $\mathrm{MB}_{10000}$ ) were made within identical models and with identical targeting antibodies. Thus, targeted imaging signals can be compared safely among microbubble preparations with different-length PEG spacers. In contrast, comparison between the two in vivo models is not only influenced by targeting efficiency but could also be dependent on the properties of the two different antibodies used for targeting and differences in the site density of the target molecule. However, given the reported high level of constitutive expression of ICAM-2 on endothelial cells in different murine tissues ${ }^{12}$ and the large difference in targeted signals for molecular imaging of P-selectin versus ICAM-2, we still think that it is reasonable to conclude that the overall lower targeting efficiency for ICAM-2 is due to lower accessibility.

Also, in our study, we used monoclonal antibodies as ligands. Whether humanized antibodies or smaller peptide ligands will ultimately be used for translation into clinical trials of ultrasound molecular imaging is uncertain, and the specific properties of smaller peptide ligands conjugated to different length spacers would have to be tested.

Last, we did not measure directly the glycocalyx thickness in our two different mouse models. However, it has been well documented that TNF- $\alpha$-induced inflammation leads to an almost complete degradation of the glycocaly $\mathrm{x}^{23}$ and that systemic application of hyaluronidase reduces glycocalyx thickness. ${ }^{13}$

\section{CONCLUSIONS}

We have shown that the microbubble architecture, specifically the length of PEG spacer moieties used for conjugation of targeting ligands, has a significant impact on targeting efficiency. These results have an implication for the shell design of targeted microbubbles. In addition, reduced accessibility due to the thickness of the glycocalyx in the vessels of interest may have an important influence on microbubble attachment. This observation could have implications for the selection of potential molecular imaging targets.

\section{REFERENCES}

1. Kaufmann BA, Carr CL, Belcik JT, Xie A, Yue Q, Chadderdon S, et al. Molecular imaging of the initial inflammatory response in atherosclerosis: implications for early detection of disease. Arterioscler Thromb Vasc Biol 2010;30:54-9.

2. Kaufmann BA, Lindner JR. Molecular imaging with targeted contrast ultrasound. Curr Opin Biotechnol 2007;18:11-6.

3. Kaufmann BA, Lewis C, Xie A, Mirza-Mohd A, Lindner JR. Detection of recent myocardial ischaemia by molecular imaging of P-selectin with targeted contrast echocardiography. Eur Heart J 2007;28:2011-7.

4. Behm CZ, Kaufmann BA, Carr C, Lankford M, Sanders JM, Rose CE, et al. Molecular imaging of endothelial vascular cell adhesion molecule-1 expression and inflammatory cell recruitment during vasculogenesis and ischemia-mediated arteriogenesis. Circulation 2008;117:2902-11.

5. Kaufmann BA. Ultrasound molecular imaging of atherosclerosis. Cardiovasc Res 2009;83:617-25.

6. Weller GE, Villanueva FS, Klibanov AL, Wagner WR. Modulating targeted adhesion of an ultrasound contrast agent to dysfunctional endothelium. Ann Biomed Eng 2002;30:1012-9.

7. Takalkar AM, Klibanov AL, Rychak JJ, Lindner JR, Ley K. Binding and detachment dynamics of microbubbles targeted to P-selectin under controlled shear flow. J Control Release 2004;96:473-82.

8. Li F, Erickson HP, James JA, Moore KL, Cummings RD, McEver RP. Visualization of $\mathrm{P}$-selectin glycoprotein ligand-1 as a highly extended molecule and mapping of protein epitopes for monoclonal antibodies. J Biol Chem 1996;271:6342-8.

9. Lindner JR, Song J, Christiansen J, Klibanov AL, Xu F, Ley K. Ultrasound assessment of inflammation and renal tissue injury with microbubbles targeted to P-selectin. Circulation 2001;104:2107-12.

10. Kaufmann BA, Carr CL, Belcik T, Xie A, Kron B, Yue Q, et al. Effect of acoustic power on in vivo molecular imaging with targeted microbubbles: implications for low-mechanical index real-time imaging. J Am Soc Echocardiogr 2010;23:79-85. 
11. Nortamo P, Li R, Renkonen R, Timonen T, Prieto J, Patarroyo M, et al. The expression of human intercellular adhesion molecule- 2 is refractory to inflammatory cytokines. Eur J Immunol 1991;21:2629-32.

12. Langley RR, Russell J, Eppihimer MJ, Alexander SI, Gerritsen M Specian RD, et al. Quantification of murine endothelial cell adhesion molecules in solid tumors. Am J Physiol 1999;277:H1156-66.

13. Henry CB, Duling BR. Permeation of the luminal capillary glycocalyx is determined by hyaluronan. Am J Physiol 1999;277:H508-14.

14. de Jong N, Bouakaz A, Frinking P. Basic acoustic properties of microbub bles. Echocardiography 2002;19:229-40.

15. Yago T, Leppanen A, Qiu H, Marcus WD, Nollert MU, Zhu C, et al. Distinct molecular and cellular contributions to stabilizing selectinmediated rolling under flow. J Cell Biol 2002;158:787-99.

16. Patel KD, Nollert MU, McEver RP. P-selectin must extend a sufficient length from the plasma membrane to mediate rolling of neutrophils. J Cell Biol 1995;131:1893-902.

17. Ham AS, Klibanov AL, Lawrence MB. Action at a distance: lengthening adhesion bonds with poly(ethylene glycol) spacers enhances mechanically stressed affinity for improved vascular targeting of microparticles. Langmuir 2009;25:10038-44.
18. Naji A, Seidel C, Netz RR. Theoretical approaches to neutral and charged polymer brushes. Adv Polym Sci 2006;198:149-83.

19. Borden MA, Zhang H, Gillies RJ, Dayton PA, Ferrara KW. A stimulusresponsive contrast agent for ultrasound molecular imaging. Biomaterials 2008;29:597-606

20. Bevilacqua MP, Nelson RM. Selectins. J Clin Invest 1993;91:379-87.

21. Carlos TM, Harlan JM. Leukocyte-endothelial adhesion molecules. Blood 1994;84:2068-101.

22. Springer TA. Adhesion receptors of the immune system. Nature 1990; 346:425-34.

23. Potter DR, Jiang J, Damiano ER. The recovery time course of the endothelial cell glycocalyx in vivo and its implications in vitro. Circ Res 2009;104: 1318-25.

24. Khan AI, Kerfoot SM, Heit B, Liu L, Andonegui G, Ruffell B, et al. Role of cd44 and hyaluronan in neutrophil recruitment. J Immunol 2004;173: 7594-601.

25. Anderson DR, Tsutsui JM, Xie F, Radio SJ, Porter TR. The role of complement in the adherence of microbubbles to dysfunctional arterial endothelium and atherosclerotic plaque. Cardiovasc Res 2007;73: 597-606. 
3.2 Noninvasive ultrasound molecular imaging of the effects of Statins on endothelial inflammatory phenotype in early atherosclerosis.

Elham Khanicheh MD, Martina Mitterhuber MSc, Lifen Xu PhD, Stéphanie P. Haeuselmann MSc, Gabriela M. Kuster MD, Beat A. Kaufmann MD

PLoS ONE 8(3): e58761, 2013 


\title{
Noninvasive Ultrasound Molecular Imaging of the Effect of Statins on Endothelial Inflammatory Phenotype in Early Atherosclerosis
}

\author{
Elham Khanicheh ${ }^{1}$, Martina Mitterhuber ${ }^{1}$, Lifen $\mathrm{Xu}^{1}$, Stéphanie P. Haeuselmann ${ }^{1}$, Gabriela M. Kuster ${ }^{1,2}$, \\ Beat A. Kaufmann ${ }^{1,2 *}$
}

1 Department of Biomedicine, University of Basel and University Hospital Basel, Basel, Switzerland, 2 Cardiology, University Hospital Basel, Basel, Switzerland

\begin{abstract}
Background/Objectives: Inflammatory changes on the endothelium are responsible for leukocyte recruitment to plaques in atherosclerosis. Noninvasive assessment of treatment-effects on endothelial inflammation may be of use for managing medical therapy and developing novel therapies. We hypothesized that molecular imaging of vascular cell adhesion molecule-1 (VCAM-1) with contrast enhanced ultrasound (CEU) could assess treatment effects on endothelial phenotype in early atherosclerosis.

Methods: Mice with atherosclerosis produced by gene deletion of the LDL-receptor and Apobec-1-editing protein were studied. At 12 weeks of age, mice received 8 weeks of regular chow or atorvastatin-enriched chow $(10 \mathrm{mg} / \mathrm{kg} / \mathrm{day})$. At 20 weeks, CEU molecular imaging for aortic endothelial VCAM-1 expression was performed with VCAM-1-targeted (MB and control microbubbles $\left(\mathrm{MB}_{\mathrm{Ctr}}\right)$. Aortic wall thickness was assessed with high frequency ultrasound. Histology, immunohistology and Western blot were used to assess plaque burden and VCAM-1 expression.

Results: Plaque burden was reduced on histology, and VCAM-1 was reduced on Western blot by atorvastatin, which corresponded to less endothelial expression of VCAM-1 on immunohistology. High frequency ultrasound did not detect differences in aortic wall thickness between groups. In contrast, CEU molecular imaging demonstrated selective signal enhancement for $M_{V} B_{V A M}$ in non-treated animals ( $M_{V C A M} 2 \pm 0.3$ vs $M B_{C t r} 0.7 \pm 0.2, p<0.01$ ), but not in statin-treated animals ( $\left(\mathrm{MB}_{\mathrm{VCAM}} 0.8 \pm 0.2\right.$ vs $\mathrm{MB}_{\mathrm{Ctr}} 1.0 \pm 0.2, \mathrm{p}=\mathrm{ns} ; \mathrm{p}<0.01$ for the effect of statin on $\mathrm{MB}_{\mathrm{VCAM}}$ signal).

Conclusions: Non-invasive CEU molecular imaging detects the effects of anti-inflammatory treatment on endothelial inflammation in early atherosclerosis. This easily accessible, low-cost technique may be useful in assessing treatment effects in preclinical research and in patients.

Citation: Khanicheh E, Mitterhuber M, Xu L, Haeuselmann SP, Kuster GM, et al. (2013) Noninvasive Ultrasound Molecular Imaging of the Effect of Statins on Endothelial Inflammatory Phenotype in Early Atherosclerosis. PLoS ONE 8(3): e58761. doi:10.1371/journal.pone.0058761

Editor: Gerard Pasterkamp, University Medical Center Utrecht, Netherlands

Received January 3, 2013; Accepted February 6, 2013; Published March 15, 2013

Copyright: @ 2013 Khanicheh et al. This is an open-access article distributed under the terms of the Creative Commons Attribution License, which permits unrestricted use, distribution, and reproduction in any medium, provided the original author and source are credited.

Funding: This study was supported by SCORE grants from the Swiss National Science Foundation to Dr. Kaufmann (SNSF 32323B_123819 and 3232B0-141603) and to Dr. Kuster (SNSF 3232B-111352 and 3200B-111353). Dr. Khanicheh is supported by an MD-PhD start up grant from the University Hospital Basel. The funders had no role in study design, data collection and analysis, decision to publish, or preparation of the manuscript.

Competing Interests: The authors have declared that no competing interests exist.

*E-mail: kaufmannb@uhbs.ch
\end{abstract}

\section{Introduction}

Large primary and secondary prevention trials have consistently shown a risk reduction for cardiovascular events when patients with established vascular disease, diabetes or hyperlipidemia are treated with statins [1-3]. In addition, a specific subset of patients with low LDL cholesterol levels and elevated high sensitive Creactive protein also benefits from statin treatment [4]. However, statin treatment confers a risk reduction for cardiovascular events of $40 \%$ at best [5], thus leaving the majority of events to occur despite treatment. This residual risk may be attributable to incomplete reduction in inflammatory status despite reaching LDL-goals. Improved strategies for atherosclerosis treatment will likely include better risk assessment tools that allow for risk stratification and treatment during the early stages of atherosclerosis, and additional pharmacologic interventions that target inflammation. For pharmacologic interventions that are started at early timepoints during the pathogenesis of atherosclerosis, or that include novel, costly drug regimens with the goal of reducing vascular inflammation, the ability to non-invasively assess treatment effect on vascular inflammatory status will therefore be important.

Endothelial expression of the inflammatory cell adhesion molecule VCAM-1 plays an important role in the pathogenesis of atherosclerosis by regulating leukocyte recruitment to nascent atherosclerotic plaques in the arterial wall [6], and assessment of VCAM-1 expression may be a good target for testing the adequacy of anti-inflammatory treatment effects both for preclinical research in animal models, but also in humans. Ultrasound molecular imaging is a low-cost, easily accessible screening method that has been shown to be feasible for the assessment of VCAM-1 
expression during early atherosclerosis [7], and thus may allow for assessment of the adequacy of anti-inflammatory treatment in early atherosclerosis.

In the present study, we therefore investigated whether in vivo ultrasound molecular imaging of the expression of VCAM-1 could be used to assess the effects of pharmacologic therapy on vascular endothelial phenotype in a mouse model of early atherosclerosis. To examine how molecular imaging of the endothelial phenotype compares to assessment of vessel wall morphology, high frequency ultrasound imaging of the aortic arch was also performed.

\section{Methods}

\section{Mouse Model}

All experiments were performed in accordance with Swiss Federal Legislation and were approved by the local Animal Care and Use Committee of the University Hospital of Basel and the ethics committee of the Veterinary Office of the Canton of Basel. Mice with a double knockout for the LDL receptor and the Apobec-1 editing enzyme on a C57Bl/6 background were used. These mice develop atherosclerosis in a predictable, time dependent fashion while on a normal chow diet [8]. At 12 weeks of age, the mice were put either on a chow diet containing $0.01 \%$ $(\mathrm{wt} / \mathrm{wt}$ ) atorvastatin, resulting in a dose of approximately $10 \mathrm{mg} /$ $\mathrm{kg} /$ day $(\mathrm{n}=19)$ or an identical chow diet without atorvastatin $(n=18)$. At 20-22 weeks of age, mice were anesthetized with inhaled isoflurane (1 to $2 \%$ ), and a jugular intravenous catheter was placed for administration of microbubbles. The adequacy of anesthesia was regularly assessed with toe-pinch, and the dose of inhaled isoflurane adjusted if necessary. High frequency ultrasound and ultrasound molecular imaging for the expression of VCAM-1 were performed. Subsequently, blood from randomly selected animals $(n=7)$ from the statin group, $n=6$ from the nontreated group) was collected into EDTA-coated tubes by puncture of the left ventricle and was centrifuged at $4^{\circ} \mathrm{C}$ for $20 \mathrm{~min}$ at $2800 \mathrm{rpm}$ to obtain serum. Total cholesterol, combined low density lipoprotein (LDL) and very low density lipoprotein (VLDL) cholesterol as well as high density lipoprotein (HDL) cholesterol were measured using a commercial assay kit (EHDL-100, BioAssay-Systems, Hayward, CA). Animals were sacrificed by cervical dislocation under deep sedation. The aortas were harvested for histology, fluorescent immunohistochemistry, and VCAM-1 western blotting.

\section{Microbubble Preparation}

Biotinylated, lipid-shelled decafluorobutane microbubbles were prepared by sonication of a gas saturated aqueous suspension of distearoylphosphatidylcholine $(2 \mathrm{mg} / \mathrm{ml}$; Avanti Polar Lipids, Alabaster AL), polyoxyethylene-40-stearate $(1 \mathrm{mg} / \mathrm{ml}$; Sigma), and 1,2-distearoyl-sn-glycero-phosphoethanolamine-N-[biotinyl(polyethylene glycol)-3400] $(0.15 \mathrm{mg} / \mathrm{ml}$, Creative PEG Works,

Table 1. Effect of statin treatment on serum lipid profile (mg/ dl) in LDL-/-Apobec $1-/-$ mice ( $n=7$ statin group, $n=6$ non-treated group).

\begin{tabular}{llll}
\hline & & & \\
\hline & Non-treated & Statin & P- value \\
\hline Total cholestrol & $290.5 \pm 11.5$ & $261.5 \pm 19.4$ & ns \\
LDL+VLDL & $233 \pm 6.7$ & $189.1 \pm 18.2$ & 0.03 \\
HDL & $53.8 \pm 3.8$ & $48.2 \pm 3.9$ & ns \\
\hline
\end{tabular}

doi:10.1371/journal.pone.0058761.t001
Winston Salem, NC). Microbubbles targeted to VCAM-1 $\left(\mathrm{MB}_{\mathrm{VCAM}}\right)$ were prepared by conjugation of biotinylated rat anti-mouse VCAM-1 antibody (MK 2.7) to the microbubble surface using biotin-streptavidin-biotin linking as previously described [9]. Control microbubbles $\left(\mathrm{MB}_{\mathrm{Ctr}}\right)$ bearing a non-specific isotype control antibody (R3-34, BD Bioscience) were also prepared. Microbubble concentration and size were measured by electrozone sensing (Multisizer III, Beckman- Coulter). There was no size difference between $\mathrm{MB}_{\mathrm{VCAM}-1}$ and $\mathrm{MB}_{\mathrm{C} \text { tr }}(3.2 \mu \mathrm{m}$ \pm 0.1 vs. $3.1 \mu \mathrm{m} \pm 0.1, p=n s)$.

\section{Contrast Enhanced Ultrasound Molecular Imaging}

Ultrasound imaging (Sequoia Acuson C512; Siemens Medical Systems USA Inc., Mountain View, CA) was performed with a high-frequency linear-array probe (15L8) held in place by a railed gantry system. The ascending aorta of the mouse was imaged in a long axis plane from a right parasternal window, care was taken to include the sinus of valsalva and the take-off of the brachiocephalic artery in the image. Contrast enhanced ultrasound (CEU) was performed with power modulation and pulse inversion (Contrast Pulse Sequence) imaging at a centerline frequency of $7 \mathrm{MHz}$ and a dynamic range of $50 \mathrm{~dB}$. The gain settings were adjusted to levels just below visible noise speckle and held constant. $\mathrm{MB}_{\mathrm{VCAM}}$ or $\mathrm{MB}_{\mathrm{Ctr}}\left(1 \times 10^{6}\right.$ microbubbles per injection) were injected intravenously via a cannulated jugular vein in random order, while ultrasound imaging was paused. Eight minutes after microbubble injection, imaging was resumed at a mechanical index of 0.87 . The first acquired image frame was used to derive the total amount of microbubbles present within the aorta. The microbubbles in the ultrasound beam were then destroyed with several $(>10)$ image frames. Several image frames at a long pulsing interval $(10 \mathrm{sec})$ were then acquired to measure signal attributable to freely circulating microbubbles. Data were log-linear converted, and frames representing freely circulating microbubbles were digitally subtracted from the first image to derive signal from attached microbubbles alone. Contrast intensity was measured from a region of interest encompassing the sinus of valsalva, the ascending aorta and the initial portion of the aortic arch, extending into the origin of the brachiocephalic artery. The selection of the region of interest was guided by fundamental frequency anatomic images of the ascending aorta acquired at $14 \mathrm{MHz}$ at the end of each individual imaging sequence.

\section{High Frequency Ultrasound Assessment of Plaque Size and Cardiac Function}

High frequency imaging of the aortic arch in long axis was performed with a Vevo 2100 (Visual Sonics Inc.) imaging system equipped with an MS550D transducer operating at $40 \mathrm{MHz}$ at the shortest possible pulse length. This system has an axial resolution of $40 \mu \mathrm{m}$ [10]. Plaque size was assessed by measuring the vessel wall thickness along the axial orientation of the ultrasound beam on real time images at the sinus of valsalva, the greater curvature, the lesser curvature, and at the take off of the brachiocephalic artery by an investigator blinded to animal treatment. M-Mode images of the left ventricle at the height of the papillary muscles were used to calculate fractional shortening, aortic peak flow velocity was measured on pulsed wave spectral doppler tracings from the aortic arch.

\section{Histology}

All histological preparations were done on sections from the base of the aorta at the sinus of valsalva and from the ascending aorta at the take off of the brachiocephalic artery. Due to the 
A
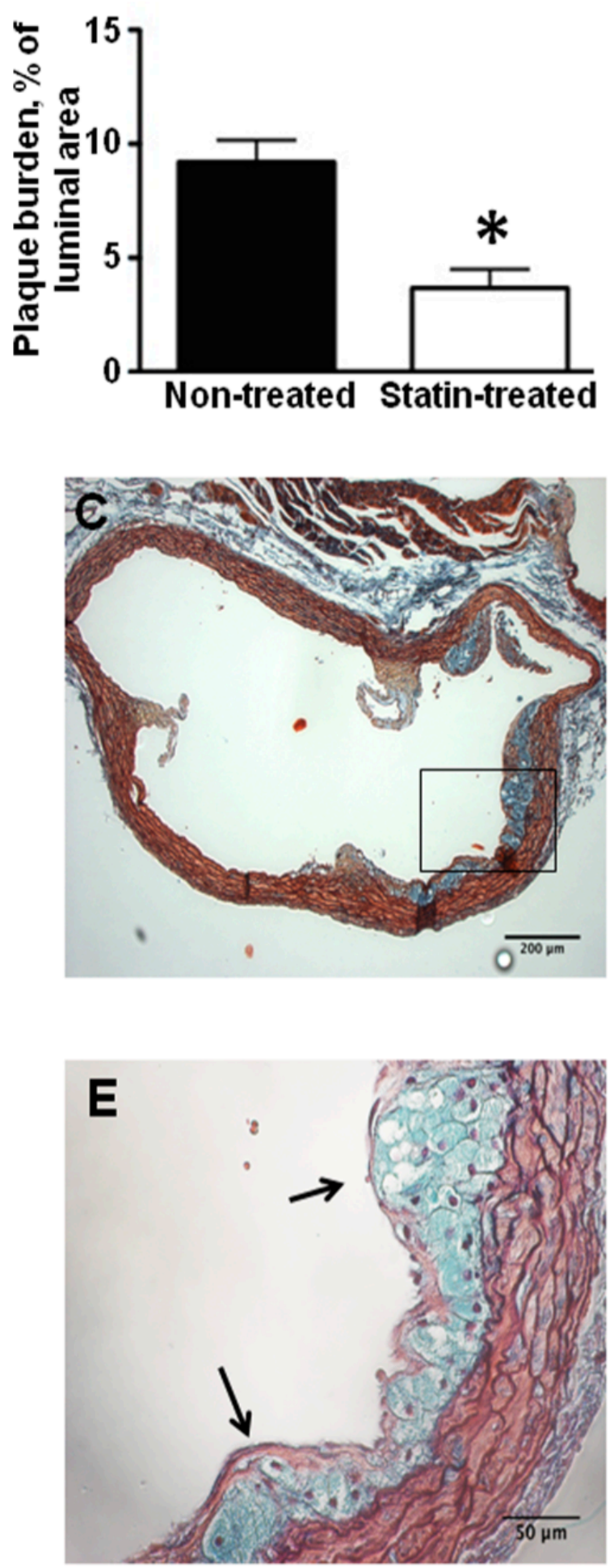

B
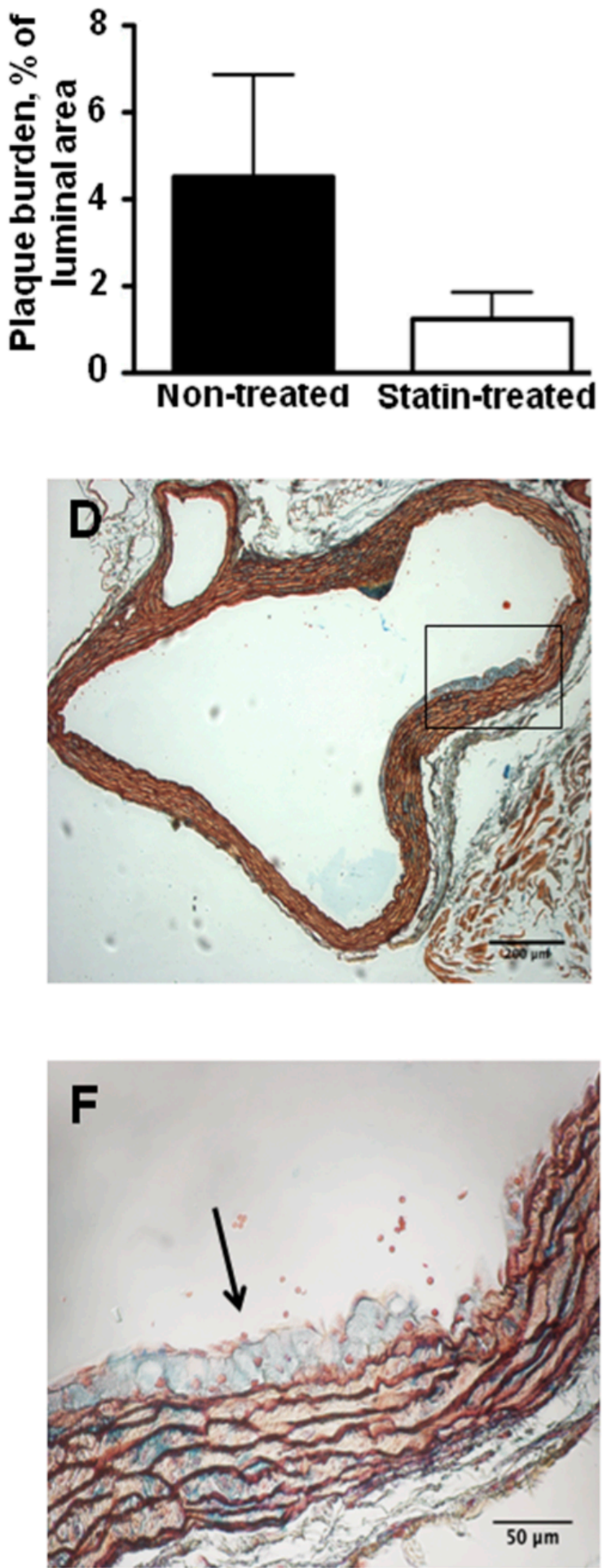

Figure 1. Effect of statin treatment on aortic atherosclerotic plaque burden. Percent of total luminal plaque area at the base of aorta (A) and in the ascending aorta (B) in non-treated animals versus statin treated animals $(\mathbf{A}, n=11$ non-treated animals, $n=7$ statin treated animals; $\mathbf{B}, n=7$ non-treated and $n=7$ statin treated animals), ${ }^{*} p<0.05$ vs non-treated animals. Examples of Movat's pentachrom stains at the base of aorta in a non- 
treated mouse $(\mathbf{C}, \mathbf{E})$ and a statin treated mouse $(\mathbf{D}, \mathbf{F})$. The arrows denote a large plaque in a magnified view from a non-treated animal $(\mathbf{E})$ and a small plaque in a magnified view from a statin treated animal (F). doi:10.1371/journal.pone.0058761.g001

limited amount of tissue available and different embedding techniques, it was not possible to perform every histological assessment in every animal. For assessment of plaque burden, Movat's pentachrome staining was performed. Paraffin-embedded aortas were sectioned in short axis from the aortic valve to the distal portion of the ascending aorta at the take off of the brachiocephalic artery and stained using a commercially available Movat's pentachrome staining kit (F-384, Rowley Biochemical Inc). Histological plaque burden was measured as the plaque area in relation to the vessel area defined by the internal elastic lamina using Image-J.

Fluorescent immunohistochemistry was performed to qualitatively assess the endothelial expression of VCAM-1. Frozen aortic sections were mounted on glass slides, fixed in $-20^{\circ} \mathrm{C}$ Acetone, air-dried, blocked with $10 \%$ goat serum in TBS/FSGO and incubated overnight at $4^{\circ} \mathrm{C}$ with anti-VCAM-1 (CBL-1300, Millipore). Goat anti-rat Alexa-633 (A21094, Invitrogen) was added and incubated for 1 hour at room temperature. Sections were mounted with Prolong gold antifade mounting medium and imaged on a Zeiss LSM 710 confocal microscope.

Immunostaining for Mac-2 was performed on paraffin-embedded aortic sections after heat-induced antigen retrieval with $10 \mathrm{mM}$ NaCitrate Buffer $(\mathrm{pH}=6)$. Sections were blocked with $3 \%$ BSA in PBS and incubated overnight at $4^{\circ} \mathrm{C}$ with anti-Mac-2 (ACL8942AP, lucerna-chem). Goat anti-rat Alexa-633 (A21094, Invitrogen) was added and incubated for 1 hour at room temperature. Sections were mounted with Prolong gold antifade mounting medium containing DAPI (P36935, Invitrogen) and imaged on a Zeiss LSM 710 confocal microscope. For quantitative immunocytochemical comparisons of macrophage content, the number of positively stained pixels was counted on thresholded pictures and normalized to total number of pixels of the vessel area encompassed by the internal elastic lamina using Image-J. Thresholds were defined as the mean background intensity plus 40 times the standard deviation in each individual picture. For each mouse at least two sections on different slides were imaged and quantified for both the base and ascending aorta.

\section{Western Blotting}

Western blotting for the expression of VCAM-1 was performed in 5 statin treated animals and 5 non-treated animals that had not undergone ultrasound imaging. The ascending portion of the aorta was homogenized in lysis buffer (Cell Signaling) containing $80 \mathrm{mmol} / \mathrm{L}$ Pefabloc SC plus (Roche). Protein concentration was measured using the Micro BCA (bicinchoninic acid) protein assay kit (Thermo Scientific). $10 \mu \mathrm{g}$ of protein were resolved on SDSPAGE and transferred to Polyvinylidene fluoride (PVDF) membranes (Amersham). Membranes were probed with monoclonal rat anti-mouse VCAM-1 (Clone \# 112702, R\&D Systems) and monoclonal anti- $\boldsymbol{\alpha}$-tubulin (Clone DM1A, Sigma) antibodies. Blots were subsequently incubated with horseradish peroxidaseconjugated secondary antibodies (Jackson Immuno Research) and
A
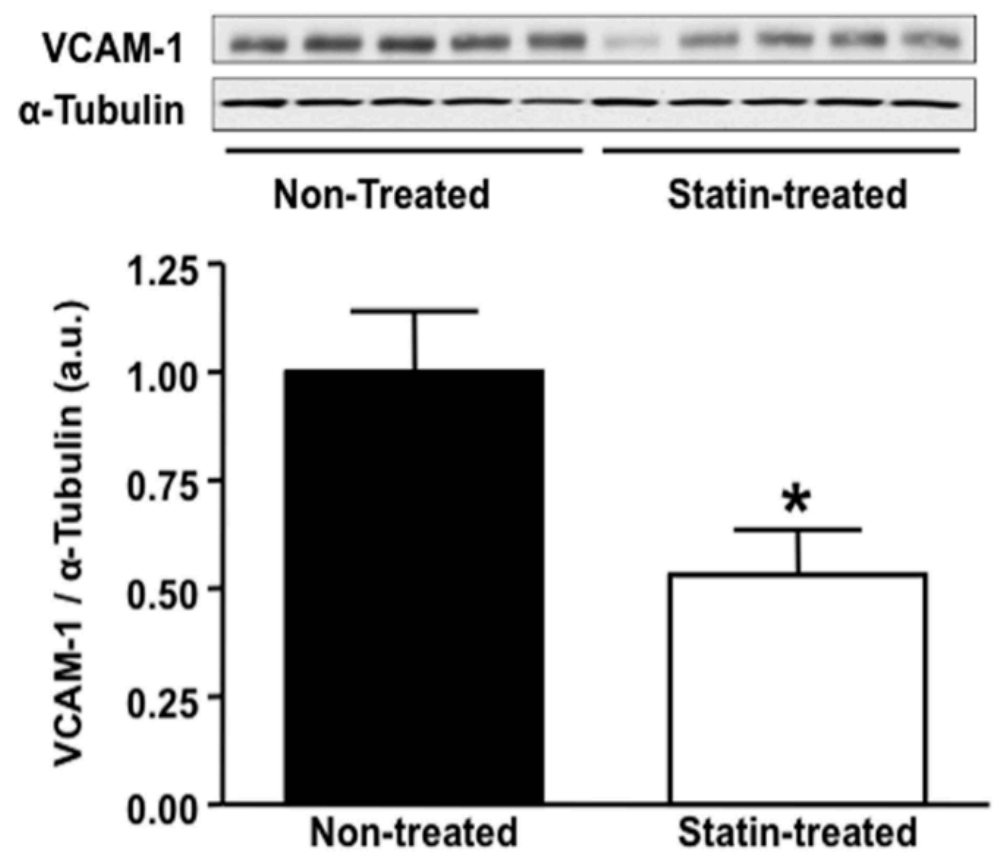
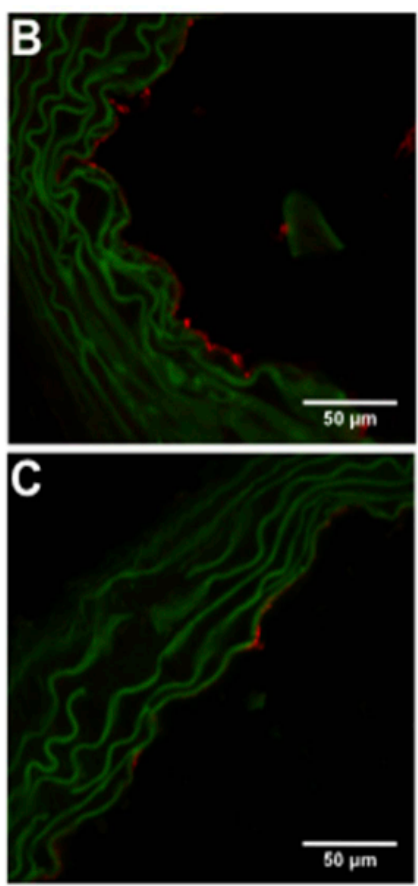

Figure 2. Effect of statin treatment on aortic expression of VCAM-1. (A) VCAM-1 protein expression in the ascending aorta assessed by Western blot in non-treated (lanes 1-5) versus statin treated (lanes $6-10$ ) animals, $n=5$ per group, ${ }^{*} p<0.01$ vs non-treated animals. Representative examples of fluorescent immunohistochemistry images of the base of the aorta demonstrating endothelial VCAM-1 expression (red fluorescence) in a non-treated animal (B) and a statin treated animal (C), the green fluorescence is autofluorescence delineating vessel anatomy. doi:10.1371/journal.pone.0058761.g002 
A

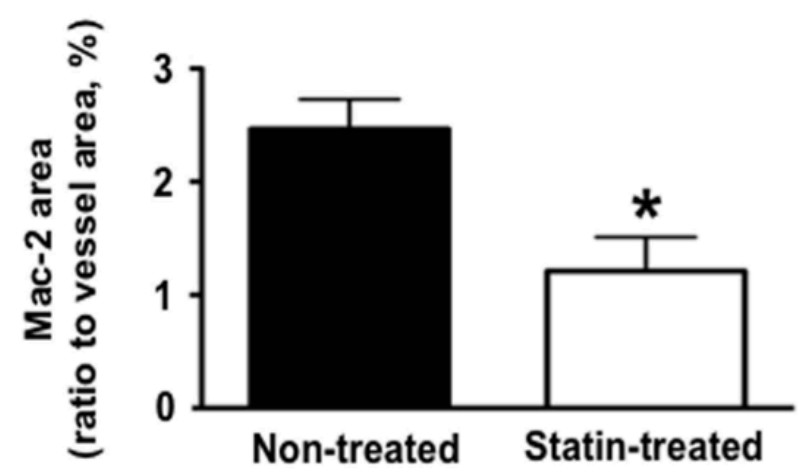

B

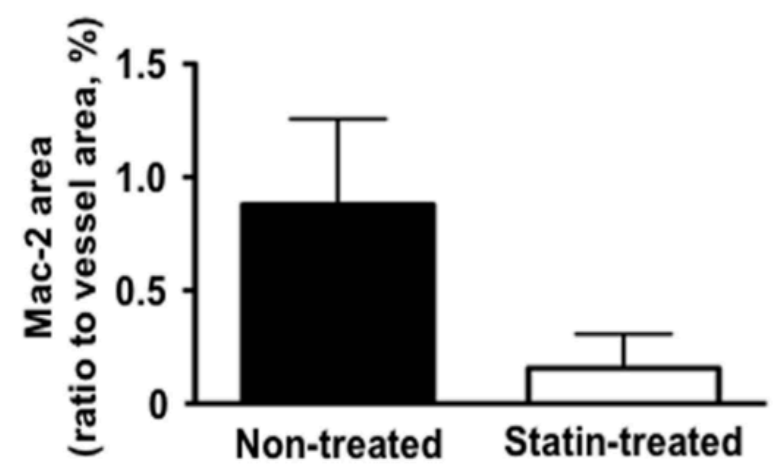

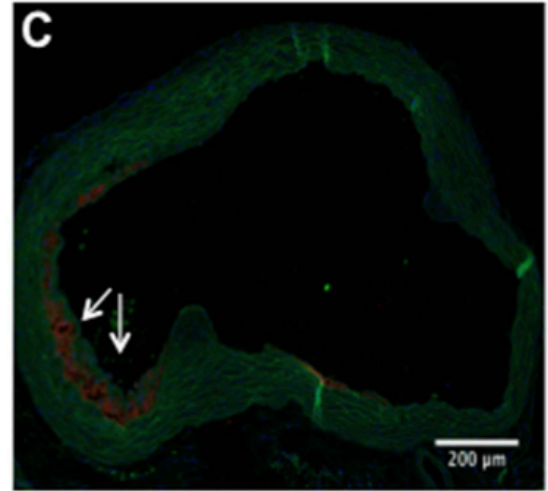

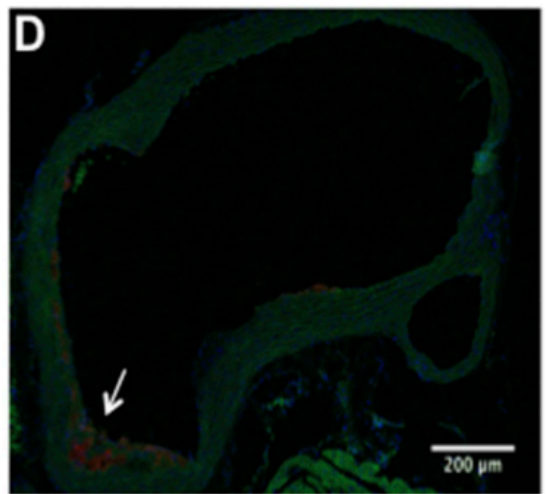

Figure 3. Effect of statin treatment on vascular inflammation. Fluorescent immunohistochemistry for the expression of macrophage Mac- 2 at the base of the aorta $(\mathbf{A})$ and in the ascending aorta $(\mathbf{B})$ of non-treated versus statin treated mice $(\mathbf{A}, n=12$ non-treated animals, $n=9$ statin treated animals; B, $n=8$ non-treated and $n=8$ statin treated animals), ${ }^{*} p<0.05$ versus non-treated animals. Examples of Mac- 2 staining in a non-treated mouse (C) and a statin treated mouse (D), arrows denote stained Mac-2 positive macrophages. doi:10.1371/journal.pone.0058761.g003

band intensities were detected by enhanced chemiluminescence (Western Lightening Plus; Perkin Elmer) and quantitated using NIH ImageJ software (http://rsbweb.nih.gov/ij/).

\section{Statistical Analysis}

Data were analyzed on GraphPad Prism (version 5.0d). Data are expressed as mean \pm SEM unless stated otherwise. Single comparisons between the two animal groups were performed with a Mann Whitney test. Friedman's repeated measures ANOVA with Dunn's post hoc test was used to compare differences in microbubble signals within and between animal groups. A p value $<0.05$ (2-sided) was considered statistically significant.

\section{Results}

Effect of Statin Treatment on Cholesterol Levels

Statin treatment did not significantly influence total cholesterol or HDL-cholesterol levels, despite a trend towards decreased levels for both (table 1). In contrast, statin treatment resulted in a modest, but statistically significant $20 \%$ reduction in VLDL+LDL cholesterol levels.

\section{Histologic Plaque Area}

Movat's Pentachrome stains in non-treated animals showed macrophage-rich type III atherosclerotic lesions in the sinus of valsalva that occupied close to $8 \%$ of the vessel lumen area. Statin treatment resulted in a significant reduction in plaque burden in the sinuses of valsalva. In the ascending aorta, there was a similar, albeit statistically nonsignificant reduction in plaque area in animals that were receiving statin treatment (Figure 1).

\section{Expression of VCAM-1 and Vascular Inflammation}

Expression of VCAM-1 on Western blot was reduced by $47 \%$ (Figure 2A) in statin-treated animals. On immunohistochemistry, VCAM-1 staining was intense on endothelial cells both in plaque areas, but also on endothelium overlying apparently normal wall areas, and on macrophages inside plaques in non-treated animals. In contrast, endothelial staining was less pronounced on the endothelium in statin treated animals (Figure 2B and Figure 2C). Statin treatment resulted not just in a decrease in plaque area, but also in reduced macrophage content of plaques as assessed by Mac-2 staining (Figure 3A and 3B).

High Frequency Ultrasound Assessment of Plaque Size and Cardiac Function

The aortic wall thickness was measured at atherosclerosis-prone sites throughout the ascending aorta. Despite histological differences in vessel wall thickness $(59 \pm 22 \mu \mathrm{m})$ between statin treated and non-treated animals in plaque areas in the sinuses of valsalva that were above the theoretical axial resolution of the ultrasound 
A

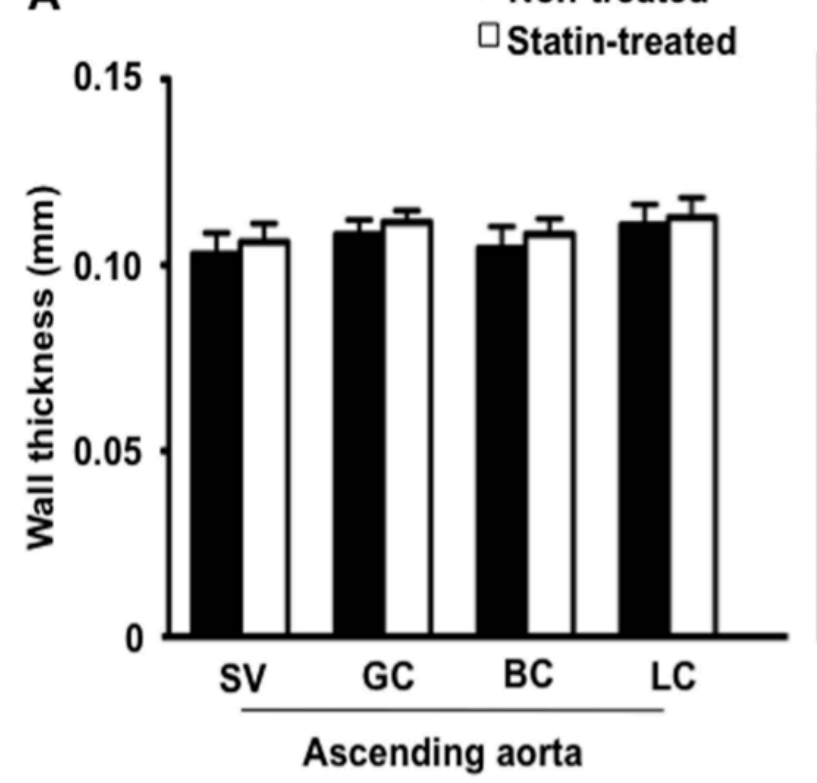

B

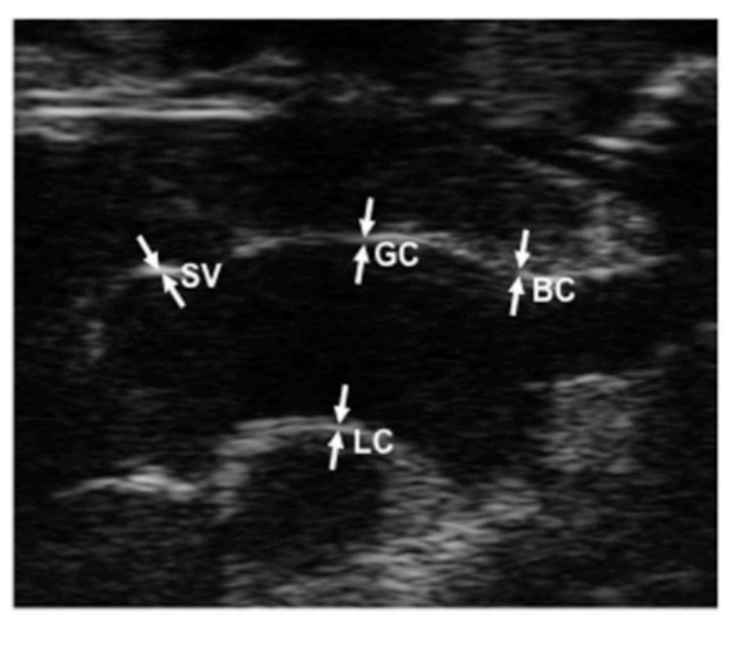

Figure 4. High frequency ultrasound imaging ( $40 \mathrm{MHZ}$ ) of the ascending aorta. (A) Wall thickness of the ascending aorta measured at the sinus of valsalva (SV), the greater curvature (GC), the lesser curvature (LC) and at the origin of brachoicephalic artery (BC) in non-treated and statin treated animals ( $n=10$ per group). (B) Example of high frequency ultrasound imaging of the ascending aorta illustrating the measurements obtained. doi:10.1371/journal.pone.0058761.g004

system, blinded assessment of vessel wall thicknessess did not differ between the treatment groups at any of the measured points (Figure 4).

Left ventricular fractional shortening, aortic diameter, and aortic peak flow velocity did not systematically differ between statin treated and non-treated animals, indicating that shear rate conditions that oppose selective microbubble attachment in the ascending aorta did not differ between animal groups (Table 2).

\section{Molecular Imaging of VCAM-1}

CEU molecular imaging showed selective signal enhancement for microbubbles targeted to VCAM-1 in non-treated animals, with a $120 \%$ signal increase relative to signal from control microbubbles in the same animal group. In contrast, signal for microbubbles targeted to VCAM-1 was not increased over control microbubble signal in animals on statin treatment (Figure 5).

Given the differences in plaque burden between the sinus of valsalva and the distal ascending aorta on histology, in an exploratory analysis, we also assessed the regional effect of statin treatment on CEU molecular imaging signal for microbubbles targeted to VCAM-1. In non-treated animals, signal for VCAM-1 targeted microbubbles was slightly, but not significantly lower in

\begin{tabular}{|c|c|c|c|}
\hline & Non-treated & Statin & P- value \\
\hline Fractional shortening (\%) & $35.0 \pm 2.7$ & $36.8 \pm 3.1$ & ns \\
\hline Aortic Internal Diameter (mm) & $1.3 \pm 0.1$ & $1.3 \pm 0.1$ & ns \\
\hline Aortic Peak Systolic Velocity (m/s) & $0.63 \pm 0.15$ & $0.61 \pm 0.10$ & ns \\
\hline
\end{tabular}

the distal ascending aorta compared to the base of the aorta. In both regions of interest, statin treatment resulted in a similar reduction in signal with significantly lower signals than in animals in non-treated animals (Figure 6).

\section{Discussion}

Current treatment of atherosclerosis is to a large extent directed at patients with established clinical disease. However, it can be anticipated that in the future existing and emerging therapeutic agents will be employed at earlier stages during the disease process with the goal of quelling the molecular pathways that lead to the buildup of plaques, including endothelial recruitment of inflammatory cells. Such strategies will also create the need for a method for early, noninvasive detection of treatment effects. The results from our study indicate that ultrasound molecular imaging can detect the impact of therapies aimed at reducing endothelial inflammation during early disease stages in a murine model of atherosclerosis when high frequency morphologic plaque imaging is unable to detect a treatment effect.

The mouse model of atherosclerosis that we studied is characterized by a progressive development of atherosclerotic plaques with the first minimal lesions detected around 10 weeks of age, when treatment was started in our study. At 20 weeks of age, the mouse model shows lesions that cover about $5 \%$ of the total aortic surface, but $25-30 \%$ of the proximal aorta [7] in nontreated animals. The histologic changes found in early atherosclerosis such as in our mouse model are characterized by the accumulation of macrophages in the intima of the arterial wall. Recruitment of monocytes from the blood stream is tightly regulated by cell adhesion molecules expressed on the vascular endothelium, and VCAM-1 is essential for the development of early atherosclerotic lesions [11]. VCAM-1 is expressed on endothelial cells during the earliest stages of atherosclerosis [12], 

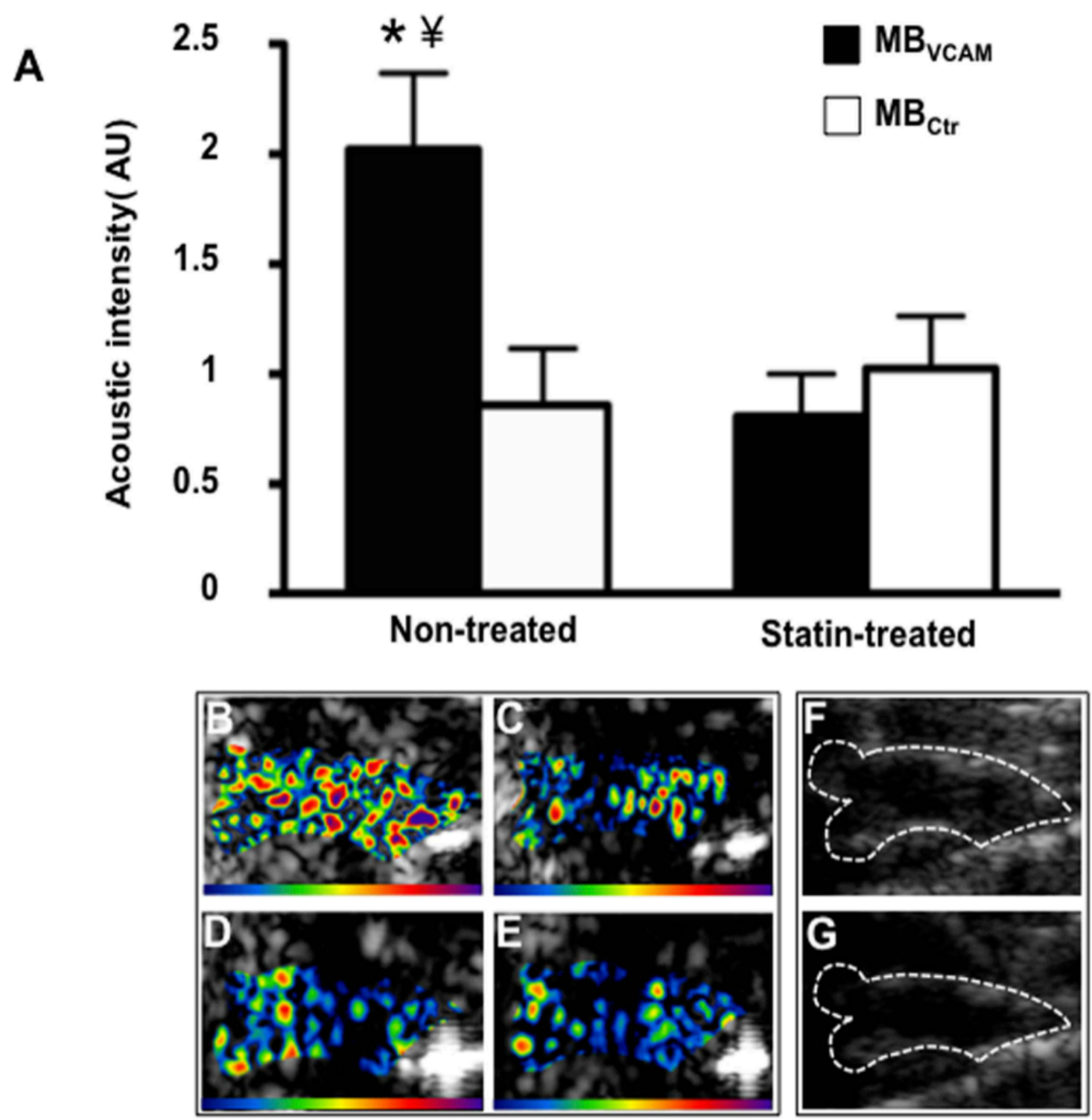

Figure 5. Molecular imaging of the ascending aorta. (A) Mean \pm SEM background-subtracted signal intensity for microbubbles targeted to VCAM-1 (MB $\left.B_{\text {CAM }}\right)$ and control microbubbles $\left(M B_{C r}\right)$ in non-treated $(n=10)$ and statin treated animals $(n=12) .{ }^{*} p<0.01$ vs $M B$ in non-treated animals, $¥ p<0.01$ vs MBVCAM in statin treated animals. Examples of color coded CEU images from a non-treated animal after injection of $M B_{V C A M}(B)$, and of $M_{C_{C t r}}(C)$. Images from a statin treated animal after injection of $M_{\mathrm{VCAM}}(\mathbf{D})$, and of $\mathrm{MB}_{\mathrm{Ctr}}(\mathbf{E})$. The color scale for the CEU images is shown at the bottom of each frame. (F) and (G) illustrate the outline of the ascending aorta on B-mode ultrasound images which was used as a region of interest for acoustic intensity measurements. doi:10.1371/journal.pone.0058761.g005

and has been shown to be an ideal target for ultrasound molecular imaging of vascular inflammation in both early and established atherosclerosis [7,13]. Statin treatment reduces vascular inflammation and the expression of VCAM-1 on the vascular endothelium. This effect is mediated through a reduction in LDL-cholesterol [14], but also independent of the effects on serum cholesterol levels [15]. VCAM-1 is not stored in endothelial cells, and its expression on vascular endothelial cells depends on de novo synthesis of the protein. Regulation of VCAM-1 gene transcription involves the activation of $\mathrm{NF}-\mathrm{kB}$ as a response to inflammatory stimuli. Statin treatment has been shown to reduce the activation
NF-kB in endothelial cells [16] and thus attenuate the transcription of VCAM-1 mRNA.

The effect of statins in different mouse models of atherosclerosis has been variable [17]. In the mouse model that we used in our study, statin treatment resulted in a modest reduction in serum cholesterol levels. However, we found a more pronounced slowing of the progression of atherosclerotic plaque development, a reduction in VCAM-1 expression and reduced vascular inflammation, consistent with the aforementioned pleiotropic effects of statins on inflammatory pathways. 


\section{A}
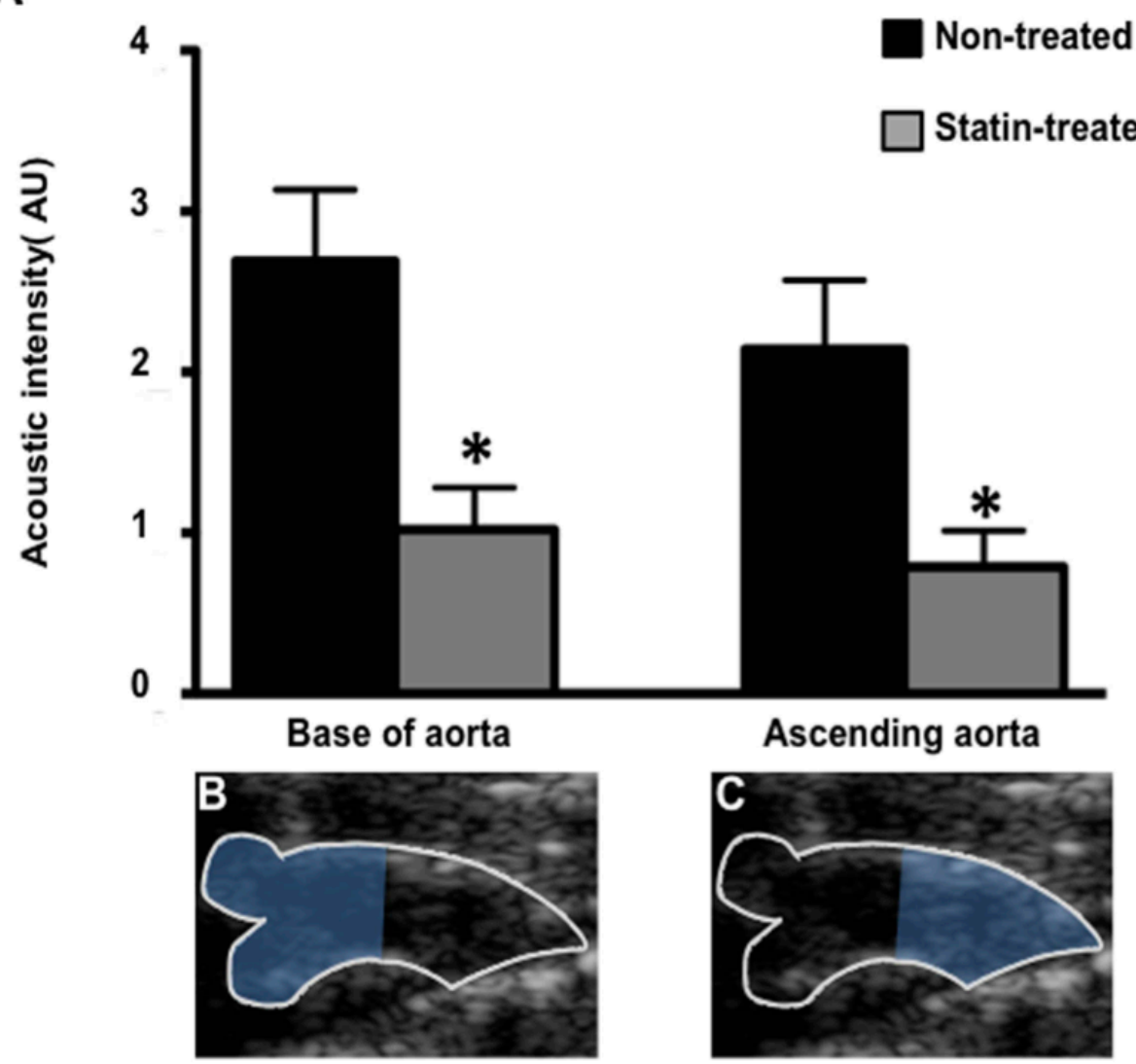

Figure 6. CEU molecular imaging of the regional endothelial expression of VCAM-1. (A) Mean \pm SEM background-subtracted signal intensity for microbubbles targeted to VCAM-1 at the base of the aorta and in the distal ascending aorta in non-treated ( $n=9)$ and statin treated animals $(n=10) * p<0.05$ vs non-treated animals. Two dimensional ultrasound imaging illustrating outline of $(\mathbf{B})$ base of the aorta and $(\mathbf{C})$ ascending aorta.

doi:10.1371/journal.pone.0058761.g006

It has previously been shown that molecular imaging with targeted MRI and SPECT probes can be used for assessing the effect of statins in animal models of advanced atherosclerosis $[18,19]$. Our study is the first to show that ultrasound molecular imaging is capable of specifically detecting the effects of pharmacologic treatment on endothelial inflammatory phenotype during the very early stages of the pathogenesis of atherosclerosis. It should be noted that, as opposed to molecular imaging performed with MRI and SPECT that relies on tracer retention not only on the endothelium but also within plaques, ultrasound molecular imaging specifically detects tracer retention on the vascular endothelial surface. Given the important role that endothelial expression of cell adhesion molecules has during the initial pathogenesis of atherosclerosis, an imaging approach that is capable of assessing endothelial phenotypic changes with high sensitivity is well suited not only for early detection [7] but also for assessing treatment effects during the initial stages of atherosclerosis. Similar to ultrasound carotid intima media thickening that has been used for risk assessment in clinical studies, we also performed high resolution ultrasound imaging with the intent to measure the effect of statin therapy on aortic wall thickness. Despite differences in plaque thickness on histology that were in the range of the theoretical axial resolution $(40 \mu \mathrm{m})$ of the ultrasound system, no differences in wall thickness were detected on ultrasound imaging. In contrast, ultrasound molecular imaging was able to detect the effect of statin treatment on endothelial inflammatory phenotype. This was true for both the proximal ascending aorta, but also for the distal ascending aorta, which on histology showed a lower plaque area. The lower signal for microbubbles targeted to VCAM-1 in statin treated animals was associated not only with a reduction in VCAM-1 expression, but also with a reduced vascular inflammation as evidenced by macrophage Mac-2 staining.

Several limitations of our study should be mentioned. We used high-dose statin treatment in our animal model, and did not evaluate whether ultrasound molecular imaging would be capable of detecting graded responses to several different doses of statin treatment. As we did not perform examinations at multiple timepoints, we cannot comment on whether changes in vascular inflammatory phenotype precede changes in histology. Further it has been shown that microbubble targeting efficiency not only depends on the expression of target molecules, but also on endothelial glycocalyx thickness [20], which has been shown to be perturbed in atherosclerotic vessels, and can be partially restored by statin therapy [21]. Therefore, the effect on VCAM-1 signal in statin treated animals might also partially be due to a reduced targeting efficiency of $\mathrm{MB}_{\mathrm{VCAM}}$ on the aortic endothelial surface. Due to the small size of the murine model of atherosclerosis in our 
study, the expression of VCAM-1 was imaged in the aorta. With regards to translation into humans, the aorta will not be a suitable vessel for imaging, nor will it be likely that noninvasive ultrasound molecular imaging of the coronary arteries will be feasible in the near future. However, the carotid artery is an accepted surrogate vessel for the assessment of cardiovascular risk and will be easily accessible for ultrasound molecular imaging in humans. Given the differences in plaque burden in the sinuses of valsalva versus the ascending aorta, we performed a regional analysis of VCAM-1 targetd signal. This analysis was not compared to regional assessment of VCAM-1 expression, in particular Western blot, and is to be viewed as an exploratory analysis. Last, from our study we cannot draw conclusions on whether the assessment of treatment effects early during the pathogenesis of atherosclerosis would be of value in improving disease management.

In conclusion, we show that ultrasound molecular imaging can detect the effects of statin therapy on early inflammatory processes

\section{References}

1. Shepherd J, Cobbe SM, Ford I, Isles CG, Lorimer AR et al. (1995) Prevention of coronary heart disease with pravastatin in men with hypercholesterolemia. West of scotland coronary prevention study group. N Engl J Med 333: 1301-1307.

2. Downs JR, Clearfield M, Weis S, Whitney E, Shapiro DR et al. (1998) Primary prevention of acute coronary events with lovastatin in men and women with average cholesterol levels: Results of afcaps/texcaps. Air force/texas coronary atherosclerosis prevention study. JAMA 279: 1615-1622.

3. Sever PS, Dahlof B, Poulter NR, Wedel H, Beevers G et al. (2003) Prevention of coronary and stroke events with atorvastatin in hypertensive patients who have average or lower-than-average cholesterol concentrations, in the angloscandinavian cardiac outcomes trial-lipid lowering arm (ascot-lla): A multicentre randomised controlled trial. Lancet 361: 1149-1158.

4. Ridker PM, Danielson E, Fonseca FA, Genest J, Gotto AM et al. (2008) Rosuvastatin to prevent vascular events in men and women with elevated creactive protein. N Engl J Med 359: 2195-2207.

5. Chyu KY, Shah PK (2011) Emerging therapies for atherosclerosis prevention and management. Cardiol Clin 29: 123-135.

6. Ramos CL, Huo Y, Jung U, Ghosh S, Manka DR et al. (1999) Direc demonstration of p-selectin- and vcam-1-dependent mononuclear cell rolling in early atherosclerotic lesions of apolipoprotein e-deficient mice. Circ Res 84: $1237-1244$.

7. Kaufmann BA, Carr CL, Belcik JT, Xie A, Kron B et al. (2010) Molecular imaging of the initial inflammatory response in atherosclerosis: Implications for early detection of disease. Arterioscler Thromb Vasc Biol 30: 54-59.

8. Powell-Braxton L, Veniant M, Latvala RD, Hirano KI, Won WB et al. (1998) A mouse model of human familial hypercholesterolemia: Markedly elevated low density lipoprotein cholesterol levels and severe atherosclerosis on a low-fat chow diet. Nat Med 4: 934-938.

9. Lindner JR, Song J, Christiansen J, Klibanov AL, Xu F et al. (2001) Ultrasound assessment of inflammation and renal tissue injury with microbubbles targeted to p-selectin. Circulation 104: 2107-2112.

10. Foster FS, Zhang MY, Zhou YQ Liu G, Mehi J et al. (2002) A new ultrasound instrument for in vivo microimaging of mice. Ultrasound Med Biol. 28: 1165 1172.

11. Cybulsky MI, Iiyama K, Li H, Zhu S, Chen M et al. (2001) A major role for vcam-1, but not icam-1, in early atherosclerosis. J Clin Invest 107: 1255-1262. in atherosclerosis at a timepoint when high-resolution imaging is unable to ascertain differences in plaque development. Such a lowcost, easily accesible imaging strategy could in the future be useful in the future for assessing the effect of drug treatment in individual patients, but also for screening of the effect of novel drug classes other than statins in the preclinical field.

\section{Acknowledgments}

The authors would like to thank Dr. Jonathan Lindner for his scientific input and critical review of this manuscript.

\section{Author Contributions}

Conceived and designed the experiments: EK GK BAK. Performed the experiments: EK MM LX SH BAK. Analyzed the data: EK LX SH BAK. Contributed reagents/materials/analysis tools: EK MM LX SH GK BAK. Wrote the paper: EK BAK.

12. Iiyama K, Hajra L, Iiyama M, Li H, DiChiara M et al. (1999) Patterns of vascular cell adhesion molecule- 1 and intercellular adhesion molecule- 1 expression in rabbit and mouse atherosclerotic lesions and at sites predisposed to lesion formation. Circ Res 85: 199-207.

13. Kaufmann BA, Sanders JM, Davis C, Xie A, Aldred P et al. (2007) Molecular imaging of inflammation in atherosclerosis with targeted ultrasound detection of vascular cell adhesion molecule-1. Circulation 116: 276-284.

14. Bustos C, Hernandez-Presa MA, Ortego M, Tunon J, Diaz C et al. (1998) Hmgcoa reductase inhibition by atorvastatin reduces neointimal inflammation in a rabbit model of atherosclerosis. J Am Coll Cardiol 32: 2057-2064.

15. Sukhova GK, Williams JK, Libby P (2002) Statins reduce inflammation in atheroma of nonhuman primates independent of effects on serum cholesterol. Arterioscler Thromb Vasc Biol 22: 1452-1458.

16. Rasmussen LM, Hansen PR, Nabipour MT, Olesen P, Kristiansen MT et al. (2001) Diverse effects of inhibition of 3-hydroxy-3-methylglutaryl-coa reductase on the expression of vcam-1 and e-selectin in endothelial cells. Biochem J 360: $363-370$.

17. Zadelaar S, Kleemann R, Verschuren L, de Vries-Van der Weij J, van der Hoorn J et al. (2007) Mouse models for atherosclerosis and pharmaceutical modifiers. Arterioscler Thromb Vasc Biol 27: 1706-1721

18. Nahrendorf M, Jaffer FA, Kelly KA, Sosnovik DE, Aikawa E et al. (2006) Noninvasive vascular cell adhesion molecule-1 imaging identifies inflammatory activation of cells in atherosclerosis. Circulation 114: 1504-1511.

19. Fujimoto S, Hartung D, Ohshima S, Edwards DS, Zhou J et al. (2008) Molecular imaging of matrix metalloproteinase in atherosclerotic lesions: Resolution with dietary modification and statin therapy. J Am Coll Cardiol 52: $1847-1857$.

20. Khanicheh E, Mitterhuber M, Kinslechner K, Xu L, Lindner JR et al. (2012) Factors affecting the endothelial retention of targeted microbubbles: Influence of microbubble shell design and cell surface projection of the endothelial target molecule. J Am Soc Echocardiogr 25: 460-466.

21. Meuwese MC, Mooij HL, Nieuwdorp M, van Lith B, Marck R et al. (2009) Partial recovery of the endothelial glycocalyx upon rosuvastatin therapy in patients with heterozygous familial hypercholesterolemia. J Lipid Res 50: 148153. 
3.3 Rapid reduction of endothelial activation in early- stage atherosclerosis with apocynin independent of anti- oxidative properties.

Elham Khanicheh MD, Yue Qi MD, Aris Xie BS, Martina Mitterhuber MSc, Lifen Xu PhD, Michika Mochizuki PhD, Youssef Daali PhD, Vincent Jaquet $\mathrm{PhD}$, Karl-Heinz Krause MD, Zaverio Ruggeri MD, Gabriela M. Kuster MD, Jonathan R. Lindner MD, Beat A. Kaufmann MD 


\section{RAPID REDUCTION OF ENDOTHELIAL ACTIVATION IN EARLY-STAGE ATHEROSCLEROSIS WITH APOCYNIN IS INDEPENDENT OF ANTI- OXIDATIVE PROPERTIES}

Elham Khanicheh, MD, Yue Qi, MD, Aris Xie, BS, Martina Mitterhuber, MSc, Lifen Xu, PhD, Michika Mochizuki PhD, Youssef Daali PhD, Vincent Jaquet, PhD, Karl-Heinz Krause, MD, Zaverio Ruggeri MD, Gabriela M. Kuster, MD, Jonathan R. Lindner, MD, Beat A. Kaufmann, MD

From the Department of Biomedicine and Division of Cardiology, University Hospital and University of Basel, Switzerland (E.K., M.M., L.X., M.M., G.M.K., B.A.K.), the Division of Cardiovascular Medicine, Oregon Health \& Science University(Y.Q., A.X., J.R.L.),the Department of Pathology and Immunology, Geneva Medical Faculty, Switzerland (V.J., K.H.K.), Clinical Pharmacology and Toxicology, Geneva University Hospital, Geneva, Switzerland (YD) and the Department of Molecular and Experimental Medicine; Scripps Research Institute, La Jolla, California (Z.M.R.).

Running title: Molecular imaging of anti-inflammatory effect of apocynin

Address correspondence to:

Beat A. Kaufmann, MD

Cardiology

University Hospital Basel

Petersgraben 4, 4031 Basel, Switzerland

Tel. +41612652525

Fax +41612654598

e-mail: kaufmannb@uhbs.ch

Keywords: Apocynin - Atherosclerosis - Oxidative Stress - Microbubbles Molecular Imaging

Subject codes:

Abbreviations: $\quad$ A.U. $=$ arbitrary units

$\mathrm{CEU}=$ contrast enhanced ultrasound

$\mathrm{HE}=$ hydroethidine

VCAM-1 = vascular cell adhesion molecule 1

Word count:

Total number of figures and tables: 5

TOC category: translational

TOC subcategory: Arteriosclerosis 
OBJECTIVE: Anti-oxidative drugs continue to be developed for the treatment of atherosclerosis. Apocynin is an NADPH-oxidase-inhibitor with anti-inflammatory properties. We used contrast enhanced ultrasound (CEU) molecular imaging to assess whether short-term apocynin therapy in atherosclerosis reduces vascular oxidative stress and endothelial activation

APPROACH AND RESULTS: Genetically-modified mice with early atherosclerosis were studied at baseline and after 7 days of therapy with apocynin $(4 \mathrm{mg} / \mathrm{kg} / \mathrm{d}$ I.P. $)$ or saline. CEU molecular imaging of the aorta was performed with microbubbles targeted to vascular cell adhesion molecule 1 (VCAM-1; MB $)$, to platelet GP1ba $\left(\mathrm{MB}_{\mathrm{Pl}}\right)$, and control microbubbles $\left(\mathrm{MB}_{\mathrm{Ctr}}\right)$. Aortic VCAM-1 was measured using Western Blot. Aortic ROS generation was measured using a lucigenin assay. Hydroethidine (HE) oxidation was used to assess aortic superoxide generation.

Baseline signal for $\mathrm{MB}_{\mathrm{V}}\left(1.3 \pm 0.3\right.$ A.U.) and $\mathrm{MB}_{\mathrm{Pl}}(1.5 \pm 0.5$ A.U.) was higher than for $\mathrm{MB}_{\mathrm{Ctr}}(0.5 \pm 0.2$ A.U., $\mathrm{p}<0.01)$. In saline-treated animals, signal did not significantly change for any microbubble agent whereas short-term apocynin significantly $(\mathrm{p}<0.05)$ reduced VCAM-1 and platelet signal $\left(\mathrm{MB}_{\mathrm{V}:} 0.3 \pm 0.1, \mathrm{MB}_{\mathrm{Pl}}: 0.4 \pm 0.1 \mathrm{MB}_{\mathrm{Ctr}}\right.$ : $0.3 \pm 0.2$ A.U., $p=0.6$ between agents). Apocynin reduced aortic VCAM-1 expression by $50 \%(\mathrm{p}<0.05)$. However, apocynin therapy did not reduce either ROS content, superoxide generation, or macrophage content..

CONCLUSIONS: Short-term treatment with apocynin in atherosclerosis reduces endothelial cell adhesion molecule expression. This change in endothelial phenotype can be detected by molecular imaging before any measurable decrease in macrophage content, and is not associated with a detectable change in oxidative burden. 


\section{INTRODUCTION}

Endothelial activation is a key step both in the initiation of atherosclerotic lesions as well as in their progression to a late stage, where inflammatory cell burden and susceptibility to acute atherothrombotic complications are high. Oxidative stress plays a major role in supporting and amplifying the endothelial activation in atherosclerosis $^{1}$. The family of NOX NADPH oxidase present in plaque macrophages, and in native endothelial and smooth muscle cells is a major source of reactive oxygen species (ROS) and therefore represents a potential therapeutic target 2.

Apocynin is a polyphenolic drug that has been isolated from plant extracts and inhibits assembly of the NOX2 isoform of the NADPH oxidase enzyme complex $^{3}$. In mice with advanced atherosclerosis, long-term therapy with apocynin has been shown to reduce endothelial adhesion molecule expression, platelet adhesion, and plaque growth; while in hypercholesterolemic rabbits apocynin started at a much earlier stage of disease has been shown to prevent development of atherosclerotic lesions ${ }^{4}$. It is unknown whether the beneficial effects of apocynin occur early after initiation of therapy. With regards to mechanism, it is unknown whether apocynin's effects are entirely due to a reduction in oxidative stress since polyphenolic drugs such as apocynin have anti-inflammatory effects independent of their anti-oxidant properties ${ }^{5,6}$. Direct anti-inflammatory action independent of antioxidant properties has been substantiated by the reduced adhesion molecule expression in cultured endothelial cells exposed to apocynin ${ }^{7,8}$. 
In this study we addressed many of these knowledge gaps. We performed in vivo ultrasound molecular imaging to test the hypothesis that short-term administration of apocynin in a model of early atherosclerosis reduces endothelial activation and platelet adhesion, two factors that are recognized to play an important role in plaque progression. Ex vivo techniques were used to evaluate whether these effects were associated with a reduction in vessel oxidative stress.

\section{METHODS}

\section{Mouse Model and Experimental Setup}

All experiments were performed in accordance with Swiss Federal Legislation and with the Guide for the Care and Use of Laboratory Animals of the National Institute of Health, and were approved by the local Animal Care and Use Committee at Oregon Health \& Science University and the Animal Care Committee of the Canton of Basel. Male mice with a double knockout for the LDL receptor and the Apobec-1 editing enzyme on a C57Bl/6 background were used. These mice develop atherosclerosis in a predictable, age-dependent fashion while on a normal chow diet. At 20 weeks of age, when the mouse model shows lesions that cover about $5 \%$ of the total aortic surface, and small fibrofatty lesions can be seen on histology ${ }^{9}$, the mice were treated with either apocynin $(4 \mathrm{mg} / \mathrm{kg} / \mathrm{d}$; acetovanillone, Sigma) $(\mathrm{n}=40)$ or $0.9 \%$ saline $(\mathrm{n}=40)$ daily by intraperitoneal route. Animals were studied after $7 \mathrm{~d}$ of therapy. A small subset of animals ( $\mathrm{n}=6$ for each treatment group) was also studied before initiation of therapy. For each imaging study aortic contrast-enhanced ultrasound for endothelial adhesion molecule expression and platelet adhesion was performed. Assessment of aortic oxidative stress, VCAM-1 expression and platelet adhesion were performed by a panel of histologic and tissue assay techniques. 


\section{Microbubble Preparation}

Biotinylated, lipid-shelled decafluorobutane microbubbles were prepared by sonication of a gas saturated aqueous suspension of distearoylphosphatidylcholine (2mg/ml; Avanti Polar Lipids, Alabaster AL), polyoxyethylene-40-stearate (1mg/ml; Sigma), and 1,2-distearoyl-sn-glycero-phosphoethanolamine-N[biotinyl(polyethylene glycol)-2000] (0.1mg/ml, Avanti Polar Lipids, Alabaster AL). Microbubbles targeted to VCAM-1 ( $\left.\mathrm{MB}_{\mathrm{VCAM}}\right)$ were prepared by conjugation of biotinylated rat anti-mouse VCAM-1 antibody (MK 2.7) to the microbubble surface using biotin-streptavidin-biotin linking as previously described ${ }^{10}$. Microbubbles targeted to GP1ba on activated platelets $\left(\mathrm{MB}_{\mathrm{Pl}}\right)$ were prepared by conjugating a biotinylated dimeric recombinant A1 domain (amino acids 445 to 909) of mouse von Willebrand factor (vWF) to the microbubbles. It has been shown previously that these microbubbles attach specifically to stationary platelet complexes even at high shear rates and that competitive inhibition from plasma vWF, or interaction with circulating platelets is minimal ${ }^{4}$. Control microbubbles $\left(\mathrm{MB}_{\mathrm{Ctr}}\right)$ bearing a nonspecific isotype control antibody (R3-34, BD Bioscience) were also prepared. Microbubble concentration and size were measured by electrozone sensing (Multisizer III, Beckman- Coulter). Microbubble mean size was not statistically different for the three microbubble preparations $\left(2.8 \pm 0.2\right.$ for $\mathrm{MB}_{\mathrm{Ctr}}, 2.8 \pm 0.2$ for $\mathrm{MB}_{\mathrm{VCAM}}, 2.9 \pm 0.2$ for $\left.\mathrm{MB}_{\mathrm{Pl}}\right)$. 


\section{Contrast Enhanced Ultrasound Molecular Imaging}

Ultrasound imaging (Sequoia Acuson C512; Siemens Medical Systems USA Inc., Mountain View, CA) was performed with a high-frequency linear-array probe (15L8) held in place by a railed gantry system. The ascending aorta including the sinus of valsalva of the mouse was imaged in a long axis plane from a right parasternal window. Contrast enhanced ultrasound (CEU) was performed with power modulation and pulse inversion (Contrast Pulse Sequence) imaging at a centerline frequency of $7 \mathrm{MHz}$ and a dynamic range of $50 \mathrm{~dB}$. The gain settings were adjusted to levels just below visible noise speckle and held constant. $\mathrm{MB}_{\mathrm{VCAM}}, \mathrm{MB}_{\mathrm{Pl}}$ or $\mathrm{MB}_{\mathrm{Ctr}}$ $\left(1 \times 10^{6}\right.$ microbubbles per injection) were injected intravenously in random order. Ultrasound imaging was paused from the time of injection until eight minutes later when imaging was resumed at a mechanical index of 0.87 . The first acquired image frame was used to derive the total amount of microbubbles present within the aorta. The microbubbles in the ultrasound beam were then destroyed with several $(>10)$ image frames. Several image frames at a long pulsing interval $(10 \mathrm{sec})$ were subsequently acquired to measure signal attributable to freely circulating microbubbles. Data were log-linear converted using known dynamic range lookup tables, and frames representing freely circulating microbubbles were digitally subtracted from the first image to derive signal from attached microbubbles alone. Contrast intensity was measured from a region of interest encompassing the sinus of valsalva, the ascending aorta and the initial portion of the aortic arch, extending into the origin of the brachiocephalic artery. The selection of the region of interest was guided by fundamental frequency anatomic images of the ascending aorta acquired at $14 \mathrm{MHz}$ at the end of each individual imaging sequence. 


\section{Echocardiography}

High frequency $(30 \mathrm{MHz})$ ultrasound imaging (Vevo 770, Visual Sonics Inc., Toronto, Canada) was performed for assessment of cardiac function. M-Mode images of the left ventricle at the height of the papillary muscles were used to calculate ejection fraction. ${ }^{\text {ref }}$ The aortic arch was imaged to measure internal diameter and the centerline aortic peak flow velocity in the same location was measured on pulsed-wave spectral doppler tracing as an index of aortic shear.

\section{Assessment of VCAM-1 and Plaque Macrophage Content}

VCAM-1 expression was assessed in four apocynin-treated and four saline-treated animals by Western blot and histology. For Western blot, the ascending portion of the aorta was homogenized in lysis buffer (Cell Signaling) containing $80 \mathrm{mmol} / \mathrm{L}$ Pefabloc SC plus (Roche). Protein concentration was measured using the Micro BCA (bicinchoninic acid) protein assay kit (Thermo Scientific). Ten microgram of protein were resolved on SDS-PAGE and transferred to Polyvinylidene fluoride (PVDF) membranes (Amersham). Membranes were probed with monoclonal rat anti-mouse VCAM-1 (Clone \# 112702, R\&D Systems) and monoclonal anti- $\alpha$-tubulin (Clone DM1A, Sigma) antibodies. Blots were subsequently incubated with horseradish peroxidase-conjugated secondary antibodies (Jackson Immuno Research) and band intensities were detected by enhanced chemiluminescence (Western Lightening Plus; Perkin Elmer) and quantitated using NIH ImageJ software (http://rsbweb.nih.gov/ij/).

Fluorescent immunohistochemistry was performed to spatially characterize the endothelial expression of VCAM-1 and to quantify plaque macrophage content. Frozen aortic sections were mounted on glass slides, fixed in $-20^{\circ} \mathrm{C}$ Acetone, air- 
dried, blocked with $10 \%$ goat serum in TBS/FSGO and incubated overnight at $4^{\circ} \mathrm{C}$ with monoclonal rat anti-VCAM-1 (CBL-1300, Millipore) and then for 1 hour at room temperature with goat anti-rat Alexa-633 (A21094, Invitrogen). Subsequently, Macrophages were labeled with a biotinylated rat anti Mac-2 (125403, Biolgegend). Fluorescent labeling of anti Mac-2 was accomplished with Alexa-594 labeled streptavidin (016-580-084, JacksonImmunoResearch). Sections were mounted with Prolong gold antifade mounting medium containing DAPI and imaged on a Zeiss LSM 710 confocal microscope. Alexa-633 representing VCAM-1 was detected with an excitation wavelength of $633 \mathrm{~nm}$ and an emission wavelength of $650-740 \mathrm{~nm}$ and Alexa594 representing Mac 2 with 594 excitation wavelength and 580-630 emission wavelength. Exposure time, averaging, and laser intensity were kept constant for all images. For spatial quantification of plaque macrophage content, the number of positively stained pixels was counted on threshold pictures and normalized to the total number of pixels of the plaque using Image-J. Thresholds were defined as the mean background intensity plus 40 times the standard deviation in each individual picture. For each mouse at least two cross-sections on different slides were imaged and quantified for both the base and ascending aorta.

\section{Assessment of endothelial platelet adhesion}

After the termination of imaging experiments, apocynin-treated and non-treated

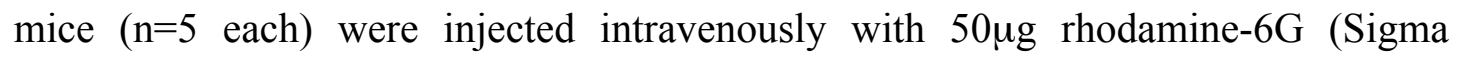
Aldrich). Ten minutes after injection, a right atrial incision was made through an anterior thoracotomy. The blood volume was removed with $10 \mathrm{ml}$ of $37^{\circ} \mathrm{C}$ phosphate buffered saline through a left ventricular puncture at an infusion pressure $\leq 100 \mathrm{~mm}$ 
Hg. The ascending aorta was then carefully removed, incised longitudinally, and pinned endothelial side facing up for en face fluorescent microscopy $(\times 20$ objective $)$ with epi-illumination at an excitation wavelength of $490 \mathrm{~nm}$. The degree of platelet/leukocyte complex attachment to the endothelium was quantified in 20 randomly selected non-overlapping visual fields by thresholding at $>10 \mathrm{SD}$ above normal endothelial surface with Image-J (National Institutes of Health, Bethesda, MD) and expressing area with positive fluorescence normalized to total endothelial surface area.

\section{NADPH oxidase activity and superoxide anion production}

For the assessment of NADPH oxidase activity, a right atrial incision was made through an anterior thoracotomy. The blood volume was removed with $10 \mathrm{ml}$ of $37^{\circ}$ C phosphate buffered saline through a left ventricular puncture at an infusion pressure $\leq 100 \mathrm{mmHg}$. The ascending aorta was carefully removed and cut into three circular segments of equal length, and the wet weight of the individual segments was measured. Aortic rings were incubated with $85 \mu \mathrm{L}$ of Jude Krebs Buffer $(119 \mathrm{mM}$ $\mathrm{NaCl}, 20 \mathrm{mM}$ HEPES, 4.6mM KCl, $1 \mathrm{mM} \mathrm{MgSO} 4,0.15 \mathrm{mM} \mathrm{Na}_{2} \mathrm{HPO}_{4} \cdot 2 \mathrm{H}_{2} \mathrm{O}, 0.4 \mathrm{mM}$ $\mathrm{KH}_{2} \mathrm{PO}_{4}, 5 \mathrm{mM} \mathrm{NaHCO}, 1.2 \mathrm{mM} \mathrm{CaCl}, 5.5 \mathrm{mM}$ Glucose) containing protease inhibitor (Roche) at $37^{\circ} \mathrm{C}$ for $30 \mathrm{~min}$. NOX activity was measured with a luminescence assay in a microplate luminometer with $2.5 \mu \mathrm{L}$ DMSO, $10 \mu \mathrm{M}$ lucigenin (Sigma) and $100 \mu \mathrm{M}$ NADPH (Sigma) per well (final volume 100uL/well). Data were recorded as relative light units over time, and integrated and calculated as area under the curve using Image J software for statistical analysis. 
For measurement of superoxide $\left(\mathrm{O}_{2}^{-}\right)$anion production, the aortas were removed as described above. The ascending aorta was dissected, cleaned from surrounding tissue and cut into rings of approximately 3 millimeters. The rings were incubated 30 minutes in $300 \mu \mathrm{l} \mathrm{HBSS}$ (Invitrogen) containing 50 $\mu \mathrm{M}$ Hydroethidine (HE) (Sigma Aldrich) at $37^{\circ} \mathrm{C}$ in the dark. The rings were then washed in phosphate buffered saline, snap frozen and kept at $-80^{\circ} \mathrm{C}$. The day of the experiment, the rings were homogenised in $370 \mu 1$ methanol. The homogenate was centrifuged at 13,000 rpm for 5 min and $50 \mu 1$ of supernatant was used for protein quantification using BIO-RAD protein assay (Bio-Rad Laboratories $\mathrm{GmbH}$, München) while the rest was dehydrated using a speed vacuum concentrator. The resulting pellet was dissolved in $100 \mu \mathrm{L} \mathrm{H}_{2} \mathrm{O}$ for HPLC analysis (1100 Series (Agilent, Palo Alto, Ca). Hydroethidine and its two oxidized products, i.e., superoxide-specific 2-hydroxyethidium and ethidium ${ }^{11}$ were separated by HPLC equipped with a fluorescence detector with excitation at $510 \mathrm{~nm}$ and emission at $595 \mathrm{~nm}$. The area under EOH peak was calculated and values were normalized to protein content of the rings.

\section{Statistical Analysis}

Data were analyzed on GraphPad Prism (version 5.0d). Data are expressed as mean \pm SEM unless stated otherwise. Single comparisons between the two animal groups were performed with a Mann-Whitney test. Kruskal-Wallis ANOVA with Dunn's post hoc test was used to compare microbubble signals within and between animal groups. For assessing the effect of treatment on targeted signals in the subgroup of animals that were imaged before and after treatment, a Wilcoxon 
matched-pairs signed rank test was used. A p value $<0.05$ (2-sided) was considered statistically significant.

\section{RESULTS}

\section{Effect of apocynin on VCAM-1 expression and endothelial platelet adhesion}

Apocynin treatment for 1 week reduced total aortic wall expression of VCAM-1 on Western blot by 50\% (Figure 1A). On immunohistochemistry, small regions of neointimal formation were observed in the aortic root and proximal ascending portion. VCAM-1 staining was present on the endothelial lining and on macrophages in fibrofatty lesions in non-treated animals. In apocynin-treated animals, VCAM-1 expression was reduced both on endothelial cells as well as on macrophages within lesions, while the total amount of macrophages present in plaques remained unchanged (Figure 2). The reduction in endothelial VCAM-1 expression was evident both in regions overlying plaques as well as on endothelium in regions without plaques (Figure 1B-E).

In vivo labeling of platelets with rhodamine-6G allowed visualization of platelet/leukocyte aggregates on the vascular endothelial surface. Platelet/leukocyte aggregates were present in regions with early plaques, but also in regions of the aortic endothelial surface with a normal appearance. Both the number of platelet/leukocyte aggregates per square millimeter $(0.33 \pm 0.065$ in apocynin treated animals vs $0.62 \pm 0.065$ in non-treated animals, $\mathrm{p}<0.01)$ and the percentage of endothelial surface covered by platelet/leukocyte aggregates was significantly reduced in animals that were treated with apocynin (Figure 3). 


\section{Effect of apocynin on vascular oxidative stress}

Lucigenin assays showed robust NADPH oxidase activity in whole aortic rings of non-treated mice. In mice treated with apocynin, NADPH-dependent lucigenin chemiluminescence was not different compared to non-treated mice. Given the potential of lucigenin to undergo redox cycling and generate artifactual signals, high pressure liquid chromatography (HPLC) of tissue extracts after exposure of aortic rings to $\mathrm{HE}$ was performed to directly assess tissue superoxide content. In accordance with the results of the lucigenin assays, DHE oxidation to EOH was not different between the two animal groups (Figure 4).

\section{Molecular Imaging of VCAM-1 expression and platelet adhesion}

High frequency ultrasound imaging was not of sufficient quality for evaluation in one animal in each group. In the remaining animals, there were differences in left ventricular ejection fraction, peak aortic flow velocity or aortic diameter, indicating that apocynin treatment did not lead to hemodynamic differences that could potentially influence targeted microbubble adhesion (table).

CEU molecular imaging in the ascending aorta at baseline showed greater signal enhancement for VCAM-1-targeted and platelet-targeted microbubbles compared to control microbubbles (Figure 5). After 7 days of treatment with apocynin, signal for VCAM-1 targeted and platelet targeted microbubbles was not different from control microbubble signal (Figure 5). In contrast, in animals treated with saline injections, the signal for VCAM-1 and platelets was elevated significantly over control signal to a degree that was similar to baseline. In the subgroup of animals that were imaged before and after treatment, apocynin lead to a significant decrease in VCAM-1 
targeted signal (from $1.80 \pm 0.51$ to $0.30 \pm 0.10, \mathrm{p}=0.046$ ) and a strong trend for decrease in platelet-targeted signal $(2.21 \pm 0.80$ to $0.35 \pm 0.07, \mathrm{p}=0.078)$. In animals

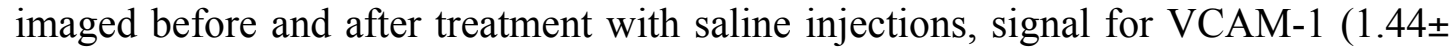
0.55 vs. $1.00 \pm 0.23, p=1.00)$ and platelet-targeted signal $(1.84 \pm 0.88$ vs. $1.26 \pm 0.40$ $\mathrm{p}=0.69)$ did not decrease significantly.

\section{DISCUSSION}

Endothelial activation plays a crucial role in the initiation and progression of atherosclerotic plaque formation. In this study, short term treatment with the polyphenol apocynin in a murine model of early mild atherosclerosis leads to a reduction in endothelial inflammatory phenotype and platelet adhesion. These relatively acute changes were not associated with a measurable reduction in vascular NADPH oxidase activity or superoxide content.

Endothelial adhesion molecule expression is an early and important step in the pathogenesis of atherosclerosis. Deposition of oxidized LDL in the vascular wall leads to endothelial expression of pro-inflammatory cytokines such as IL-1- $\beta$ and tumor necrosis factor- $\alpha$. Locally increased cytokine levels result in an upregulation of cell adhesion molecules such as VCAM-1, mediated by the transcriptional factors activated protein-1 (AP-1) and nuclear factor $\kappa \mathrm{B}(\mathrm{NF}-\mathrm{\kappa B})$, this in turn promotes the recruitment of leukocytes to the vessel wall.

ROS generated by NADPH oxidases both in endothelial cells as well as in leukocytes in nascent plaques are thought to amplify vascular inflammation throughout the pathogenesis of atherosclerosis. Accordingly, mouse models with 
knockouts of the NADPH oxidase isoforms NOX-1 and NOX-2 or the cytosolic NADPH oxidase subunit $\mathrm{p} 47 \mathrm{phox}$ have shown a reduction in atherosclerotic plaque formation ${ }^{12-14}$. In humans, functional deficiency of the GP91phox subunit is associated with smaller carotid intima-media thickness (CIMT) ${ }^{15}$. These findings have generated interest in using inhibitors of NADPH oxidases for the treatment of atherosclerosis. Apocynin inhibits NADPH oxidase activity in leukocytes allegedly by impeding the assembly of the cytosolic subunits at the cell membrane ${ }^{16}$. In cell culture experiments it has been noted that the inhibitory action of apocynin occurs with a delay, suggesting that it has to undergo activation before inhibiting ROS generation. In the presence of $\mathrm{H}_{2} \mathrm{O}_{2}$ and myeloperoxidase, apocynin is converted to an apocynin radical and subsequently forms apocynin dimers, which are thought to be the active compounds that result in inhibition of NADPH oxidase activity ${ }^{17}$. Thus, the effect of apocynin on NADPH oxidase inhibition during the later stages of atherosclerosis may depend on its activation in vascular tissue and require a sufficient inflammatory cell and oxidative stress burden. Notably, however, apocynin also exerts anti-inflammatory effects that are independent of NADPH oxidase inhibition ${ }^{6}$. Such effects may be of importance during the early stages of atherosclerosis, when inflammatory cell load and oxidative burden are low.

We examined the effects of treatment with apocynin in a murine model of early atherosclerosis in mice with fibrofatty lesions. Assessing treatment effects of potential drug candidates during the early stages of atherosclerosis is of clinical significance, since interventions that are started early during the pathogenesis of atherosclerosis are thought to afford a larger risk reduction for cardiovascular events than interventions that are initiated when clinical atherosclerotic disease is established 18. Our data indicate that treatment with apocynin results in a rapid decrease in 
endothelial expression of VCAM-1. These results are in line with observations in cell culture showing a decrease in VCAM-1 expression in response to apocynin ${ }^{8}$, and extend observations made in advanced atherosclerosis to earlier stages of plaque development ${ }^{4}$. In addition, the observation that the decrease in vascular inflammation was not associated with a reduction of vascular NADPH oxidase activity or tissue superoxide content indicates that the antiinflammatory effect of apocynin observed in our study was probably mediated through a ROS-independent mechanism in the very early stages of atherosclerotic plaque development, possibly mediated instead by its effects on cytochrome P450 pathways ${ }^{19}$.

In addition to endothelial cell inflammatory activation, platelet-endothelial interactions play a role in vascular inflammation and the pathogenesis of atherosclerosis. Platelet-endothelial interactions mediated by P-Selectin and von Willebrand factor-GPIb ligation in the absence of plaque rupture accelerate plaque formation in murine atherosclerosis ${ }^{20-22}$. The interaction of activated platelets with the endothelial surface results in the secretion of proinflammatory cytokines CD40L and IL-1 $\beta$, as well as of chemokines like RANTES (regulated on activation, normal T cell expressed and secreted) and platelet factor 4 (PF4) to the vascular wall, all of which facilitate increased monocyte recruitment ${ }^{23-25}$. Platelets from patients with functional deficiency of GP91phox subunit of NADPH oxidase or control subject platelets treated with apocynin have reduced in vitro platelet recruitment and aggregation, indicating a direct role of NADPH oxidase in platelet reactivity ${ }^{26}$. Our molecular imaging results indicate a decrease in platelet-endothelial interactions after treatment with apocynin. While we did not specifically investigate the pathways responsible for the action of apocynin in platelets, previous data indicate that apocynin influences platelet aggregation by mechanisms that are dependent on 
NADPH oxidase activity ${ }^{27}$ but also on changes in arachidonic acid metabolism with a decrease in thromboxane $\mathrm{A} 2$ formation ${ }^{5}$.

Several limitations of our study deserve attention. First, as the aim of our study was to assess the acute effects of apocynin treatment on the endothelial inflammatory phenotype, we did not expect an influence of treatment on plaque size and thus did not perform histological analysis. However, long-term treatment with apocynin has been shown to reduce plaque formation in our mouse model ${ }^{5}$. Also, the dose used in our study was in the low range of doses used in published animal studies, however, there is no established optimal dose, and our data demonstrate an effect of the treatment on both endothelial inflammatory phenotype and platelet adhesion. Furthermore, while we applied well-established techniques to measure NADPH oxidase activity and superoxide content without finding an effect of apocynin therapy, we cannot exclude that locally restricted (e.g. endothelial) and/or changes in other ROS species contributed to the observed effect. Finally, our methods for evaluating platelet adhesion did not allow differentiation of direct endothelial attachment and platelet-monocyte complexes.

In summary, we show that in a murine model of early atherosclerosis, treatment with apocynin leads to a rapid decrease in endothelial inflammation and platelet adhesion. Our data indicate that these effects of apocynin are not associated with a measurable decrease in ROS generation. 


\section{ACKNOWLEDGMENTS}

Sources of funding: This study was supported by SCORE grants from the Swiss National Science Foundation to Dr. Kaufmann (SNSF 32323B_123819 and 3232B0141603 ) and to Dr. Kuster (SNSF 3232B-111352 and 3200B-111353), as well as by grants R01-DK-063508, R01-HL-078610 and RC1-HL-100659; from the U.S. National Institutes of Health to Dr. Lindner. Dr. Khanicheh is supported by an MDPhD start up grant from the University Hospital Basel.

Disclosures: None.

\section{REFERENCES}

1. Singh U, Jialal I. Oxidative stress and atherosclerosis. Pathophysiology. 2006;13:129-142

2. Lassegue B, San Martin A, Griendling KK. Biochemistry, physiology, and pathophysiology of nadph oxidases in the cardiovascular system. Circ Res. 2012;110:1364-1390

3. Stolk J, Hiltermann TJ, Dijkman JH, Verhoeven AJ. Characteristics of the inhibition of nadph oxidase activation in neutrophils by apocynin, a methoxysubstituted catechol. Am J Respir Cell Mol Biol. 1994;11:95-102

4. Liu YN, Davidson BP, Yue Q, Belcik T, Xie A, Inaba Y, McCarty OJ, Tormoen GW, Zhao Y, Ruggeri ZM, Kaufmann BA, Lindner JR. Molecular imaging of inflammation and platelet adhesion in advanced atherosclerosis: 
Effects of antioxidant therapy with nadph oxidase inhibition. Circ Cardiovasc Imaging. 2012

5. Engels F, Renirie BF, Hart BA, Labadie RP, Nijkamp FP. Effects of apocynin, a drug isolated from the roots of picrorhiza kurroa, on arachidonic acid metabolism. FEBS Lett. 1992;305:254-256

6. Houser KR, Johnson DK, Ishmael FT. Anti-inflammatory effects of methoxyphenolic compounds on human airway cells. J Inflamm (Lond). 2012;9:6

7. Yu J, Weiwer M, Linhardt RJ, Dordick JS. The role of the methoxyphenol apocynin, a vascular nadph oxidase inhibitor, as a chemopreventative agent in the potential treatment of cardiovascular diseases. Curr Vasc Pharmacol. $2008 ; 6: 204-217$

8. Suzuki Y, Wang W, Vu TH, Raffin TA. Effect of nadph oxidase inhibition on endothelial cell elam-1 mrna expression. Biochem Biophys Res Commun. $1992 ; 184: 1339-1343$

9. Kaufmann BA, Carr CL, Belcik JT, Xie A, Yue Q, Chadderdon S, Caplan ES, Khangura J, Bullens S, Bunting S, Lindner JR. Molecular imaging of the initial inflammatory response in atherosclerosis: Implications for early detection of disease. Arterioscler Thromb Vasc Biol. 2010;30:54-59

10. Lindner JR, Song J, Christiansen J, Klibanov AL, Xu F, Ley K. Ultrasound assessment of inflammation and renal tissue injury with microbubbles targeted to p-selectin. Circulation. 2001;104:2107-2112 
11. Zhao H, Kalivendi S, Zhang H, Joseph J, Nithipatikom K, Vasquez-Vivar J, Kalyanaraman B. Superoxide reacts with hydroethidine but forms a fluorescent product that is distinctly different from ethidium: Potential implications in intracellular fluorescence detection of superoxide. Free Radic Biol Med. 2003;34:1359-1368

12. Sheehan AL, Carrell S, Johnson B, Stanic B, Banfi B, Miller FJ, Jr. Role for nox1 nadph oxidase in atherosclerosis. Atherosclerosis. 2011;216:321-326

13. Judkins CP, Diep H, Broughton BR, Mast AE, Hooker EU, Miller AA, Selemidis S, Dusting GJ, Sobey CG, Drummond GR. Direct evidence of a role for nox 2 in superoxide production, reduced nitric oxide bioavailability, and early atherosclerotic plaque formation in apoe-/- mice. Am J Physiol Heart Circ Physiol. 2010;298:H24-32

14. Vendrov AE, Hakim ZS, Madamanchi NR, Rojas M, Madamanchi C, Runge MS. Atherosclerosis is attenuated by limiting superoxide generation in both macrophages and vessel wall cells. Arterioscler Thromb Vasc Biol. $2007 ; 27: 2714-2721$

15. Violi F, Pignatelli P, Pignata C, Plebani A, Rossi P, Sanguigni V, Carnevale R, Soresina A, Finocchi A, Cirillo E, Catasca E, Angelico F, Loffredo L. Reduced atherosclerotic burden in subjects with genetically determined low oxidative stress. Arterioscler Thromb Vasc Biol. 2013;33:406-412

16. Barbieri SS, Cavalca V, Eligini S, Brambilla M, Caiani A, Tremoli E, Colli S. Apocynin prevents cyclooxygenase 2 expression in human monocytes through nadph oxidase and glutathione redox-dependent mechanisms. Free Radic Biol Med. 2004;37:156-165 
17. Johnson DK, Schillinger KJ, Kwait DM, Hughes CV, McNamara EJ, Ishmael F, O'Donnell RW, Chang MM, Hogg MG, Dordick JS, Santhanam L, Ziegler LM, Holland JA. Inhibition of nadph oxidase activation in endothelial cells by ortho-methoxy-substituted catechols. Endothelium. 2002;9:191-203

18. Ference BA, Yoo W, Alesh I, Mahajan N, Mirowska KK, Mewada A, Kahn J, Afonso L, Williams KA, Sr., Flack JM. Effect of long-term exposure to lower low-density lipoprotein cholesterol beginning early in life on the risk of coronary heart disease: A mendelian randomization analysis. $J$ Am Coll Cardiol. 2012;60:2631-2639

19. Pietersma A, de Jong N, de Wit LE, Kraak-Slee RG, Koster JF, Sluiter W. Evidence against the involvement of multiple radical generating sites in the expression of the vascular cell adhesion molecule-1. Free Radic Res. $1998 ; 28: 137-150$

20. Burger PC, Wagner DD. Platelet p-selectin facilitates atherosclerotic lesion development. Blood. 2003;101:2661-2666

21. Huo Y, Schober A, Forlow SB, Smith DF, Hyman MC, Jung S, Littman DR, Weber C, Ley K. Circulating activated platelets exacerbate atherosclerosis in mice deficient in apolipoprotein e. Nat Med. 2003;9:61-67

22. Massberg S, Brand K, Gruner S, Page S, Muller E, Muller I, Bergmeier W, Richter T, Lorenz M, Konrad I, Nieswandt B, Gawaz M. A critical role of platelet adhesion in the initiation of atherosclerotic lesion formation. $J \operatorname{Exp}$ Med. 2002;196:887-896 
23. Henn V, Slupsky JR, Grafe M, Anagnostopoulos I, Forster R, MullerBerghaus G, Kroczek RA. Cd40 ligand on activated platelets triggers an inflammatory reaction of endothelial cells. Nature. 1998;391:591-594

24. Hawrylowicz CM, Howells GL, Feldmann M. Platelet-derived interleukin 1 induces human endothelial adhesion molecule expression and cytokine production. J Exp Med. 1991;174:785-790

25. von Hundelshausen P, Weber KS, Huo Y, Proudfoot AE, Nelson PJ, Ley K, Weber C. Rantes deposition by platelets triggers monocyte arrest on inflamed and atherosclerotic endothelium. Circulation. 2001;103:1772-1777

26. Pignatelli P, Carnevale R, Di Santo S, Bartimoccia S, Sanguigni V, Lenti L, Finocchi A, Mendolicchio L, Soresina AR, Plebani A, Violi F. Inherited human gp91phox deficiency is associated with impaired isoprostane formation and platelet dysfunction. Arterioscler Thromb Vasc Biol. 2011;31:423-434

27. Begonja AJ, Gambaryan S, Geiger J, Aktas B, Pozgajova M, Nieswandt B, Walter U. Platelet nad(p)h-oxidase-generated ros production regulates alphaiibbeta3-integrin activation independent of the no/cgmp pathway. Blood. $2005 ; 106: 2757-2760$ 


\section{FIGURES}

Figure 1

A
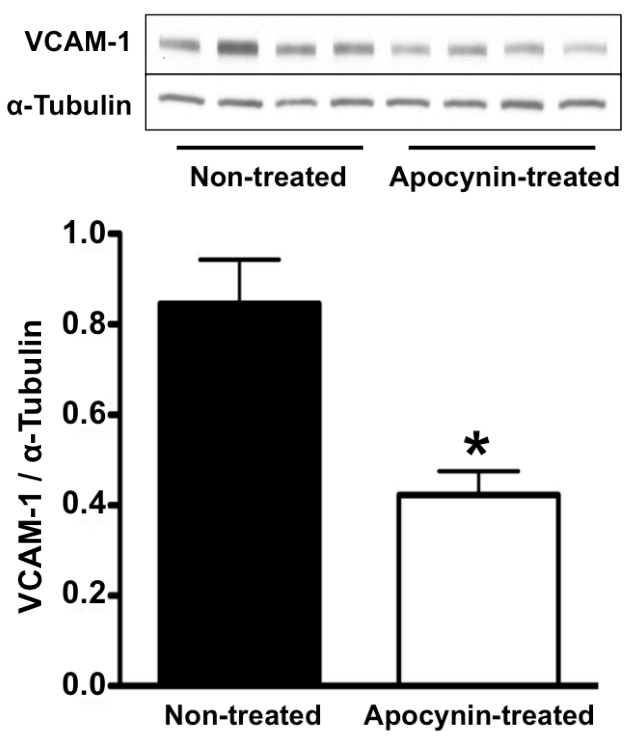
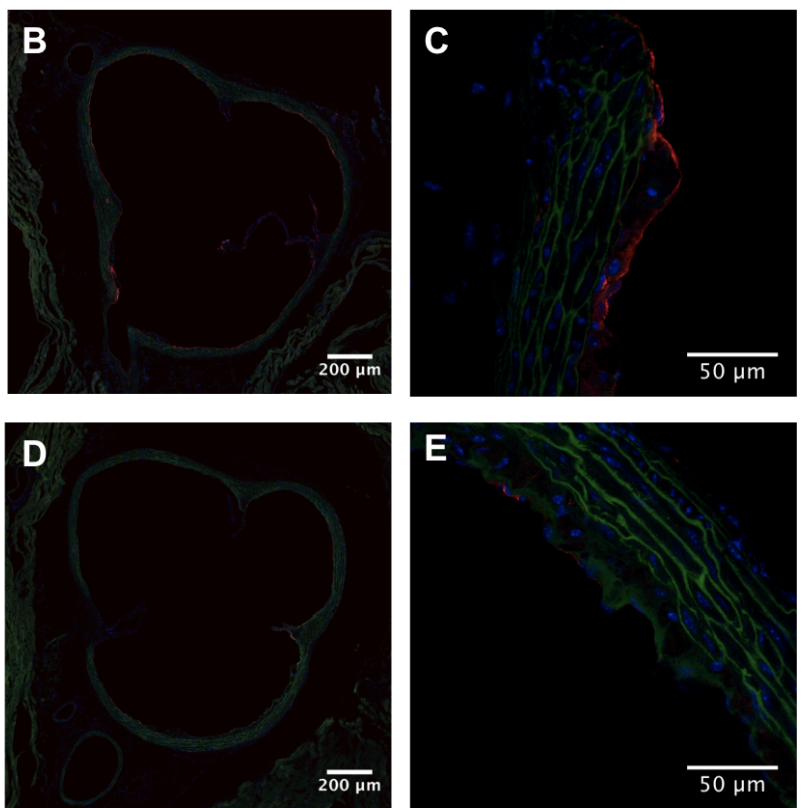

Figure 1. Assessment of VCAM-1 expression after 7 days of saline or apocynin treatment.

(A) VCAM-1 protein expression in the ascending aorta assessed by Western blot in non-treated (lanes 1-4) versus apocynin-treated (lanes 5-8) animals, $\mathrm{n}=4$ per group, ${ }^{*} \mathrm{p}<0.05$ vs non-treated animals. Representative examples of fluorescent immunohistochemistry images of the base of the aorta demonstrating endothelial VCAM-1 expression (red fluorescence) in a non-treated animal at 10-fold magnification (B), in the same animal at 40-fold magnification (C) and reduced VCAM-1 expression in an apocynin-treated treated animal (D, E). Autofluorescence delineating vessel anatomy is shown in green, DAPI staining of the nuclei in blue. 
Figure 2

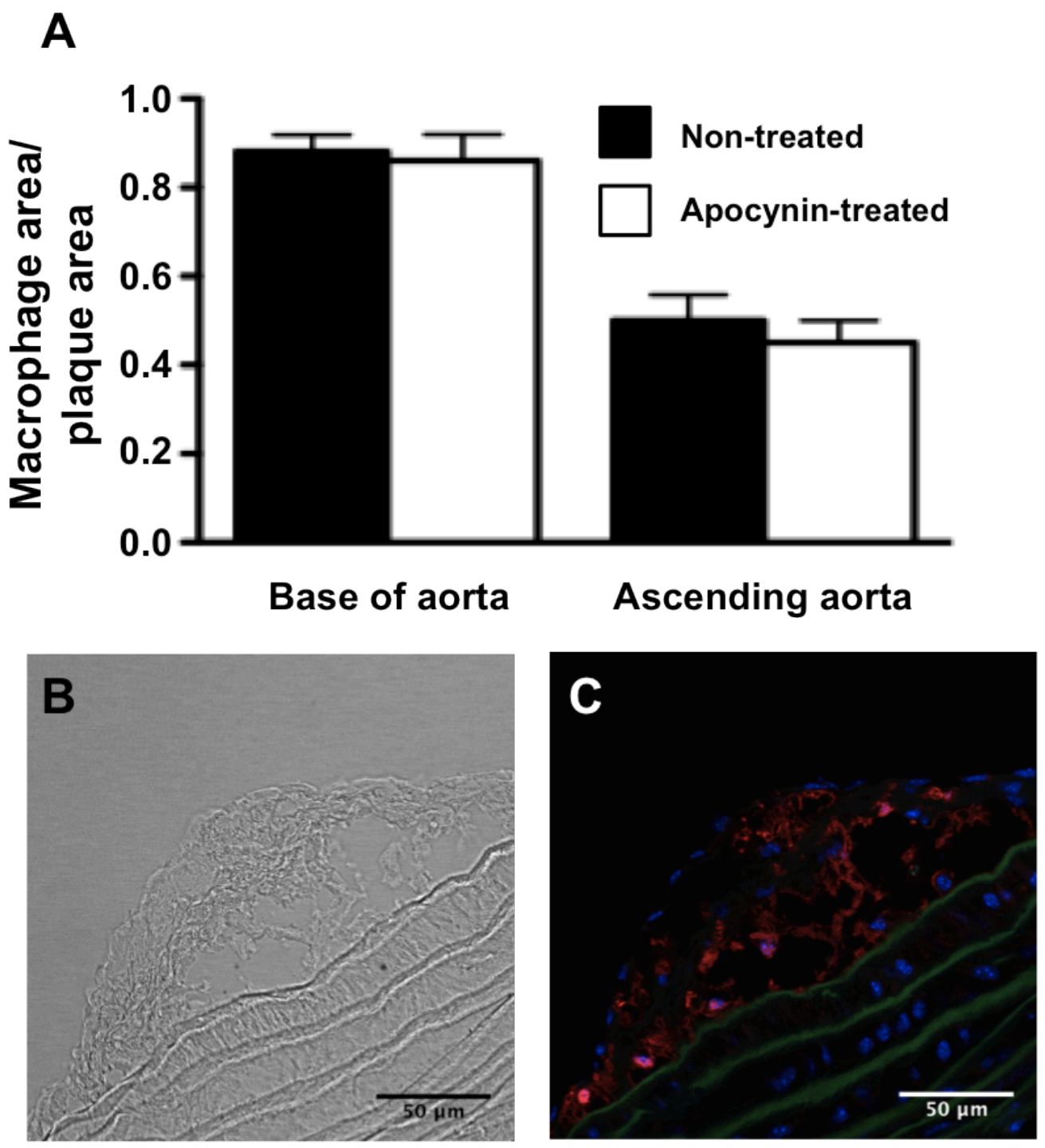

Figure 2. Assessment of plaque macrophage content after 7 days of saline or apocynin treatment.

(A) Percentage of the plaque area covered with macrophages (Mac-2 staining) at the base of the aorta and in the ascending aorta ( $\mathrm{n}=8$ in each group; $\mathrm{p}=$ ns vs non-treated). (B) Example of trans-illumination image used for plaque delineation. (C) Example of Mac-2 staining used for quantification of macrophage content in the plaque. 
Figure 3

A

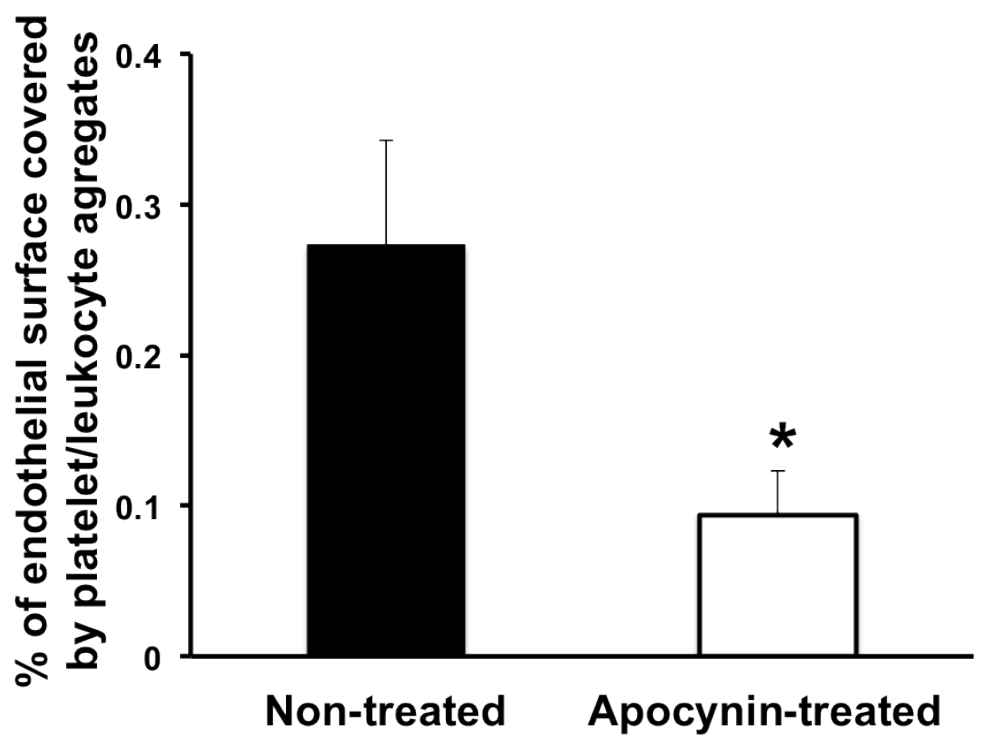

B
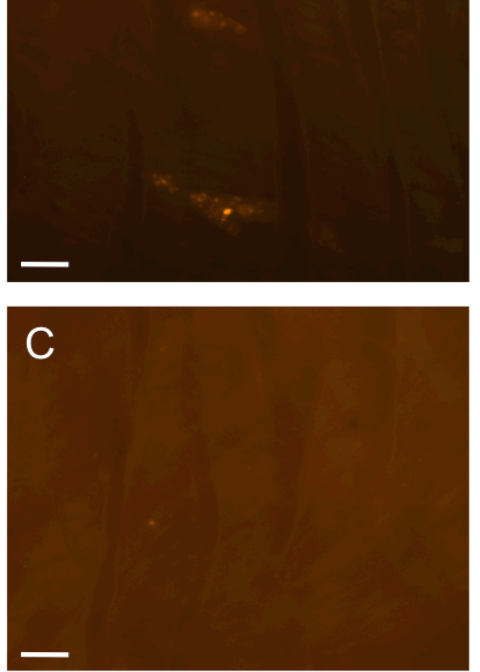

Figure 3. Assessment of platelet adhesion on the aortic endothelial surface after 7 days of saline or apocynin treatment.

(A) Percentage of the endothelial surface of the ascending aorta covered with platelet/leukocyte aggregates ( $\mathrm{n}=5$ in each group), ${ }^{*} \mathrm{p}<$ 0.05 vs non-treated animals. Examples of en face fluorescence microscopy demonstrating two platelet/leukocyte aggregates on normal appearing endothelial surface in a non-treated animal (B) and absence of platelet/leukocyte aggregates in an apocynin-treated animal (C). Scale bar $=25 \mu \mathrm{m}$ 
Figure 4
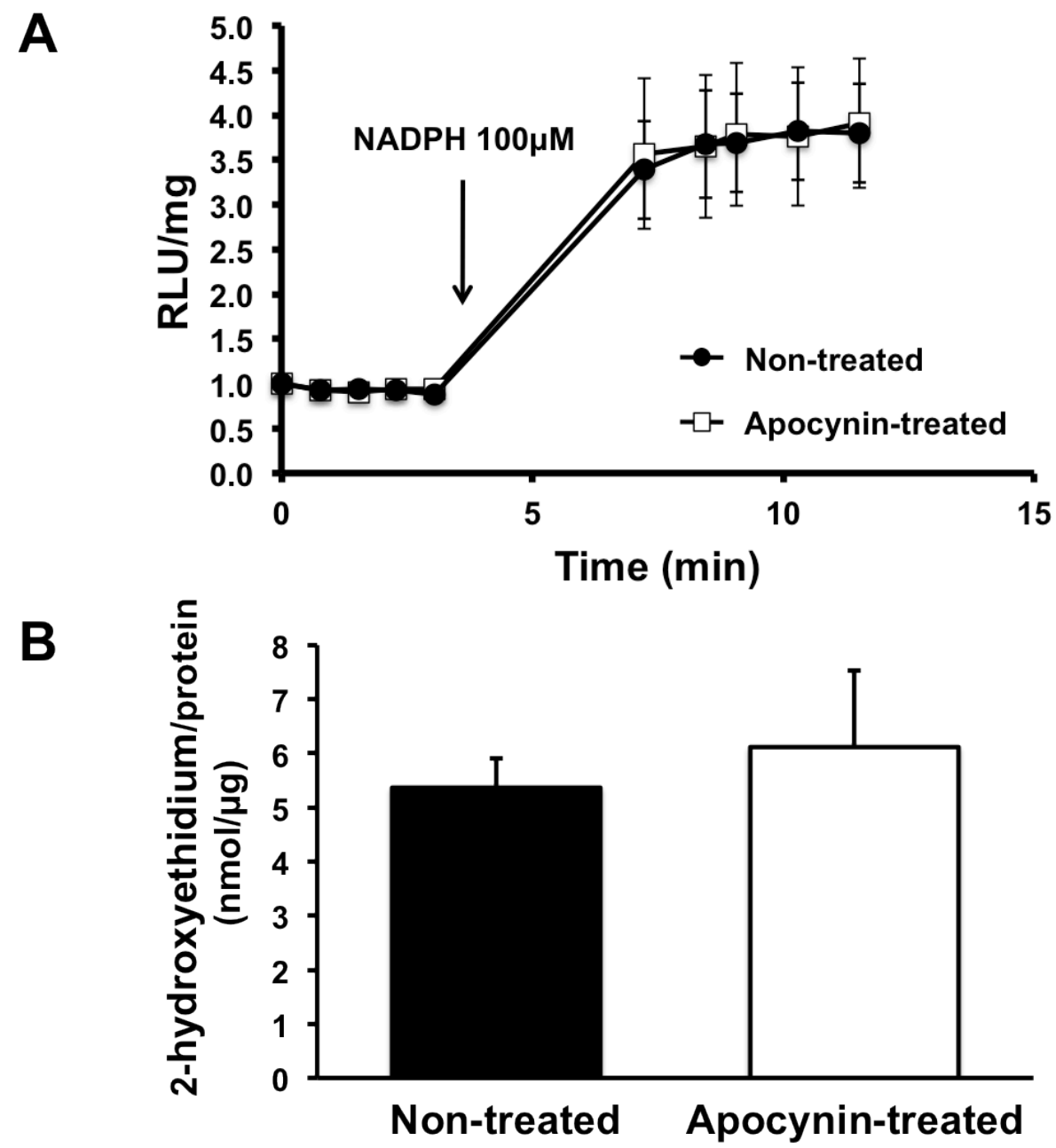

Figure 4. Assessment of ROS generation after 7 days of saline or apocynin treatment.

(A) Superoxide-generating activity of whole aortic rings after the addition of $100 \mu \mathrm{M}$ NADPH at 4 minutes. Measurements represent relative light units (RLU). $\mathrm{p}=\mathrm{ns}$ between saline-treated and apocynintreated animals (n=10 in each group). (B) HPLC analysis of EOH generated in vascular rings exposed to $50 \mu \mathrm{M}$ DHE. $\mathrm{p}=\mathrm{ns}$ between saline-treated and apocynin-treated animals ( $\mathrm{n}=8$ in each group). 
Figure 5

A
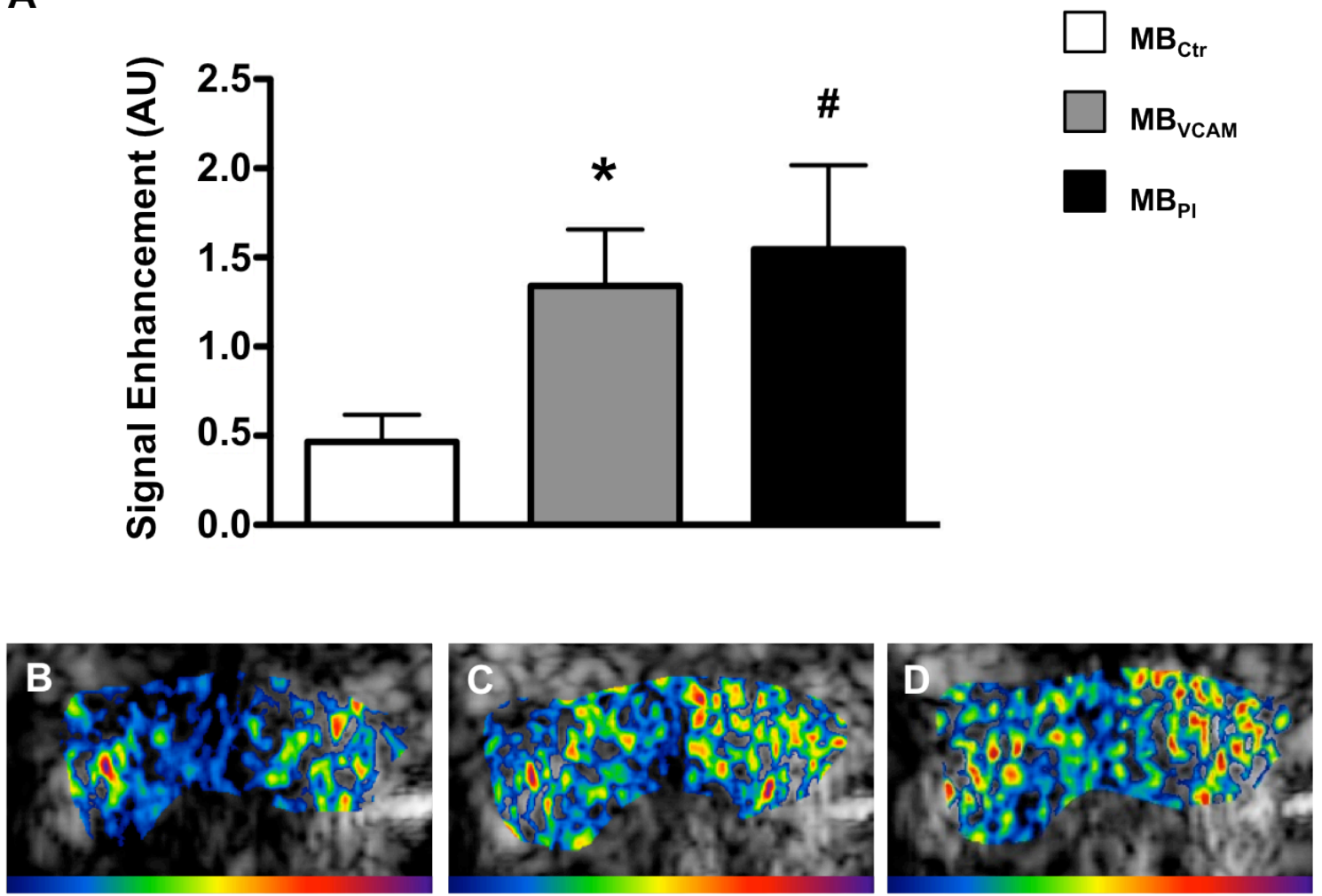

Figure 5. Molecular imaging of the ascending aorta before the start of treatment.

(A) Mean \pm SEM background-subtracted signal intensity for microbubbles targeted to VCAM-1 (MBVcam), to GP1b $\alpha$ on activated thrombocytes $\left(\mathrm{MBPl}_{\mathrm{Pl}}\right)$ and control microbubbles $\left(\mathrm{MB}_{\mathrm{ctr}}\right) .{ }^{*} \mathrm{p}<0.01$ vs $\mathrm{MB}_{\mathrm{ctr}}, \# \mathrm{p}<0.05$ vs $\mathrm{MB}_{\mathrm{ctr}}(\mathrm{n}=12)$. Examples of color coded CEU images after injection of $\mathrm{MB}_{\mathrm{Ctr}}(\mathbf{B})$, of $\mathrm{MB}_{\mathrm{VCAM}}$ (C) and of $\mathrm{MBPl}_{\mathrm{Pl}}$ (D). The color scale for the CEU images is shown at the bottom of each frame. 


\section{Figure 6}

A
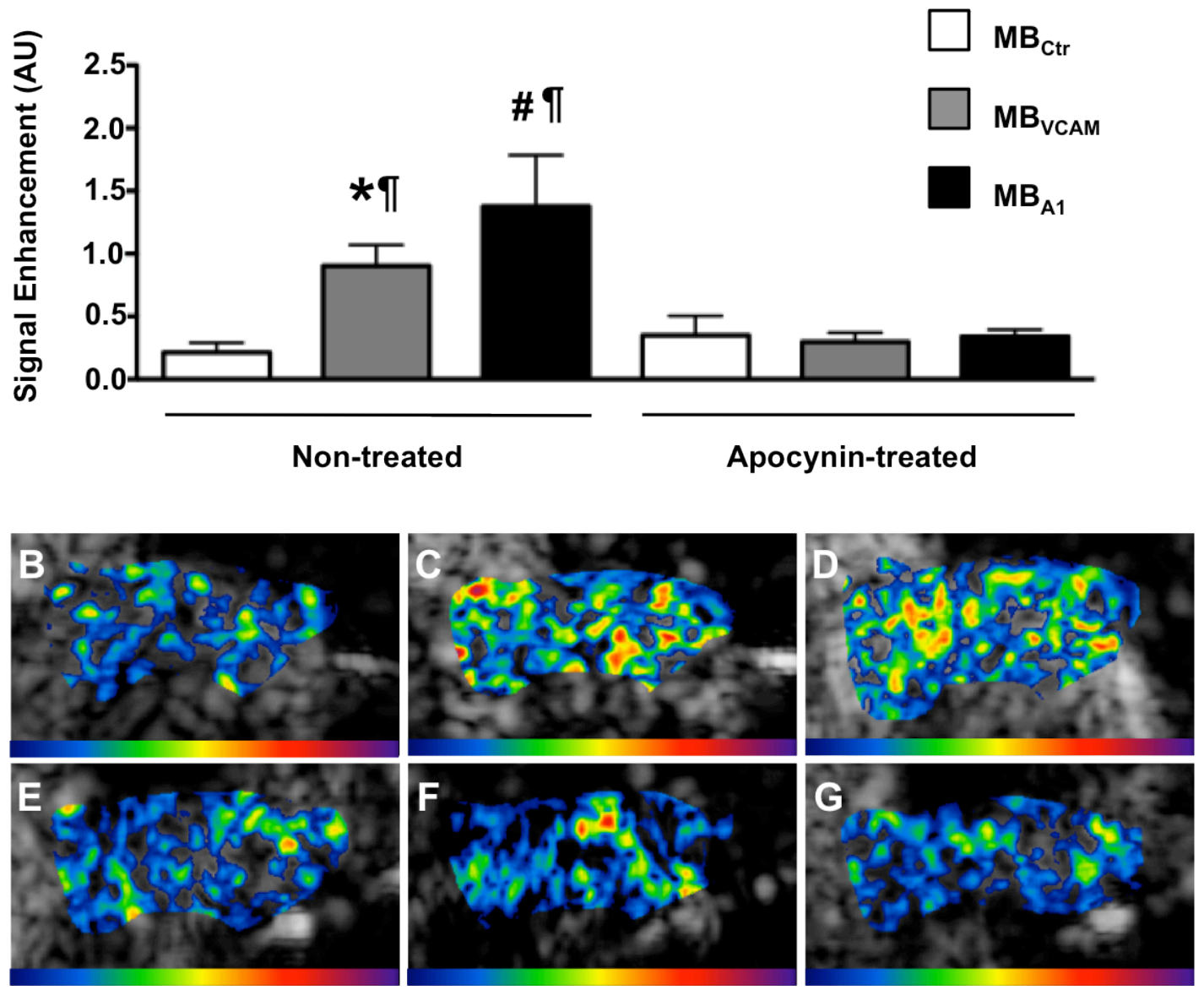

Figure 6. Molecular imaging of the ascending aorta after 7 days of saline or apocynin treatment.

(A) Mean \pm SEM background-subtracted signal intensity in salinetreated $(n=9)$ and apocynin-treated $(n=10)$ animals for microbubbles targeted to VCAM-1 (MB $\mathrm{VCAM}_{\mathrm{V}}$ ), to GP1b $\alpha$ on activated thrombocytes $\left(\mathrm{MB}_{\mathrm{Pl}}\right)$ and control microbubbles $\left(\mathrm{MB}_{\mathrm{Ctr}}\right) .{ }^{*} \mathrm{p}<0.01$ vs $\mathrm{MB}_{\mathrm{ctr}}, \# \mathrm{p}<0.01$ vs $\mathrm{MB}_{\text {ctr. }}$ If $\mathrm{p}<0.05$ vs the same microbubble in apocynin treated animals.

Examples of color coded CEU images after injection of $\mathrm{MB}_{\mathrm{Ctr}}$ (B), of $\mathrm{MB}_{\mathrm{VCAM}}$ (C) and of $\mathrm{MB}_{\mathrm{Pl}}$ (D) in saline treated animals. In the same 
order, examples of color coded CEU images after injection of $\mathrm{MB}_{\mathrm{Ctr}}$ (E), of $\mathrm{MBVCAM}_{\mathrm{V}}(\mathbf{F})$ and of $\mathrm{MB}_{\mathrm{Pl}}(\mathbf{G})$ in apocynin treated animals are shown.

Table. Echocardiographic data (mean \pm 1 SD)

\begin{tabular}{lccc}
\hline & $\begin{array}{c}\text { Saline- } \\
\text { treated } \\
(\mathbf{n}=\mathbf{8})\end{array}$ & $\begin{array}{c}\text { Apocynin- } \\
\text { treated } \\
(\mathbf{n}=9)\end{array}$ & P- value \\
\hline Ejection fraction (\%) & $58.4 \pm 13.1$ & $61.6 \pm 8.8$ & $\mathrm{~ns}$ \\
Aortic internal diameter (mm) & $1.54 \pm 0.09$ & $1.50 \pm 0.12$ & $\mathrm{~ns}$ \\
$\begin{array}{l}\text { Aortic peak systolic velocity } \\
(\mathbf{m} / \mathbf{s})\end{array}$ & $0.58 \pm 0.15$ & $0.68 \pm 0.03$ & $\mathrm{~ns}$ \\
\hline
\end{tabular}




\section{Discussion}

\subsection{The influence of the length of functionalized polyethylene glycol spacer arms on improving the targeting efficiency of microbubbles}

In recent years CEU molecular imaging has been used to non- invasively assess the pathophysiology of diseases at the molecular level. Lipid or albumin shell microbubbles with a heavy gas core are used as contrast agent. Imaging of the molecular footprints of the disease requires specific attachment of microbubbles to the molecule of interest. Therefore, for the specific targeting of microbubbles functionalized PEG spacer arms are also incorporated into the microbubble shell.

It has been shown that the targeting efficiency of microbubbles is low with retention of less than $5 \%$ of targeted microbubbles under high flow shear rate (54). In the present work we studied the influence of the length of PEG spacer arm on targeting efficiency of microbubbles. The improvement of targeting efficiency of microbubbles is also highly important where the targeted molecule is a relatively short molecule and is hidden in the glycocalyx.

Our study demonstrated that microbubbles with a longer PEG spacer arm yield a better targeting efficiency compared to the microbubbles with a shorter PEG spacer arm. In addition, reduced accessibility to the endothelial target due to the thickness of glycocalyx in the vessels may have an important influence on microbubble attachment.

The improvement of the targeting efficiency of microbubbles with longer PEG spacer arm could be due to better accessibility of the conjugated ligands on the microbubble surface to their endothelial target. In addition, bonds formed between ligands presented by longer PEG spacer arms to their target are more stable under flow condition. This can be explained by localizing the bond structure at the trailing point of the microbubble thus reducing torque forces exerted on the microbubbles under flow conditions (55). 
Interaction of targeted microbubbles with their endothelial target occurs in the presence of the endothelial glycocalyx. The glycocalyx could hinder ligandcounter ligand bond formations leading to lower signal obtained from microbubbles targeted to relatively short molecules hidden in the glycocalyx. Thus the low signal obtained when targeting the short cell adhesion molecule ICAM-1 could be secondary to glycocalyx steric hindrance or charge repulsion. However, the microbubbles with a longer PEG spacer arm still provided a higher ultrasound signal, possibly through increasing the chance of microbubble adhesion by projecting their ligands close enough to the endothelial surface for bond formation. In addition, degration of the glycocalyx resulted in an improvement of signal for microbubbles with different PEG spacer arm lengths.

\section{2 noninvasive ultrasound molecular imaging of the effect of statins on endothelial inflammatory phenotype in early murine atherosclerosis}

Atherosclerosis is a chronic inflammatory disorder and hence in recent years there has been a great interest in developing therapeutic regimes that target inflammatory pathways. Endothelial expression of the inflammatory molecule Vascular Cell Adhesion Molecule- 1 (VCAM-1) plays an important role in the initiation and progression of atherosclerosis. Improved strategies for atherosclerosis treatment require development of novel noninvasive imaging technologies that assess the effects of treatment on vascular inflammation. Statins have been used for the treatment of atherosclerosis. Their preventive effect is not only due to their potential in lowering plasma LDL cholesterol levels but also due to their anti- inflammatory effects. In the present study we investigated whether in vivo ultrasound molecular imaging of the expression of VCAM-1 could be used to assess the effects of statins on vascular endothelial phenotype in a mouse model of early atherosclerosis.

We could show that CEU molecular imaging can assess the impact of therapy on endothelial inflammation in a murine model of early atherosclerosis. In 
contrast high frequency ultrasound imaging of the aortic wall was unable to show effects of treatment on the atherosclerotic plaques.

It has been previously shown that targeted contrast based MRI and SPECT molecular imaging are cable of assessing the effects of statin treatment in animal models of advanced atherosclerosis. However, in the current study we provided evidence that CEU molecular imaging is capable of detecting changes in endothelial phenotype due to pharmacologic treatment in the very early stages of the atherosclerosis pathogenesis. An advantage of CEU molecular imaging is that it is capable of detecting targets specifically on the endothelial surface, whereas MRI and SPECT use diffusible tracers that need to accumulate in high concentrations both on the endothelial surface and within the plaque to be visualized. Therefore, given that phenotypic changes on the endothelial surface are responsible for the initiation of atherosclerosis, this method could be used not only to detect the initial phases of atherosclerosis pathogenesis but also for assessing treatment effects during the initial stages of atherosclerosis.

In our study the lower signal for microbubbles targeted to VCAM-1 on CEU molecular imaging of the ascending aorta in statin treated animals, was associated with a decrease in VCAM-1 expression and also a reduction in inflammatory cell content of atherosclerotic plaques. We also assessed aortic wall thickness with conventional high frequency ultrasound imaging. Although there was a difference of $40 \mu \mathrm{m}$ in aortic wall thickness on histology between the two groups of animals, high frequency ultrasound imaging with a theoretical axial resolution in the same range was unable to detect effects of treatment. Therefore at a time point where conventional ultrasound imaging was unable to detect early effects of treatment, non- invasive ultrasound molecular imaging of the aorta was able to detect effects of treatment on endothelial inflammation. 


\subsection{The short-term effects of treatment with apocynin on endothelial inflammation - application of ultrasound molecular imaging}

Endothelial activation is a key step both in initiation and progression of atherosclerosis. Oxidative stress plays an important role in supporting and intensifying endothelial activation. A major source of reactive oxygen species (ROS) is NADPH oxidase (NOX) in macrophages inside plaques as well as endothelial and smooth muscle cells. Apocynin is a polyphenolic drug that inhibits assembly of NOX complexes at the cell membrane. The beneficial effects of apocynin have been attributed to its anti-oxidative and antiinflammatory properties. However it is not yet clear whether these effects are present early in the development of atherosclerosis and with short term treatment. Thus in this study we used CEU molecular imaging to assess the short-term effects of apocynin on endothelial activation and investigated whether these anti-inflammatory effects are associated with reduced oxidative stress.

We could show that short-term treatment with apocynin in a mouse model of early atherosclerosis results in reduced aortic endothelial inflammation presented as a reduced expression of endothelial VCAM-1 and platelet adhesion. However, these anti inflammatory effects were not associated with measurable vascular NADPH oxidase activity or superoxide content.

These data are consistent with cell culture observations showing a decrease in endothelial VCAM-1 expression after exposure to apocynin and also in vivo studies after long-term treatment with apocynin in a mouse model of advanced atherosclerosis. However, the observed anti inflammatory response to apocynin in our study was not secondary to reduced NADPH oxidase activity or tissue ROS contents. The effects of apocynin are probably due to ROS- independent mechanisms in the early stages of atherosclerotic plaque development.

Adhesion of activated platelets to vascular endothelium can recruit monocytes into the vessel wall by producing pro-inflammatory cytokines thus, 
playing an important role in vascular inflammation and development of atherosclerosis. Our findings from CEU molecular imaging demonstrated that there is less attachment of activated platelets to aortic endothelial cells after treatment with apocynin. Although we did not investigate responsible pathways, previous studies indicate that apocynin influences platelet aggregation by different mechanisms involving NADPH oxidase activity and changes in arachidonic acid metabolism. 


\section{Abbreviations}

$\mathrm{Ab}$

Apo

Apobec

CEU

CT

$\mathrm{dB}$

$\mathrm{F}$

GP

HMG-CoA

ICAM

IL

IVUS

$\mathrm{kPa}$

LDL

MI

$\mathrm{MPa}$

MRI

mRNA

NADPH

P

PEG

PET

$\mathrm{RBC}$

ROS antibody

apolipoprotein

apolipoprotein B mRNA editing enzyme, catalytic polypeptide-like

contrast enhanced ultrasound

computed tomography

decibel

frequency

glycoprotein

hydroxymethylglutaryl- coenzyme A

intracellular adhesion molecule

interleukin

intravascular ultrasound

kilopascals

low density lipoprotein

mechanical index

megapascals

magnetic resonance imaging

messenger ribonucleic acid

nicotinamide adenine dinucleotide phosphate

pressure

polyethyleneglycol

positron emission tomography

red blood cell

reactive oxygen species 
TNF tumor necrosis factor

TF

tissue factor

TI

thermal index

VCAM

vascular cell adhesion molecule

vWF

von Willebrand factor 


\section{References}

1. Sinusas AJ, Bengel $F$, Nahrendorf $M$ et al. Multimodality cardiovascular molecular imaging, part I. Circulation Cardiovascular imaging 2008;1:244-56.

2. Inaba Y, Lindner JR. Molecular imaging of disease with targeted contrast ultrasound imaging. Translational research : the journal of laboratory and clinical medicine 2012;159:140-8.

3. Kaufmann BA, Lindner JR. Molecular imaging with targeted contrast ultrasound. Current opinion in biotechnology 2007;18:11-6.

4. Gramiak R, Shah PM. Echocardiography of the aortic root. Investigative radiology 1968;3:356-66.

5. Feinstein SB, Shah PM, Bing RJ et al. Microbubble dynamics visualized in the intact capillary circulation. J Am Coll Cardiol 1984;4:595-600.

6. H B. Handbook of Contrast Echocardiography LV Function and Myocardial Perfusion Berlin, Heiderberg, New York: Springer-Verlag, 2000.

7. Sirsi S, Borden M. Microbubble Compositions, Properties and Biomedical Applications. Bubble science engineering and technology 2009;1:3-17.

8. Du H, Chandaroy P, Hui SW. Grafted poly-(ethylene glycol) on lipid surfaces inhibits protein adsorption and cell adhesion. Biochimica et biophysica acta 1997;1326:236-48.

9. Efremova NV, Bondurant B, O'Brien DF, Leckband DE. Measurements of interbilayer forces and protein adsorption on uncharged lipid bilayers displaying poly(ethylene glycol) chains. Biochemistry 2000;39:3441-51.

10. Klibanov AL, Maruyama K, Torchilin VP, Huang L. Amphipathic polyethyleneglycols effectively prolong the circulation time of liposomes. FEBS letters 1990;268:235-7.

11. Klibanov AL, Maruyama K, Beckerleg AM, Torchilin VP, Huang L. Activity of amphipathic poly(ethylene glycol) 5000 to prolong the circulation time of liposomes depends on the liposome size and is unfavorable for immunoliposome binding to target. Biochimica et biophysica acta 1991;1062:142-8.

12. Torchilin VP, Omelyanenko VG, Papisov MI et al. Poly(ethylene glycol) on the liposome surface: on the mechanism of polymer-coated liposome longevity. Biochimica et biophysica acta 1994;1195:11-20.

13. Fisher NG, Christiansen JP, Klibanov A, Taylor RP, Kaul S, Lindner JR. Influence of microbubble surface charge on capillary transit and myocardial contrast enhancement. J Am Coll Cardiol 2002;40:811-9.

14. Sklenar J, Jayaweera AR, Kaul S. A computer-aided approach for the quantitation of regional left ventricular function using two-dimensional echocardiography. J Am Soc Echocardiogr 1992;5:33-40.

15. Masugata H, Peters B, Lafitte S, Strachan GM, Ohmori K, DeMaria AN. Quantitative assessment of myocardial perfusion during graded 
coronary stenosis by real-time myocardial contrast echo refilling curves. J Am Coll Cardiol 2001;37:262-9.

16. Jayaweera AR, Edwards N, Glasheen WP, Villanueva FS, Abbott RD, Kaul S. In vivo myocardial kinetics of air-filled albumin microbubbles during myocardial contrast echocardiography. Comparison with radiolabeled red blood cells. Circ Res 1994;74:1157-65.

17. Lindner JR, Song J, Jayaweera AR, Sklenar J, Kaul S. Microvascular rheology of Definity microbubbles after intra-arterial and intravenous administration. J Am Soc Echocardiogr 2002;15:396-403.

18. Villanueva FS, Jankowski RJ, Klibanov $S$ et al. Microbubbles targeted to intercellular adhesion molecule-1 bind to activated coronary artery endothelial cells. Circulation 1998;98:1-5.

19. Kaufmann BA, Sanders JM, Davis $C$ et al. Molecular imaging of inflammation in atherosclerosis with targeted ultrasound detection of vascular cell adhesion molecule-1. Circulation 2007;116:276-84.

20. Leong-Poi H, Christiansen J, Klibanov AL, Kaul S, Lindner JR. Noninvasive assessment of angiogenesis by ultrasound and microbubbles targeted to alpha(v)-integrins. Circulation 2003;107:45560.

21. Hamilton AJ, Huang SL, Warnick D et al. Intravascular ultrasound molecular imaging of atheroma components in vivo. J Am Coll Cardiol 2004;43:453-60.

22. Lanza GM, Wallace KD, Scott MJ et al. A novel site-targeted ultrasonic contrast agent with broad biomedical application. Circulation 1996;94:3334-40.

23. Schumann PA, Christiansen JP, Quigley RM et al. Targetedmicrobubble binding selectively to GPIIb Illa receptors of platelet thrombi. Investigative radiology 2002;37:587-93.

24. Lanza GM, Abendschein DR, Hall CS et al. Molecular imaging of stretch-induced tissue factor expression in carotid arteries with intravascular ultrasound. Investigative radiology 2000;35:227-34.

25. Lindner JR, Coggins MP, Kaul S, Klibanov AL, Brandenburger GH, Ley $\mathrm{K}$. Microbubble persistence in the microcirculation during ischemia/reperfusion and inflammation is caused by integrin- and complement-mediated adherence to activated leukocytes. Circulation 2000;101:668-75.

26. Tsutsui JM, Xie F, Cano $\mathrm{M}$ et al. Detection of retained microbubbles in carotid arteries with real-time low mechanical index imaging in the setting of endothelial dysfunction. J Am Coll Cardiol 2004;44:1036-46.

27. Lindner JR, Dayton PA, Coggins MP et al. Noninvasive imaging of inflammation by ultrasound detection of phagocytosed microbubbles. Circulation 2000;102:531-8.

28. Lindner JR, Song J, Xu F et al. Noninvasive ultrasound imaging of inflammation using microbubbles targeted to activated leukocytes. Circulation 2000;102:2745-50.

29. Lanza GM, Wickline SA. Targeted ultrasonic contrast agents for molecular imaging and therapy. Curr Probl Cardiol 2003;28:625-53.

30. Piedra M, Allroggen A, Lindner JR. Molecular imaging with targeted contrast ultrasound. Cerebrovasc Dis 2009;27 Suppl 2:66-74.

31. Kaseda N, Uehara Y, Yamamoto Y, Tanaka K. Induction of in situ immune complexes in rat glomeruli using avidin, a native cation 
macromolecule. British journal of experimental pathology 1985;66:72935.

32. Lindner JR, Song J, Christiansen J, Klibanov AL, Xu F, Ley K. Ultrasound assessment of inflammation and renal tissue injury with microbubbles targeted to P-selectin. Circulation 2001;104:2107-12.

33. Behm CZ, Kaufmann BA, Carr $\mathrm{C}$ et al. Molecular imaging of endothelial vascular cell adhesion molecule-1 expression and inflammatory cell recruitment during vasculogenesis and ischemiamediated arteriogenesis. Circulation 2008;117:2902-11.

34. Kaufmann BA, Lewis C, Xie A, Mirza-Mohd A, Lindner JR. Detection of recent myocardial ischaemia by molecular imaging of $\mathrm{P}$-selectin with targeted contrast echocardiography. European heart journal 2007;28:2011-7.

35. Eriksson EE, Werr J, Guo Y, Thoren P, Lindbom L. Direct observations in vivo on the role of endothelial selectins and alpha(4) integrin in cytokine-induced leukocyte-endothelium interactions in the mouse aorta. Circ Res 2000;86:526-33.

36. Greve JM, Les AS, Tang BT et al. Allometric scaling of wall shear stress from mice to humans: quantification using cine phase-contrast $\mathrm{MRI}$ and computational fluid dynamics. American journal of physiology Heart and circulatory physiology 2006;291:H1700-8.

37. Takalkar AM, Klibanov AL, Rychak JJ, Lindner JR, Ley K. Binding and detachment dynamics of microbubbles targeted to P-selectin under controlled shear flow. Journal of controlled release : official journal of the Controlled Release Society 2004;96:473-82.

38. Kaufmann BA. Ultrasound molecular imaging of atherosclerosis. Cardiovascular research 2009;83:617-25.

39. Dayton PA, Chomas JE, Lum AF et al. Optical and acoustical dynamics of microbubble contrast agents inside neutrophils. Biophysical journal 2001;80:1547-56.

40. Lankford M, Behm CZ, Yeh J, Klibanov AL, Robinson P, Lindner JR. Effect of microbubble ligation to cells on ultrasound signal enhancement: implications for targeted imaging. Investigative radiology 2006;41:721-8.

41. Dayton PA, Pearson D, Clark J et al. Ultrasonic analysis of peptideand antibody-targeted microbubble contrast agents for molecular imaging of alphavbeta3-expressing cells. Molecular imaging 2004;3:125-34.

42. McCarty OJ, Conley RB, Shentu W et al. Molecular imaging of activated von Willebrand factor to detect high-risk atherosclerotic phenotype. JACC Cardiovascular imaging 2010;3:947-55.

43. Yang J, Hirata $\mathrm{T}$, Croce $\mathrm{K}$ et al. Targeted gene disruption demonstrates that P-selectin glycoprotein ligand 1 (PSGL-1) is required for P-selectin-mediated but not E-selectin-mediated neutrophil rolling and migration. The Journal of experimental medicine 1999;190:1769-82.

44. Dansky HM, Barlow CB, Lominska C et al. Adhesion of monocytes to arterial endothelium and initiation of atherosclerosis are critically dependent on vascular cell adhesion molecule-1 gene dosage. Arteriosclerosis, thrombosis, and vascular biology 2001;21:1662-7. 
45. Walpola PL, Gotlieb Al, Cybulsky MI, Langille BL. Expression of ICAM1 and VCAM-1 and monocyte adherence in arteries exposed to altered shear stress. Arteriosclerosis, thrombosis, and vascular biology 1995;15:2-10.

46. Weller GE, Villanueva FS, Klibanov AL, Wagner WR. Modulating targeted adhesion of an ultrasound contrast agent to dysfunctional endothelium. Annals of biomedical engineering 2002;30:1012-9.

47. Kaufmann BA, Carr CL, Belcik JT et al. Molecular imaging of the initial inflammatory response in atherosclerosis: implications for early detection of disease. Arteriosclerosis, thrombosis, and vascular biology 2010;30:54-9.

48. van Diepen JA, Berbee JF, Havekes LM, Rensen PC. Interactions between inflammation and lipid metabolism: Relevance for efficacy of anti-inflammatory drugs in the treatment of atherosclerosis. Atherosclerosis 2013.

49. Bustos $\mathrm{C}$, Hernandez-Presa MA, Ortego $\mathrm{M}$ et al. HMG-CoA reductase inhibition by atorvastatin reduces neointimal inflammation in a rabbit model of atherosclerosis. J Am Coll Cardiol 1998;32:2057-64.

50. Sukhova GK, Williams JK, Libby P. Statins reduce inflammation in atheroma of nonhuman primates independent of effects on serum cholesterol. Arteriosclerosis, thrombosis, and vascular biology 2002;22:1452-8.

51. Liao JK. Rosuvastatin to prevent vascular events in men and women with elevated C-reactive protein. Current atherosclerosis reports 2009;11:243-4.

52. Pedersen TR, Faergeman O, Kastelein JJ et al. High-dose atorvastatin vs usual-dose simvastatin for secondary prevention after myocardial infarction: the IDEAL study: a randomized controlled trial. JAMA : the journal of the American Medical Association 2005;294:2437-45.

53. Li L, Chu Y, Fink GD, Engelhardt JF, Heistad DD, Chen AF. Endothelin-1 stimulates arterial VCAM-1 expression via NADPH oxidase-derived superoxide in mineralocorticoid hypertension. Hypertension 2003;42:997-1003.

54. Guenther F, von zur Muhlen C, Ferrante EA, Grundmann S, Bode C, Klibanov AL. An ultrasound contrast agent targeted to P-selectin detects activated platelets at supra-arterial shear flow conditions. Investigative radiology 2010;45:586-91.

55. Ham AS, Klibanov AL, Lawrence MB. Action at a distance: lengthening adhesion bonds with poly(ethylene glycol) spacers enhances mechanically stressed affinity for improved vascular targeting of microparticles. Langmuir : the ACS journal of surfaces and colloids 2009;25:10038-44. 
7. Curriculum vitae 


\section{Curriculum vitae}

Dr. Elham Khanicheh MD, MSc

Departement Biomedizin

Labor 303

Universitätsspital Basel

Hebelstrasse 20

4031 Basel

Email: Elham.khanicheh@unibas.ch

\section{Universitäre und klinische Ausbildung}

Mai 2010-Mai 2013 Doktorat in Medizinisch-biologischer Forschung (MD$\mathrm{PhD}$ ), Universitätsspital Basel, Schweiz, Doktorarbeit: Cardiovascular Molecular Imaging. Supervisor: PD Dr. Beat A. Kaufmann

$2009-2010$ Master of Global Health, Karolinska Institut, Stockholm, Schweden

2007-2009

Hausärtzin an der Bahar Andishe Poliklinik, Teheran Iran

2005-2007

Ärztin Allgemeinmedizin: Ashkanan Beesat Spital, Lamerd, Iran

$1998-2005$

Medizinstudium und Doktorat der Medizin (MD), Shahid Beheshti Universität, Tehran, Iran

\section{Diplome, Zertifikate, Mitgliedschaften}

2012

2010

2005

2005
ECFMG (USA- Educational Commission for Foreign Medical Graduate) Zertifikat

Master of Global Health, Karolinska Institute, Schweden, Masterarbeit: Effect of climate change on children health. Supervisors: Dr. md Elisabet Lingren, Dr. md Tobias Alfven

Registrierung bei der Iranischen Ärztekammer

Doktor der Medizin, Shahid Beheshti Universität, Iran, Doktorarbeit: Study of carbon monoxide poisoning in children referring to loqman Hakim hospital. abgeschlossen 2005, Supervisor: Dr. md Azar Falah, Note:3.8/4 


\section{Auszeichnungen}

2011

Finalistin am Investigators Award European Society of Echocardiography, Budapest, Ungarn (1 von 3 Finalisten)

2008 USMLE (United States Medical Licensing Exam) Step II: 240, 99. Perzentile

USMLE Step I: 219, 91. Perzentile

Grant

$2011 / 2012$

MD-PhD Grant des Universitätsspitals Basel

\section{Publikationen (Science Journal und Präsentationen)}

\section{a) Publikationen (peer reviewed)}

1. Khanicheh E, Mitterhuber M, Xu L,Häuselmann SP,Kuster GM,Kaufmann BA. Noninvasive ultrasound molecular imaging of the effects of statin on endothelial inflammatory phenotype in early atherosclerosis. PLoS ONE 8(3): e58761, 2013.

2. Khanicheh E, Mitterhuber M, Kinslechner K, Xu L, Lindner JR, Kaufmann BA. Factors affecting the endothelial retention of targeted microbubbles, Influence of microbubble shell design and cell surface projection of the endothelial target molecule. J Am Soc Echocardiogr. 2012 Apr; 25(4): 460-6.

3. Yegane RA, Alaee MS, Khanicheh E. Congenital plexiform Schwannoma of the clitoris: report of a case. Saudi Med J. 2008, Apr; 29(4):600-2.

\section{b) Publikationen (abstracts)}

Khanicheh E, Qi Y, Xie A, Mitterhuber M, Xu L, Mochizuki M, Daali Y, Jaquet V, Krause KH, Ruggeri Z, Kuster GM, Lindner JR, Kaufmann BA. Rapid reduction of endothelial activation in early-stage atherosclerosis with apocynin independent of anti- oxidative properties. AGLA \& Cardiovascular Biology, Poster Präsentation, Bern, Schweiz, 2013.

Guenther F, Kaufmann BA, Klibanov AL, Heidt T, Kramer M, Khanicheh E, Ferrante EA, Neudorfer I, Yasuhiro K, Peter K, Bode C, von zur Muhlen C. Dual microbubble targeting improves in vitro capture efficiency of ultrasound microbubbles targeted to selectins and activated platelets although not providing additional signal effect for in 
vivo imaging of arterial thrombosis. American Heart Association, Los Angles, USA, 2012, abstract Akzeptiert.

Khanicheh E, Mitterhuber M, Xu L,Häuselmann SP,Kuster GM,Kaufmann BA. Noninvasive ultrasound molecular imaging of the effect of atorvastatin on vascular inflammation. European Society of Echocardiography, Mündlichen Vortrag, Budapest, Ungarn, 2011.

Khanicheh E, Mitterhuber M, Kinslechner K, Xu L, Lindner JR, Kaufmann BA. Influence of microbubble shell design on attachment efficiency in targeted ultrasound molecular imaging. Schweizerische Gesellschaft für Kardiologie, Mündlichen Vortrag, Basel, Schweiz, 2011.

\section{Sprachen}

Farsi:

Englisch:

Deutsch:
Muttersprache

Sehr gut in Wort und Schrift

Gut in Wort und Schrift 